\title{
PRODUCTS AND COMMUTATORS OF CLASSES IN ALGEBRAIC GROUPS
}

\author{
ROBERT GURALNICK AND GUNTER MALLE
}

Dedicated to the memory of Tonny Springer

\begin{abstract}
We classify pairs of conjugacy classes in almost simple algebraic groups whose product consists of finitely many classes. This leads to several interesting families of examples which are related to a generalization of the Baer-Suzuki theorem for finite groups. We also answer a question of Pavel Shumyatsky on commutators of pairs of conjugacy classes in simple algebraic groups. It turns out that the resulting examples are exactly those for which the product also consists of only finitely many classes.
\end{abstract}

\section{INTRODUCTION}

The Baer-Suzuki theorem asserts that in a finite group $G$, if $x \in G$ is such that $\left\langle x, x^{g}\right\rangle$ is a $p$-group for all $g \in G$, then $\left\langle x^{G}\right\rangle$ is a normal $p$-subgroup of $G$.

We were recently informed by Bernd Fischer that Reinhold Baer had asked what one can say if, given $x, y \in G$, we have that $\left\langle x, y^{g}\right\rangle$ is a $p$-group for all $g \in G$. Examples in Guralnick-Malle-Tiep [7] show that there is not too much to say in general. However, with some extra hypothesis [7, 6], there are generalizations along these lines.

Here we consider the corresponding question for almost simple algebraic groups. Our main result is the following characterization, part of which is an analogue of the corresponding result in the connected case [7, Cor. 5.14]:

Theorem 1.1. Let $G$ be an almost simple algebraic group over an algebraically closed field, and let $C, D$ be $G^{\circ}$-classes. The following are equivalent:

(i) $C D$ is a finite union of $G^{\circ}$-conjugacy classes.

(ii) $C_{G^{\circ}}\left(x_{1}\right) \backslash G^{\circ} / C_{G^{\circ}}\left(x_{2}\right)$ is finite for all $\left(x_{1}, x_{2}\right) \in C \times D$.

(iii) $\left\langle x_{1}, x_{2}\right\rangle$ normalizes some Borel subgroup of $G^{\circ}$ for every $\left(x_{1}, x_{2}\right) \in C \times D$.

Moreover, any of the above conditions implies:

(iv) $[C, D]:=\{[x, y] \mid(x, y) \in C \times D\}$ is a finite union of $G^{\circ}$-conjugacy classes.

Here, an almost simple algebraic group is a possibly disconnected linear algebraic group whose connected component $G^{\circ}$ is simple and such that $C_{G}\left(G^{\circ}\right)=Z\left(G^{\circ}\right)$. See Theorem 6.4 for further equivalent conditions. Note that the result holds for $G$-classes as well

Date: October 31, 2018.

2010 Mathematics Subject Classification. Primary 20G07; Secondary 20E32, 20E45, 20F12.

Key words and phrases. conjugacy classes, commutators, algebraic groups.

The first author was partially supported by the NSF grant DMS-1001962 and the Simons Foundation Fellowship 224965. The second author gratefully acknowledges financial support by ERC Advanced Grant 291512. 
as long as we avoid the case $G=D_{4} \cdot \mathfrak{S}_{3}$ (because in the disconnected cases one of the classes is outer and so the centralizer intersects all cosets except in the excluded case).

While some implications in this statement have general proofs, others rely on our explicit classification of all pairs of classes with the stated properties.

Thus, we first classify in Theorem 3.7 all pairs $C, D$ of unipotent classes in (disconnected) almost simple algebraic groups such that every pair in $C \times D$ generates a unipotent group (the connected case was done in [7]). In fact, it turns out to be equivalent to the condition that $C D$ consists of unipotent elements.

We also extend the result of [7, Thm. 1.1] to the disconnected case by classifying pairs $C, D$ of conjugacy classes in (disconnected) almost simple groups such that $C D$ is a finite union of conjugacy classes.

It should be noted that for connected groups examples occur if and only if the group is of non-simply laced type, while for disconnected groups examples exist if and (clearly) only if the type is simply laced. See Proposition 4.12 for some relation between these two types of examples. Let's point out the following consequence of our classification: whenever $C D$ is a finite union of conjugacy classes, then at least one of $C, D$ contains a quasi-central class in its closure (recall that a quasi-central class is the smallest conjugacy in a given coset of $G^{\circ}$ ). It would be nice to have an a priori proof of this.

Two corollaries of the results and proofs are (extending the results of [7] to the disconnected case):

Corollary 1.2. Let $G$ be an almost simple algebraic group over an algebraically closed field. Suppose that $C$ and $D$ are conjugacy classes of $G$. Then either $C D$ is the union of at most 3 conjugacy classes or $C D$ contains infinitely many conjugacy classes.

In the previous result, if we replace $G$-classes by $G^{\circ}$-classes, then 5 classes suffice.

Corollary 1.3. Let $G$ be an almost simple algebraic group over an algebraically closed field $k$. If $x \in G \backslash Z\left(G^{\circ}\right)$, then $\operatorname{dim}\left(C_{G}(x) y C_{G}(x)\right)<\operatorname{dim} G$ for all $y \in G$, unless $G=D_{4}(k) . \mathfrak{S}_{3}$ and $x$ is an outer involution with $C_{G}(x)=B_{3}(k)$ and $y$ is not in $\left\langle G^{\circ}, x\right\rangle$.

Note that in the excluded case, we really get counter-examples. This last corollary in the connected case was used by Prasad 12 in his study of quasi-reductive groups.

We also answer in the case of algebraic groups a question that Pavel Shumyatsky had asked in the finite case:

Theorem 1.4. Let $G$ be an almost simple algebraic group over an algebraically closed field of characteristic $p \geq 0$. Let $C$ be $a G^{\circ}$-conjugacy class of $G$ outside $Z\left(G^{\circ}\right)$. Then $[C, C]$ is the union of infinitely many conjugacy classes, where as before,

$$
[C, C]:=\{[x, y] \mid x, y \in C\} .
$$

It is worth noting that we also observe that an old result of the first author [4] answers a question of Nick Katz [8, 2.16.7] about commutators being transvections. This is useful in the proof of the algebraic group result.

Finally, we observe that it is quite easy to extend our results to almost simple algebraic groups over infinite fields. In particular, we can answer the following question of Diaconis. 
Theorem 1.5. Let $G$ be a simple compact Lie group. If $C$ and $D$ are noncentral conjugacy classes of $G$, then $C D$ consists of infinitely many conjugacy classes.

The paper is organized as follows. In Section 2 we collect some auxiliary results on closed conjugacy classes in not necessarily connected algebraic groups. We then consider products of unipotent classes in disconnected algebraic groups in Section 3 and classify all cases where the product consists of finitely many classes in Theorem 3.7. In Section 4 we classify arbitrary products of classes meeting only finitely many conjugacy classes and thus prove Theorem 4.6. In Section 5 we show that these are precisely the pairs of conjugacy classes whose commutator consists just of finitely many classes, see Theorem 5.2. In Section 6, we prove the common characterization in Theorem 1.1 of various finiteness properties on products, commutators and double cosets and prove Corollary [1.2. In Section 17, we fully investigate the products of two non-trivial cosets of order 2 in the disconnected groups of type $D_{4}(k) \cdot \mathfrak{S}_{3}$. In the final section, we point out how we may extend our results to the case of infinite fields.

The similar questions for finite groups are considerably harder and will be dealt with in a forthcoming paper [6].

We thank Pavel Shumyatsky for communicating his question to us, George McNinch for pointing out the relevance of the results of Richardson [13], and Sebastian Herpel for some comments on a preliminary version.

\section{Closed CONJugacy Classes in Disconnected Algebraic Groups}

We'll need some information on closed conjugacy classes. In connected reductive algebraic groups, the closed classes are precisely the semisimple ones. In the disconnected case, there can be non-semisimple elements whose class is closed. Following Steinberg [17, $\S 9]$ we call an automorphism of a connected reductive algebraic group quasi-semisimple if it normalizes a Borel subgroup and a maximal torus thereof. We also use the following result of Spaltenstein [15, II.2.21]:

Theorem 2.1 (Spaltenstein). Let $G$ be a reductive algebraic group over an algebraically closed field. Then any coset of $G^{\circ}$ in $G$ contains at most one closed unipotent class. In particular, this class is contained in the closure of all unipotent classes in that coset.

Lemma 2.2. Let $H$ be a simple algebraic group over an algebraically closed field of characteristic $p>0$. Assume that $u$ is a quasi-semisimple automorphism of $H$ of order $p$ and $s \in H$ is a semisimple element commuting with $u$. Then us is also quasi-semisimple.

Proof. By assumption $u$ normalizes a Borel subgroup $B$ of $H$ and a maximal torus $T \leq B$. Let $D:=C_{H}(u)$. By [2, Thm. 1.8(iii)], $T \cap D$ is a maximal torus of $D, B \cap D$ is a Borel subgroup of $D$ and $D$ is connected (see also Steinberg [17, 8.2] for the simply connected case). (Alternatively, this can be seen by inspection of the various cases.) Thus, $s$ is conjugate in $D$ to an element of $T \cap D$ and so we may assume that $s \in T \cap D$. Then us normalizes $T$ and $B$.

Theorem 2.3. Let $G$ be a reductive algebraic group over an algebraically closed field $k$ of characteristic $p$ and let $C \subset G$ be a conjugacy class, $g \in C$. Then the following are equivalent: 
(i) $C$ is closed;

(ii) $C_{G}(g)$ is reductive; and

(iii) $g$ is quasi-semisimple.

Proof. The equivalence of (i) and (iii) is shown in [15, Lemme II.1.15] (see also [15, Cor. II.2.22]).

Now assume (i), so $C$ is closed. Then $C$ is an affine variety (since $G$ is affine). It follows by [13, Lemma 10.1.3] that $C_{G}(g)$ is reductive.

Finally let's show that (ii) implies (iii). Note that all the conclusions concern only $\left\langle g, G^{\circ}\right\rangle$ and so we assume that $G=\left\langle g, G^{\circ}\right\rangle$. There is no loss in assuming that $G^{\circ}$ is semisimple with $C_{G}\left(G^{\circ}\right)=1$. Moreover, we may assume that $g$ acts transitively on the simple components of $G^{\circ}$. If there are $m$ components, then $C_{G^{\circ}}(g) \cong C_{L}\left(g^{m}\right)$ where the components are isomorphic to $L$. Moreover, $g$ is quasi-semisimple if and only if $g^{m}$ is. Thus, it suffices to assume that $G^{\circ}$ is simple.

Assume that $g$ is not quasi-semisimple. We show that $C_{G}(g)$ is not reductive.

Write $g=s u=u s$ where $s$ is semisimple and $u$ is unipotent. Let $v$ be a generator of $\langle u\rangle \cap G^{\circ}$. If $v \neq 1$, then by the Borel-Tits theorem, the unipotent radical $U$ of $C_{G^{\circ}}(s v)$ is nontrivial, whence since $u$ acts as a $p$-element on $U, C_{U}(u)^{\circ}$ is a positive dimensional subgroup contained in the unipotent radical of $C_{G}(g)$. So $v=1$, and hence $u^{p}=1$.

If $p \neq\left[G: G^{\circ}\right]$, then $g=s$ is semisimple and in particular quasi-semisimple. Thus we may assume that $p=\left[G: G^{\circ}\right]$ (in particular, $p=2$ or 3 ).

By the discussion above, we have that $u$ is an automorphism of order $p$ and $s \in G^{\circ}$. Note that $u$ does not lie in the unique class of outer unipotent elements which are quasisemisimple, since otherwise by the previous lemma, $g$ would also be quasi-semisimple.

We consider the various cases. For the moment, exclude the case that $p=2$ and $G^{\circ}=D_{n}(k), n \geq 4$. If $p=2$, it follows by [1, 19.7-19.9] that $u=v t$ where $v$ is a quasi-semisimple graph automorphism and $t$ is a long root element in $C_{G^{\circ}}(v)$. But then, $C_{G^{\circ}}(u)<C_{G^{\circ}}(v)$ has a nontrivial unipotent radical. The same argument applies for $p=3$ and $G=D_{4}(k)$ [3, 4.9.2].

Finally, consider the case that $G^{\circ}=D_{n}(k), n \geq 4$ and $p=2$. Let $V$ be the natural $2 n$-dimensional orthogonal module for $G$.

Suppose that $s=1$. Note that $u$ is not a transvection on $V$ (which is the unique quasisemisimple unipotent class in $u G^{\circ}$ ). Thus, the fixed space $W$ of $u$ has dimension at most $2 n-2$. Let $R$ be the radical of $W$ (with respect to the alternating form preserved by $G$ ). Note that $R \neq 0$ for otherwise, $W$ is nonsingular and so $V=W \oplus W^{\perp}$, a contradiction since $u$ will have fixed points on $W^{\perp}$. If $R$ is totally singular with respect to the quadratic form preserved by $G$, then the centralizer of $u$ is contained in the parabolic subgroup stabilizing $R$ and so $C_{Q}(u)$ is a positive dimensional subgroup of the unipotent radical of $C_{G^{\circ}}(u)$, which thus is not reductive. Indeed the same argument applies if $\operatorname{dim} R>1$, for then replace $R$ by $R^{\prime}$, the radical with respect to the quadratic form. So $R$ is 1-dimensional and is generated by a non-isotropic vector. The stabilizer of this 1-dimensional space is $\langle x\rangle \times \operatorname{Sp}_{2 n-2}(k)$ with $x$ a transvection. If $u \notin\langle x\rangle$, then $C_{G^{\circ}}(u) \cong C_{\operatorname{Sp}_{2 n-2}(k)}(y)$ for some nontrivial unipotent element $y$ and so is not reductive.

If $s \neq 1$, write $V=W \perp W^{\prime}$ where $W=[s, V]$. Note that $W \neq 0$ since $s \neq 1$. Also, (since $p=2$ ) the centralizer of $s$ in the stabilizer of $W$ is contained in $\operatorname{SO}(W)$. In particular, if $u$ acts nontrivially on $W$, it follows (by inspection or Borel-Tits) that the 
centralizer of $G$ has non-trivial unipotent radical on $W$ and so on $V$. Thus, $u$ is trivial on $W$. Since the centralizer of $u$ on $W^{\prime}$ is reductive, it follows by the previous case that $u$ induces a transvection on $W^{\prime}$, whence $u$ is a transvection. This contradiction completes the proof.

Note that the proof actually also shows that:

Corollary 2.4. Let $G$ be a reductive algebraic group over an algebraically closed field. Let $g \in G$ with semisimple part s. There exists a unique closed conjugacy class in $g G^{\circ}$ whose elements have semisimple part conjugate to $s$.

We also have the obvious corollary.

Corollary 2.5. Let $G$ be a reductive algebraic group over an algebraically closed field $k$. Assume that the order of $g$ in $G / G^{\circ}$ is prime to the characteristic of $k$. Then the class of $g$ is closed if and only if $g$ is semisimple.

Proof. In this situation, quasi-semisimple elements are semisimple (see [17, §9]), and the result follows.

\section{Pairs of unipotent Classes in almost simple Algebraic Groups}

In this section we classify pairs of unipotent conjugacy classes in almost simple algebraic groups such that all pairs of elements generate a unipotent subgroup (see Theorem 3.7). Note that the connected case was already treated in [7, Thm. 1.1]. First note the following:

Lemma 3.1. Let $G$ be a reductive algebraic group and $C_{1}, C_{2}, D \subset G$ three $G^{\circ}$-orbits under conjugation. Assume that $D \subseteq C_{1} C_{2}$ and $\operatorname{dim} C_{1}+\operatorname{dim} C_{2}=\operatorname{dim} D$. Then:

(a) $D$ is open dense in the product $C_{1} C_{2}$;

(b) $\left\{\left(c_{1}, c_{2}\right) \in C_{1} \times C_{2} \mid c_{1} c_{2} \in D\right\}$ is a dense open subset of $C_{1} \times C_{2}$;

(c) $C_{1} C_{2}$ consists of finitely many classes.

Proof. Since $G^{\circ}$ is connected, the $G^{\circ}$-orbits $C_{1}, C_{2}, D$ are irreducible and then so is $C_{1} C_{2}$. Now $\operatorname{dim} C_{1} C_{2} \leq \operatorname{dim} C_{1}+\operatorname{dim} C_{2}=\operatorname{dim} D$ and $D \subset C_{1} C_{2}$ imply that $\operatorname{dim} C_{1} C_{2}=\operatorname{dim} D$. Thus, their closures agree. Since any two dense subsets intersect non-trivially, $D$ is the unique dense class in $C_{1} C_{2}$, so it must be open. The second statement now follows. As the closure of any class consists of finitely many classes, we have (c).

We now collect some actual examples:

Example 3.2. Let $S=\mathrm{SL}_{2 n}(k), n \geq 2$, with $k$ an algebraically closed field. Let $x \in S$ be a transvection, $y \in \operatorname{Aut}(S)$ a graph automorphism with centralizer $\operatorname{Sp}_{2 n}(k)$ and $G=\langle S, y\rangle$. Let $U$ be a root subgroup containing $x$. Let $N=N_{S}(U)$. Since $\operatorname{Sp}_{2 n}(k)$ has two orbits on pairs consisting of a 1-space and a hyperplane containing it we see that $\left|\operatorname{Sp}_{2 n}(k) \backslash S / N\right|=2$. Thus, $x^{S} \times y^{S}$ consists of two $S$-orbits. One orbit is the set of commuting pairs. When $k$ has characteristic $p>0$, reducing to the case of $n=2$ shows that in the other orbit the product will have order $2 p$. In particular, if $p=2,\left\langle x, y^{g}\right\rangle$ is either elementary abelian of order 4 or a dihedral group of order 8 for all $g \in S$. 
Example 3.3. Let $G=\mathrm{GO}_{2 n}(k), n \geq 4$, with $k$ an algebraically closed field of characteristic 2. Then $G$ fixes a non-degenerate alternating form $($,$) on V=k^{2 n}$, so we may view $G$ as a subgroup of the isometry group $\operatorname{Sp}_{2 n}(k)$ of this form. Let $x \in G$ be a transvection, and $y$ an involution with $(y v, v)=0$ for all $v \in V$. Then by [7, Ex. 6.6], the order of $x y$ is either 2 or 4 , so $x, y$ always generate a 2 -group.

Example 3.4. Let $k$ be an algebraically closed field of characteristic 3 and $G=\mathrm{SO}_{8}(k) .3$, the extension of the simple algebraic group $G^{\circ}=\mathrm{SO}_{8}(k)$ by a graph automorphism of order 3. Let $C_{1}$ be the class of long root elements in $G^{\circ}$, with centralizer of type $3 A_{1}$, and $C_{2}$ the class of the graph automorphism $\sigma$ with centralizer $G_{2}(k)$ in $G^{\circ}$. We claim that $C_{1} C_{2}$ consists of 3 -elements.

Indeed, let $x_{i}(1), 1 \leq i \leq 4$, denote long root elements for the four simple roots, labelled so that the node in the centre of the Dynkin diagram has label 2, and $\sigma$ permutes $x_{1}(1), x_{3}(1), x_{4}(1)$ cyclically. So $x:=x_{2}(1) \in C_{1}$, and it's easily seen that $y:=$ $x_{1}(1) x_{3}(1) x_{4}(1) \sigma$ is conjugate to $\sigma$, so lies in $C_{2}$. The product $x y=x_{2}(1) x_{1}(1) x_{3}(1) x_{4}(1) \sigma$ lies in a class $D$, denoted $C_{4}$ in [15, I.3], of dimension 24 (see also the representative $u_{4}$ in [10, Tab. 8]). Now $C_{1}$ has dimension 10 and $C_{2}$ has dimension 14. Since $C_{1}$ is a single class under $G^{\circ}$-conjugation, and $C_{2}, D$, being outer classes, necessarily are, Lemma 3.1 shows that $D$ is dense in $C_{1} C_{2}$.

Since $\sigma$ normalizes the maximal unipotent subgroup generated by the standard positive root subgroups, $\langle x, y\rangle$ is unipotent for our choice of elements $x \in C_{1}, y \in C_{2}$ above, hence for all choices. Our claim follows.

Note that $x_{2}(1)$ and $x_{1}(1) x_{3}(1) x_{4}(1)$ are long, respectively short root elements in the centralizer $C_{G^{\circ}}(\sigma)=G_{2}(k)$ of $\sigma$, with product a regular unipotent element. This thus leads to the example in $G_{2}(k)$ from [7, Ex. 6.1], and we see that in both cases, the generated groups in the generic case agree (see also Proposition 4.12).

Example 3.5. Let $k$ be an algebraically closed field of characteristic 2 and $G=E_{6}(k) .2$, the extension of a simple algebraic group $G^{\circ}=E_{6}(k)$ of type $E_{6}$ by a graph automorphism of order 2. Let $C_{1}$ be the class of long root elements in $G^{\circ}$, with centralizer $U . A_{5}(k)$, where $U$ is unipotent of dimension 21, and $C_{2}$ the class of the graph automorphism $\sigma$ with centralizer $F_{4}(k)$ in $G^{\circ}$. We claim that again $C_{1} C_{2}$ only contains unipotent elements.

Indeed, let $x_{i}(1), 1 \leq i \leq 6$, denote long root elements for the six simple roots, labelled in the standard way (so that node 4 is at the centre of the Dynkin diagram, and $\sigma$ interchanges $\left.x_{3}(1), x_{5}(1)\right)$. So $x:=x_{4}(1) \in C_{1}$, and it's easily seen that $y:=x_{3}(1) x_{5}(1) \sigma$ is conjugate to $\sigma$, so lies in $C_{2}$. The product $x y=x_{4}(1) x_{3}(1) x_{5}(1) \sigma$ lies in a class $D$, with representative $u_{15}$ in [15, p. 160], of dimension 48 (see also [11, Tab. 10]). As $C_{1}$ has dimension 22 and $C_{2}$ has dimension 26, D is dense in $C_{1} C_{2}$ by Lemma 3.1. Again, $\sigma$ normalizes the maximal unipotent subgroup generated by the positive root subgroups, so $\langle x, y\rangle$ is unipotent, and hence by density this holds for all pairs from $C_{1} \times C_{2}$. (Note that both classes have representatives in a Levi subgroup of type $A_{3} .2$, where they correspond to Example 3.2.)

As in the case of $\mathrm{SO}_{8}(k)$, we also recover the example in the connected group $F_{4}(k)$ in characteristic 2 from [7, Ex. 6.3], by considering the centralizer $C_{G^{\circ}}(\sigma)=F_{4}(k)$ of $\sigma$. There, $x_{4}(1)$ is a long root element, and $x_{3}(1) x_{5}(1)$ is a short root element. We see that the group generated by them is isomorphic to the one in $G$. 
We are now ready to prove the main result of this section. Throughout our proofs the following result will make inductive arguments work (see [5, Thm. 1.2]):

Proposition 3.6. Let $G$ be reductive, $H \leq G$ a closed subgroup, and $C, D$ conjugacy classes of $G$.

(a) If $(C \cap H)(D \cap H)$ hits infinitely many semisimple $H$-classes, then $C D$ hits infinitely many semisimple $G$-classes.

(b) If $H$ is reductive and $(C \cap H)(D \cap H)$ hits infinitely many $H$-classes, then $C D$ hits infinitely many $G$-classes.

Theorem 3.7. Let $G$ be an almost simple algebraic group of adjoint type over an algebraically closed field $k$ of characteristic $p \geq 0$ with connected component $G^{\circ}$. Let $C_{1}, C_{2}$ be non-trivial unipotent $G^{\circ}$-classes of $G=\left\langle C_{1}, C_{2}\right\rangle$ such that $x y$ is unipotent for all $x \in C_{1}$, $y \in C_{2}$. Then $p \in\{2,3\}$ and (up to order of $C_{1}, C_{2}$ ) either:

(1) $G=G^{\circ}$ is connected, $C_{1}$ contains long root elements and one of:

(a) $p=2, G=\operatorname{Sp}_{2 n}(k)=\operatorname{Sp}(V)$ with $n \geq 2$, and $C_{2}$ contains involutions $y$ with $(y v, v)=0$ for all $v$ in $V$; or

(b) $(G, p)=\left(F_{4}, 2\right)$ or $\left(G_{2}, 3\right)$, and $C_{2}$ contains short root element; or

(2) $G$ is the extension of $G^{\circ}$ by a graph automorphism of the Dynkin diagram of order $p$, $\mathrm{C}_{2}$ is the class of this quasi-central automorphism, and one of:

(c) $p=2, G=A_{2 n-1}(k) .2$ with $n \geq 2, C_{1}$ consists of long root elements and $C_{2}$ is the class of the graph automorphism with centralizer $C_{n}(k)$;

(d) $p=2, G=D_{n}(k) .2, C_{1}$ contains involutions as given in (1)(a) for $C_{n}(k)$ and $C_{2}$ consists of transvections (the class of graph automorphisms with centralizer $\left.B_{n-1}(k)\right)$;

(e) $p=2, G=E_{6}(k) .2, C_{1}$ is the class of long root elements and $C_{2}$ is the class of the graph automorphism with centralizer $F_{4}(k)$; or

(f) $p=3, G=D_{4}(k) .3, C_{1}$ is the class of long root elements and $C_{2}$ is the class of the graph automorphism with centralizer $G_{2}(k)$.

Moreover, in all these cases, $\langle x, y\rangle$ is unipotent for all $x \in C_{1}, y \in C_{2}$.

Proof. By [7, Thm. 1.1] we may assume that $G$ is disconnected and $C_{2}$, say, lies in $G \backslash G^{\circ}$. So either $p=2$ and $G^{\circ}$ has type $A_{n}(n \geq 2), D_{n}(n \geq 4)$, or $E_{6}$, or $p=3$ and $G^{\circ}$ has type $D_{4}$. By Examples 3.2 3.5 above, all the cases described in (2)(c)-(2)(f) do give examples.

Suppose that $C_{1}$ also consists of outer elements. By Corollary 2.4 there is a unique outer unipotent class lying in the closure of all other unipotent classes in a fixed coset. By taking closures we may assume that $C_{1}, C_{2}$ are these. If $p=3$ and $C_{1}=C_{2}$ then a computation in $\mathrm{SO}_{8}^{+}(3) .3$ shows that the product contains non-unipotent classes. Else, $C_{2}$ contains the inverses of $C_{1}$, and since outer quasi-semisimple elements normalize, but do not centralize, a maximal torus of $G^{\circ}$, we obtain non-trivial semisimple products.

So we may assume that $C_{1} \subset G^{\circ}$. Suppose for the moment that $p=2$.

First consider the case that $G^{\circ}=A_{n}(k), n \geq 2$. Suppose that $C_{1}$ does not consist of transvections. Then by taking closures, we may assume that $C_{1}$ either consists of elements with one Jordan block of size 3 or two Jordan blocks of size 2 , and $C_{2}$ consists of quasi-semisimple elements. Thus, it suffices to work in $A_{2}(k)$ or $A_{3}(k)$. Note that all inner unipotent classes and all classes of involutory graph automorphisms intersect 
$A_{2}(2) .2 \cong \mathrm{PGL}_{2}(7)$ resp. $A_{3}(2) .2 \cong \mathfrak{S}_{8}$ and we obtain a contradiction. So $C_{1}$ consists of transvections. If $n$ is even, we may assume that $C_{2}$ contains quasi-semisimple elements and reduce to the case of $A_{2}(k) .2$. Computing in $A_{2}(2) .2$, we see that there are no examples. If $n \geq 3$ is odd, by [15] all outer unipotent classes except for the quasi-semisimple one, contain the transpose inverse graph automorphism in their closure. So we may reduce to the case of $A_{3}(2) .2$ to see that $C_{2}$ must correspond to transpositions in the symmetric group and so must be the class of the graph automorphisms with centralizer $C_{n}(k)$, the quasi-semisimple class. We thus arrive at case (2)(c).

Next consider the case that $G^{\circ}=D_{n}(k)$. Suppose that $C_{2}$ does not consist of transvections in the natural $2 n$-dimensional representation of $D_{n}(k) .2$. By passing to closures, we may assume that $C_{2}$ contains elements with exactly three nontrivial Jordan blocks of size 2 in the natural $2 n$-dimensional representation. In particular, we see that elements of $C_{2}$ leave invariant a nondegenerate 6-dimensional space. Passing to the closure, we may assume that $C_{1}$ consists of long root elements. In particular, we can reduce to the case of $D_{3}(k)=A_{3}(k)$ for which we already saw that there are no such examples.

So we may assume that $C_{2}$ consists of transvections. We claim that $C_{1}$ contains involutions $x$ so that $(x v, v)=0$ for all $v \in V$, the natural $2 n$-dimensional module for $C_{n}(k) \geq D_{n}(k) .2$ as in $(2)(\mathrm{d})$. If $C_{1}$ consists of involutions which do not have that property, then any $x \in C_{1}$ leaves invariant a nondegenerate 2-dimensional space on which it acts as a transvection. Thus, $x y\left(y \in C_{2}\right)$ can have odd order on that 2-dimensional space.

If $C_{1}$ does not consist of involutions, then going to the closure allows us to assume that $C_{1}$ contains elements of order 4 . It follows that any $x \in C_{1}$ leaves invariant a nondegenerate subspace of dimension 6 or 8 (acting as an element of order 4 ). Thus, we can reduce to either $D_{3}(k)=A_{3}(k)$ or $D_{4}(k)$. In the first case, we are done by the result for type $A$. In the second case, we note that every class of elements of order 4 in $\mathrm{SO}_{8}(k)$ intersects $\mathrm{SO}_{8}^{+}(2)$ and so we may compute structure constants in $\mathrm{GO}_{8}^{+}(2)$ to conclude that there are no such examples.

Now suppose that $G^{\circ}=E_{6}(k)$. First suppose that elements of $C_{2}$ do not have centralizer $F_{4}(k)$. By taking closures, we may assume that $C_{1}$ consists of long root elements in $E_{6}(k)$. By [15, p. 250] all outer unipotent classes except for the quasi-semisimple one contain the graph automorphism with centralizer $C_{4}(k)$ in their closure. Let $\tau$ be an involution with centralizer $F_{4}(k)$. Let $x \in F_{4}(k)$ be a long root element in $E_{6}(k)$ such that $\tau x \in C_{2}$. Then by the result for the connected group $F_{4}(k)$ there is $y \in C_{1} \cap F_{4}(k)$ with $x y$ of odd order.

So we may assume that elements of $C_{2}$ have centralizer $F_{4}(k)$. If $C_{1}$ consists of long root elements then we are in case $(2)(\mathrm{e})$. Else, by taking closures, we may assume that $C_{1}$ is the class $2 A_{1}$ [15, p. 247]. This class has representatives in the Levi subgroup $A_{5}(k)$ and so the result follows by that case.

Finally, consider the case $p=3$ with $G^{\circ}=D_{4}(k)$. Suppose that elements of $C_{2}$ do not have centralizer $G_{2}(k)$. By taking closures, we may assume that $C_{1}$ consists of long root elements. Let $\tau$ be the graph automorphism with centralizer $G_{2}(k)$. Choose $x, y \in G_{2}(k)$ which are long root elements of $D_{4}(k)$ such that $x y$ is not a 3-element (by the result for connected groups) and $\tau x \in C_{2}$. Then $\tau x y$ is not a 3-element either. So we may assume that elements of $C_{2}$ have centralizer $G_{2}(k)$. Note that the graph automorphism fuses the three non-trivial unipotent classes of $G^{\circ}$ of second smallest dimension (see [15, p. 239]), so if $C_{1}$ is not as in case (2)(f), then by closure we may assume that $C_{1}$ is one of these 
classes. But computation of structure constants in the finite subgroup $\mathrm{SO}_{8}^{+}(3) .3$ shows that this does not give an example.

Note that in all the exceptions in the statement, the group generated by $x \in C_{1}, y \in C_{2}$ is always unipotent, by Examples [3.2 [3.5, resp. by the examples in [7]. This completes the proof of Theorem 3.7 .

\section{Products of Conjugacy Classes in Disconnected Algebraic Groups}

In [7, Thm. 1.1] we classified pairs of conjugacy classes $C, D$ in a simple algebraic group such that the product $C D$ consists of a finite union of conjugacy classes (equivalently, the semisimple parts of elements in $C D$ form a single conjugacy class). We build on the results from the previous section to extend this to the disconnected case.

First we give some examples.

Example 4.1. Let $S=\mathrm{SL}_{2 n}(k), n \geq 2$, with $k$ algebraically closed. Let $x \in S$ be any element that is (up to scalar) a pseudoreflection, $y$ a graph automorphism with centralizer $\operatorname{Sp}_{2 n}(k)$ and $G=\langle S, y\rangle$. Then by [7, Ex. 7.2], there is a single $G$-orbit on $x^{S} \times y^{S}$, whence $x^{S} y^{S}$ is a single conjugacy class. In particular, if $k$ is not of characteristic 2 , and $x$ is a 2-element, $\langle x, y\rangle$ is a 2-group.

Example 4.2. Let $G=\mathrm{GO}_{2 n}(k), n \geq 4$, with $k$ algebraically closed. Suppose that $x \in G$ is an element whose centralizer is $\mathrm{GL}_{n}(k)$ and $y \in G$ is a reflection. As $\mathrm{GL}_{n}(k)$ acts transitively on non-degenerate 1-spaces of the natural module for $G$, we have $G=$ $\mathrm{GO}_{2 n-1}(k) \mathrm{GL}_{n}(k)$ and so $G$ has a single orbit on $x^{G} \times y^{G}$. If $k$ is not of characteristic 2 and $x$ has finite order $m$, then $x y^{g}$ will have order $2 m /(2, m)$.

Example 4.3. Let $G=\mathrm{GO}_{2 n}(k), n \geq 4$, with $k$ algebraically closed of characteristic not 2. Suppose that $x$ is unipotent with all Jordan blocks of size at most 2 and $y$ is a reflection in $G$. Then $x$ and $y$ are both trivial on a totally singular subspace of dimension $n-1$ of the natural module. Let $P$ denote the parabolic subgroup stabilizing this space. Then $x$ lies in the unipotent radical of $P$, whence $x y$ has constant semisimple part $y$. Thus we get an example with $C_{1}=x^{G}, C_{2}=y^{G}$.

Example 4.4. Let $G=\mathrm{SO}_{8}(k) .3$ with $k$ algebraically closed of characteristic not 3 . Let $C_{1}$ be the class of root elements of $G^{\circ}$, and $y \in C_{2}$, the class of graph automorphisms with centralizer $H=G_{2}(k)$ in $G^{\circ}$. Let $s \in H$ be an element of order 3 with $C_{H}(s)=\operatorname{SL}_{3}(k)$. By looking at the centralizer in the adjoint action on the Lie algebra of $G^{\circ}$ one sees that $s y$ is $G$-conjugate to $y$. Note that $C_{1} \cap H$ are long root elements in $H$. Thus by [7, Ex. 6.2] the product $\left(C_{1} \cap H\right) s$ contains an element $u s$ with $u$ regular unipotent in $C_{H}(s)=\mathrm{SL}_{3}(k)$, with centralizer dimension 4 in $H$. Thus $x s y \sim u s y$ for some $x \in C_{1}$, with $[u, s y]=1$ and of coprime order. It follows that $\operatorname{dim} C_{G}(x s y)=\operatorname{dim} C_{G}(u s y)=\operatorname{dim} C_{H}(u)=4$, so that $C_{1} C_{2}$ contains a class of $\operatorname{dimension} \operatorname{dim} G-4=24=\operatorname{dim} C_{1}+\operatorname{dim} C_{2}$. An application of Lemma 3.1 shows that $C_{1} C_{2}$ is a union of finitely many classes.

Similarly, let $G=E_{6}(k) .2$ with $k$ algebraically closed of characteristic not 2 . Let $C_{1}$ be the class of root elements of $G^{\circ}$ and $y \in C_{2}$, the class of graph automorphisms with centralizer $H=F_{4}(k)$ in $G$. Let $s \in H$ be an involution with $C_{H}(s)=B_{4}(k)$. By looking at the adjoint action one sees that $s y$ is $G$-conjugate to $y$. Again, $C_{1} \cap H$ consists of long root elements in $H$. Thus by [7, Ex. 6.4] the product $\left(C_{1} \cap H\right) s$ contains elements $u \in B_{4}(k)$ 
whose square is a short root element, with centralizer dimension $30 \mathrm{in} H$. Arguing as before, we see that $C_{1} C_{2}$ contains a class of dimension $\operatorname{dim} G-30=48=\operatorname{dim} C_{1}+\operatorname{dim} C_{2}$, and we conclude by Lemma 3.1 that $C_{1} C_{2}$ is a finite union of classes.

Example 4.5. Let $G=\mathrm{SO}_{8}(k) \cdot \mathfrak{S}_{3}$ with $k$ algebraically closed. Let $C_{1}, C_{2}$ be $G^{\circ}$-classes of graph automorphisms with centralizer $H=B_{3}(k)$ in $G^{\circ}$ lying in two different $G^{\circ}$-cosets. Then $C_{1}, C_{2}$ both have dimension 7 , while any class in the coset of order 3 has dimension at least 14 , which is attained for the quasi-central class of graph automorphisms of order 3 . So the product consists of that single class by Lemma 3.1. See Section 7 for more on this.

Theorem 4.6. Let $G$ be an almost simple algebraic group of adjoint type over an algebraically closed field $k$ of characteristic $p \geq 0$ with connected component $G^{\circ}$. Let $C_{1}$ and $C_{2}$ be nontrivial $G^{\circ}$-conjugacy classes in $G=\left\langle C_{1}, C_{2}\right\rangle$. Suppose that $C_{1} C_{2}$ consists of finitely many conjugacy classes. Then one of the following holds:

(1) $C_{1}, C_{2}$ are unipotent classes and we are in one of the cases of Theorem 3.7; or

(2) $G=G^{\circ}$ is connected, $C_{2}$ is unipotent, and one of

(a) $G=\operatorname{Sp}_{2 n}(k), n \geq 2, p \neq 2, C_{1}$ contains involutions and $C_{2}$ contains long root elements;

(b) $G=\mathrm{SO}_{2 n+1}(k), n \geq 2, p \neq 2, C_{1}$ contains reflections (modulo scalars) and $C_{2}$ contains unipotent elements with all Jordan blocks of size at most 2;

(c) $G=F_{4}, p \neq 2, C_{1}$ contains involutions with centralizer $B_{4}(k)$ and $C_{2}$ contains long root elements;

(d) $G=G_{2}, p \neq 3, C_{1}$ contains elements of order 3 with centralizer $\mathrm{SL}_{3}(k)$ and $C_{2}$ contains long root elements; or

(3) $G$ is disconnected, $C_{2}$ contains quasi-semisimple graph automorphisms, and one of

(e) $\left[G: G^{\circ}\right]=2, G^{\circ}=A_{2 m-1}(k), m>1, C_{1}$ contains (semisimple) pseudoreflections and $C_{2}$ contains graph automorphisms with centralizer $C_{m}(k)$;

(f) $\left[G: G^{\circ}\right]=2, G^{\circ}=A_{2 m-1}(k)$ with $p \neq 2, m>1, C_{1}$ contains transvections and $C_{2}$ contains graph automorphisms with centralizer $C_{m}(k)$;

(g) $\left[G: G^{\circ}\right]=2, G^{\circ}=D_{n}(k), n \geq 4, C_{1}$ contains semisimple elements with centralizer $A_{n-1}(k)$ and $C_{2}$ contains graph automorphisms with centralizer $B_{n-1}(k)$;

(h) $\left[G: G^{\circ}\right]=2, G^{\circ}=D_{n}(k)$ with $p \neq 2, n \geq 4, C_{1}$ contains unipotent elements with all Jordan blocks of size at most 2 and $C_{2}$ contains graph automorphisms with centralizer $B_{n-1}(k)$;

(i) $\left[G: G^{\circ}\right]=2, G^{\circ}=E_{6}(k)$ with $p \neq 2, C_{1}$ contains long root elements and $C_{2}$ contains graph automorphisms with centralizer $F_{4}(k)$;

(j) $\left[G: G^{\circ}\right]=3, G^{\circ}=D_{4}(k)$ with $p \neq 3, C_{1}$ contains long root elements and $C_{2}$ contains graph automorphisms with centralizer $G_{2}(k)$; or

(k) $\left[G: G^{\circ}\right]=6, G^{\circ}=D_{4}(k), C_{1}$ and $C_{2}$ are classes in different cosets modulo $G^{\circ}$ of graph automorphisms of order 2.

Note that cases (f), (h), (i) and (j) are direct analogues of the unipotent pairs in Theorem 3.7. We analyze the case 3(k) completely in Section 7 , see Proposition 17.4, If we consider $G$-classes instead of $G^{\circ}$-classes, we get the same examples except for the ones in $3(\mathrm{k})$.

The proof of Theorem 4.6 will be given in a series of five lemmas. First note that if $G$ is connected, the result was shown in [7, Thm. 1.1]. So we may and will assume that $G^{\circ}$ 
is one of $A_{n}(k)(n \geq 2), D_{n}(k)(n \geq 4)$, or $E_{6}(k)$. Let $r=\left[G: G^{\circ}\right]$. We may assume that $C_{2}$ consists of outer automorphisms. We consider various cases.

Lemma 4.7. The claim in Theorem 4.6 holds when neither of the classes $C_{1}, C_{2}$ is contained in $G^{\circ}$.

Proof. Passing to closures, we may first assume by Theorem 2.3 that $C_{1}, C_{2}$ are both quasisemisimple. Then there exist $(x, y) \in C_{1} \times C_{2}$ normalizing a common Borel subgroup $B$ and a maximal torus $T \leq B$. Let $S:=[y, T]$. Then $y S \subseteq C_{2}$ and $S$ is infinite as $y \notin C_{G}(T)=C_{G^{\circ}}(T)$. Let $m$ denote the order of $z=x y$ in $G / G^{\circ}$. If $m=1$ then $x y S \subset T \cap C_{1} C_{2}$ is infinite, so contains infinitely many classes.

If $m>1$ then necessarily $G^{\circ}=D_{4}(k)$. If $m=2$ then $(x y t)^{2}=z^{2} t^{z} t$, for $t \in S$, is finite only if $z$ acts as -1 on $S$. Note that this configuration can only occur when $C_{1}, C_{2}$ lie in cosets of $G^{\circ}$ of order 2, 3 respectively. Explicit computation shows that here $z$ does not act as -1 on $S$.

Thus we have that $m=3$. If $C_{1}, C_{2}$ lie in the same coset of order 3 , then $(x y t)^{3}=$ $\left\{z^{3} t^{z^{2}} t^{z} t \mid t \in S\right\}$. Again, explicit computation shows that this is not finite for suitable $N_{G}(T)$-conjugates of $x, y$. The only remaining possibility is that $G / G^{\circ} \cong \mathfrak{S}_{3}, x, y$ lie in two different cosets of order 2 , as in $(3)(\mathrm{k})$ of the conclusion.

Lemma 4.8. Let $G=A_{1}(k) \backslash Z_{r}$, the wreath product of $A_{1}(k)$ with the cyclic group of prime order $r$. Let $C_{1} \subset G^{\circ}$ be a conjugacy class whose projection to at least two factors is non-central, and $C_{2} \subset G \backslash G^{\circ}$ any conjugacy class. Then:

(a) $C_{1} C_{2}$ consists of infinitely many conjugacy classes.

(b) $\left[C_{1}, C_{2}\right]$ consists of infinitely many conjugacy classes.

Proof. Let $\sigma$ denote a generator of the cyclic subgroup of $G$ permuting the $A_{1}(k)$-factors. Any outer element is conjugate to one of the form $\sigma(1, \ldots, 1, x)$ for some $x \in A_{1}(k)$. An easy computation with such elements shows (a) and (b), using that the product of any two non-central classes of $A_{1}(k)$ meets infinitely many classes.

Lemma 4.9. The claim in Theorem 4.6 holds when $G^{\circ}=A_{n}(k)$.

Proof. By our initial reductions and Lemma 4.7 we may assume that $r=2, C_{1} \subset G^{\circ}$ and $C_{2}$ is outer.

Case 1. $n$ is even.

In this case, we claim there are no examples. By taking closures, we may assume that $C_{2}$ contains quasi-semisimple elements. Thus $x_{2} \in C_{2}$ normalizes a maximal torus, hence any Levi subgroup $L$ of $G^{\circ}$ invariant under the graph automorphism. Again by closure, $C_{1}$ consists of semisimple elements or of transvections. In either case, we see that one can find $x_{i} \in C_{i}$ normalizing a subgroup $A_{2}(k)$ and acting nontrivially. Thus, it suffices to take $n=2$. Now any quasi-semisimple element of $A_{2}(k)$ normalizes a maximal torus, so is of the form $x t, t \in T$, for some fixed quasi-semisimple element $x$. It is then a straightforward matrix computation to see that $C_{1} C_{2}$ always meets elements with distinct characteristic polynomial, so with distinct semisimple part.

Case 2. $n=2 m-1 \geq 3$ is odd.

Arguing as above we can reduce to $x_{1}, x_{2}$ lying in a Levi subgroup of type $A_{3}$. Inspection shows that then $x_{2}$ has to be an involution. We claim that $C_{2}$ consists of involutions with 
centralizer $C_{m}(k)$. Suppose not. Then by [3], Tab. 4.5.1], elements in $C_{2}$ have centralizer $D_{m}(k)$. We may assume that $x_{1} \in C_{1}$ is either semisimple or a transvection, and then after conjugation we have $x_{1}, x_{2}$ in a disconnected subgroup with connected component a Levi subgroup of type $A_{n-1}$, where no example exists by the first case.

If $x_{1}$ is semisimple, there is some root of $G$ which is non-trivial on $x_{1}$. Since $G^{\circ}$ has just one root length, after conjugation we may assume that $x_{1}$ lies in an $x_{2}$-stable Levi subgroup $L$ of type $D_{2}$ but not in its center, with $x_{2}$ swapping the two factors. Moreover, if no eigenspace of $x_{1} \in C_{1}$ has dimension $n-1$, then we may arrange that the image of $x_{1}$ in both factors is non-trivial. In that case, by Lemma 4.8 the product $C_{1} C_{2}$ meets infinitely many semisimple classes inside the wreath product $A_{1}(k)<2$. On the other hand, if $x_{1}$ is a pseudo-reflection (modulo scalars), we get case (3)(e) of the assertion by Example 4.1.

If $x_{1}$ is unipotent, we may assume that $p>2$ since otherwise we are in the situation of Theorem 3.7. If $x_{1}$ is a transvection, then we get case (f) by Example 3.2. Else, by closure we may assume that $x_{1}$ has two Jordan blocks of size 2 or one of size 3 . In the first case, we may reduce to $A_{3}$, in the second to $A_{2}$, and no examples arise.

If $x_{1}$ is neither unipotent nor semisimple, then by the previous arguments it must be the commuting product of a pseudo-reflection with a transvection. In this case, we may again reduce to the wreath product $A_{1}(k)<2$ and apply Lemma 4.8 .

Lemma 4.10. The claim in Theorem 4.6 holds when $G^{\circ}=D_{n}(k), n \geq 4$.

Proof. As in Lemma 4.9 we may assume that $C_{1} \subset G^{\circ}$ and $C_{2}$ is outer, so $r \in\{2,3\}$.

Case 1. $r=2$.

First suppose that $x_{1}$ is semisimple but not as in the conclusion (g). By taking closures we may assume $x_{2}$ is quasi-semisimple. Then we can reduce to $\mathrm{GO}_{4}(k)=A_{1}(k) \prec 2$. Moreover $x_{1}$ projects to elements which are nontrivial in each factor $A_{1}(k)$ whence $C_{1} C_{2}$ contains infinitely many classes by Lemma 4.8. Next suppose that $x_{1}$ has centralizer $\mathrm{GL}_{n}(k)$. If $x_{2}$ is quasi-semisimple but not a reflection, we can reduce to $D_{3}(k)=A_{3}(k)$ and invoke Lemma 4.9. So we may assume that the semisimple part of $x_{2}$ is a reflection. If its unipotent part is nontrivial, then by taking closures we may assume it is a root element in $\mathrm{SO}_{2 n-1}(k)$ and so either has two Jordan blocks of size 2 or one Jordan block of size 3. Again, we can reduce to $D_{3}(k)$ where this cannot happen. When $x_{2}$ is a reflection, we arrive at $(3)(\mathrm{g})$ by Example 4.2 ,

If $x_{1}$ is unipotent with all Jordan blocks of size at most 2 and $x_{2}$ is a reflection, then this gives $(3)(\mathrm{h})$ by Example 4.3. If $x_{2}$ does not consist of reflections, we may again reduce to $D_{3}(k)$ to obtain a contradiction. If $x_{1}$ has a Jordan block of size at least 3 , by taking closures we may assume that it has just one Jordan block and $x_{2}$ is semisimple, and then we may reduce to a subgroup $D_{3}(k)$ for which there are no such examples.

Finally, suppose that $x_{1}$ is a mixed element. The above argument shows that $x_{2}$ is a reflection, the semisimple part of $x_{1}$ has centralizer $\mathrm{GL}_{n}(k)$ and its unipotent part has Jordan blocks of size at most 2. Again, we can reduce to $D_{3}(k)$ to rule out this case.

Case 2. $r=3$, so $n=4$.

First assume that $x_{2} \in C_{2}$ is quasi-semisimple. If $x_{1}$ is unipotent, then $x_{1}, x_{2}$ lie in the normalizer of a maximal parabolic subgroup $P$ of type $A_{1}^{3}$. If $x_{1}$ is not a long root element, 
the image of $x_{1}$ in the Levi subgroup $L$ of $P$ is non-trivial in at least two of the factors, whence the product $C_{1} C_{2}$ meets infinitely many classes in $L$ by Lemma 4.8. If $x_{1}$ is a long root element, we may conjugate $x_{1}, x_{2}$ into the graph centralizer $G_{2}(k)$. By [7, Thm. 5.11] the only example there is for $x_{2}$ to be the 3-element with centralizer $\mathrm{SL}_{3}(k)$. But by the argument in Example 4.4, all such elements fuse into the class of graph automorphisms with centralizer $G_{2}(k)$, so we arrive at $(3)(\mathrm{j})$.

Any non-central semisimple element has a conjugate which is non-trivial in at least two factors of the Levi subgroup $L$ of type $A_{1}^{3}$ and thus gives infinitely many classes there. Finally, if $x_{1}$ is a mixed element, we may assume that its unipotent part is a long root element, and then again its image in $L$ is non-trivial in at least two factors.

Finally, if $x_{2}$ is not quasi-semisimple, by the previous argument and taking closures, we may assume that $x_{2}=x u=u x$ where $u$ is a long root element and $x$ is quasi-semisimple of order 3 , and $x_{1}$ is a long root element. In particular if we take $x_{1}$ centralizing $x$, we see that $x_{1}$ and $u$ are conjugate in $G_{2}(k)$ and since $u^{G_{2}(k)} u^{G_{2}(k)}$ hits infinitely many classes, we are done.

Lemma 4.11. The claim in Theorem 4.6 holds when $G^{\circ}=E_{6}(k)$.

Proof. Here we have $r=2$. By closure we may assume that $x_{1} \in C_{1}$ is semisimple or unipotent of type $A_{2}$, or with unipotent part a long root element, and $x_{2} \in C_{2}$ is quasisemisimple. Hence we may arrange so that $x_{1}, x_{2}$ are contained in a Levi subgroup of type $A_{5}$, which is normalized by the graph automorphism. By Lemma 4.9 this forces $x_{2}$ to be an involution. There are two classes of outer involutions in $G$, with centralizers $F_{4}(k)$ resp. $C_{4}(k)$. The outer involutions of $A_{5}(k) .2$ with centralizer of type $D_{3}$ fuses into the outer class with centralizer of type $C_{4}$ (since $A_{5}(k)$ has composition factors of dimensions $6,6,15$ on the 27-dimensional module for $E_{6}(k)$, and so has $D_{3}(k)$, while $F_{4}(k)$ has a 1-dimensional constituent), so by our considerations in Lemma 4.9, $x_{2}$ must have centralizer $F_{4}(k)$, and $x_{1}$ must be a long root element. This occurs by Example 4.4.

We have now discussed all possibilities for $G$ and thus completed the proof of Theorem 4.6 .

We note the following relation between the examples in disconnected groups in Theorem 4.6 and the ones in connected groups in [7, Thm. 1.1]:

Proposition 4.12. Let $G$ be a simple algebraic group, $\tau$ a graph automorphism of $G$ with reductive centralizer $H=C_{G}(\tau)$. Suppose moreover that for some $1 \neq g \in H, \tau$ and $g \tau$ are $G$-conjugate. If $C:=\tau^{G}$ and $D \subset G$ are classes such that $C D$ consists of only finitely many classes in $G \tau$, then the same holds for $C_{1}=g^{H}, D_{1}=D \cap H$ in $H$.

Proof. Clearly, $C_{1} D_{1} \tau=g^{H} \tau D_{1}=(g \tau)^{H} D_{1} \subset C D$, and if the latter meets only finitely many $G$-classes, then the former only meets finitely many $H$-classes, see Proposition 3.6.

This applies to cases (e), (f), (h), (i) and (j) in Theorem 4.6.

\section{Commutators}

In this section we turn to commutators of pairs of conjugacy classes. We first investigate the commutator of a single class and thus prove Theorem 1.4. The proof follows the ideas in $[7, \S 5]$. 
It is convenient to recall the following result [4] (which answered a question of Katz before it was asked - see [8, 2.16.7]).

Theorem 5.1. Let $G$ be a simple algebraic group of type $A$ or $C$. Suppose that $x, y \in G$ with $[x, y]$ a long root element (i.e., a transvection). Then $\langle x, y\rangle$ is contained in a Borel subgroup.

Proof. We may as well work in $\mathrm{SL}_{n}$. Suppose that $z:=x^{-1} y^{-1} x y$ is a long root element. Write $z=I+N$ where $N$ is a nilpotent rank one matrix. Then $x y-y x=y x N$ is a rank one matrix. Now apply the main result of [4].

Proof of Theorem 1.4. Let $G$ be an almost simple algebraic group over $k$ and $C$ a $G^{\circ}$ conjugacy class of $G$ outside $Z\left(G^{\circ}\right)$. Let $w: C \times C \rightarrow G$ be the map defined by $w(x, y)=$ $[x, y]$.

Since $C$ is an irreducible variety, if the result fails, the semisimple part of $[x, y]$ for $(x, y) \in C \times C$ would be constant. Of course, $1=[x, x]$ and therefore the image of $w$ must be contained in the set of unipotent elements of $G$. If $[C, C]$ consists of finitely many classes, the same is true for its closure, so we may also replace $C$ by any class in its closure and so assume that $C$ is semisimple or unipotent.

First suppose that $G \cong A_{1}(k)$. We claim that it suffices to show the result for $\mathrm{SL}_{2}(k)$. Indeed, lift $C$ to a class of $\mathrm{SL}_{2}(k)$; the image of $w$ is irreducible and contains 1 , so still all commutators are unipotent (not just unipotent modulo the center).

Choose $(x, y) \in C \times C$ so that $x$ and $y$ are not contained in a common Borel subgroup (since any non-central element lies in at most two Borel subgroups, this clearly can be done). By Theorem [5.1, $[x, y]$ is not unipotent.

Next suppose that $G=G^{\circ}$ is connected. If $C$ contains semisimple elements, we can choose $x \in C \cap T$ where $T$ is a maximal torus and so $x$ does not centralize some root subgroup $U$. Let $U^{-}$be the corresponding negative root subgroup. Then $\left\langle T, U, U^{-}\right\rangle$is a product of a torus with an $A_{1}(k)$, whence the result follows.

If $C$ is unipotent, by closure we may assume that it consists of root elements. Then we just work in $\left\langle U, U^{-}\right\rangle$where $U$ is a root subgroup generated by elements of $C$ and again the result follows by the $A_{1}(k)$ result.

Finally suppose that $G$ is disconnected. We may assume that $C$ consists of outer automorphisms, and by closure that it is closed. In particular, elements of $C$ are quasisemisimple by Theorem 2.3. By definition, any such element normalizes, but does not centralize, a maximal torus of $G^{\circ}$, and thus there exist non-trivial semisimple commutators of elements in $C$.

It follows easily from the previous result that if $C$ is a noncentral conjugacy class of $\ell$-elements for some prime $\ell$, then commutators of pairs of elements of $C$ cannot consist only of $\ell$-elements (this is related to the question of Shumyatsky).

If we take distinct classes, there are examples in [7] showing that the commutators could always be unipotent. Arguing as in [7], we can actually classify all such pairs.

Theorem 5.2. Let $G$ be an almost simple algebraic group over an algebraically closed field $k$ of characteristic $p \geq 0$. Let $C, D$ be non-central $G^{\circ}$-conjugacy classes in $G$ with $G=\langle C, D\rangle$. Assume that $C, D$ do not lie in distinct cosets of order 2 if $G=D_{4}(k) \cdot \mathfrak{S}_{3}$. 
Then

$$
[C, D]:=\{[x, y] \mid x \in C, y \in D\}
$$

is the union of finitely many conjugacy classes if and only if $C, D$ are as given in Theorem 3.7 or as in Theorem 4.6(a)-(j). In particular, $C \neq D$ in this case.

Proof. Let $w: C \times D \rightarrow G$ be as in the previous proof. By irreducibility the semisimple part of elements in $[C, D]$ has to be constant.

We can now essentially follow the proof in [7], respectively the proofs of Theorems 3.7 and 4.6. First assume that $G$ is connected. For $G=\mathrm{SL}_{2}(k)$, choosing $(x, y) \in C \times D$ in the same Borel subgroup, $[x, y]$ is clearly unipotent. On the other hand, choose $x$ and $y$ so that they are not contained in a common Borel subgroup. Then by Theorem [5.1, $[x, y]$ is not unipotent. So there's no example for $\mathrm{SL}_{2}(k)$.

If $[C, D]$ consists of finitely many classes, the same is true for its closure, so we may also replace $C$ and $D$ by any classes in their closures and so assume that each is semisimple or unipotent. Moreover, if one of them is a unipotent class, we may assume that it consists of root elements. We can now argue exactly as in the proof of [7, Thm. 5.11] to rule out every configuration apart from those in [7, Thm. 5.11(2)-(6)], except for the case that $p \neq 2, G=\operatorname{Sp}_{4}(k)$, and the semisimple part of $c$ is an involution. But again by working inside the subsystem subgroup $A_{1}(k)^{2}$ we see that $[C, D]$ can have infinitely many distinct semisimple parts by Lemma 4.8.

In all the exceptions of [7, Thm. 5.11(2)-(6)], any pair $(x, y) \in C \times D$ lies in some common Borel subgroup of $G$ by [7, Cor. 5.14], whence the commutator $[x, y]$ is unipotent and so $[C, D]$ consists of finitely many (unipotent) classes.

Now assume that $G$ is not connected, but $C, D$ are unipotent. Then the same would have to hold for all classes in $[C, D]$, since all unipotent elements have conjugates in a maximal unipotent subgroup of the normalizer of a Borel subgroup. Following the arguments in the proof of Theorem 3.7] we see that the assertion follows once we show that the only pairs of 2 -power classes in $\mathfrak{S}_{8}, \mathrm{PGL}_{2}(7), \mathrm{GO}_{8}^{+}(2)$ with commutator consisting of 2-elements are those in the conclusion. This can be checked by direct computation.

Finally assume that $G$ is disconnected and not both $C, D$ are unipotent. Here, we go through the proof of Theorem 4.6. If both classes are outer (and quasi-semisimple), then we may find representatives $x, y$ normalizing a maximal torus $T$, as in the proof of Lemma 4.7. First assume that $x, y$ lie in the same cyclic subgroup of $G / G^{\circ}$. Then with $S=\left[T, y^{-1}\right]$ and $R=[x, S]$ we have $[x, S y]=\left\{x^{-1} y^{-1} x r y \mid r \in R\right\} \subseteq\left[C_{1}, C_{2}\right] \cap T$, which is always infinite, since $x$ may be chosen not to centralize the commutator space of $y^{-1}$. The only remaining case is when $G=D_{4} \cdot \mathfrak{S}_{3}$ with $x, y$ in non-trivial cosets of different order. Here, the cube of the commutator $[x, S y]$ hits infinitely many classes in $T$ since $\left\{r \in R \mid r^{z^{2}} r^{z} r\right\}$ is not finite, for $z=\left[y^{-1}, x\right]$.

Thus, $C$ is inner, say. As in the proof of Lemma 4.9 we are done for $A_{n}(k)$ once we have shown the claim for $n=2$ and $n=3$, where it is a direct matrix calculation. The arguments for all other types go through unchanged.

In the case of commutators between $G^{\circ}$-classes in two different cosets of order 2 in $D_{4}(k) \cdot \mathfrak{S}_{3}$, there are many further examples, see Example 7.6 below. 


\section{Double cosets, Products and Commutators}

The aim of this section is the Characterization Theorem 1.1 for various finiteness properties. For this we need to investigate some of the examples from the previous three sections a bit more closely.

Proposition 6.1. Let $G=\mathrm{GO}_{2 n}(k), n \geq 3$, with $k$ algebraically closed of characteristic $p$. Let $x \in G^{\circ}$ be unipotent such that either

(1) $p \neq 2$ and all Jordan blocks of $x$ have size at most 2 ; or

(2) $p=2, x^{2}=1$ and $(x v, v)=0$ for all $v$ in the natural module $V$ for $G$ (under the associated alternating form).

Then $C_{G}(x)$ has at most two orbits on nondegenerate 1-spaces in $V$.

Proof. We claim that any nondegenerate 1-space is conjugate under $C_{G}(x)$ to an element in a nondegenerate 4 -space or 6 -space that is $x$-invariant.

Write $V=V_{1} \perp V_{2}$ where $V_{1}$ and $V_{2}$ are $x$-invariant and $x$ is trivial on $V_{1}$ and has all Jordan blocks of size exactly 2 on $V_{2}$.

It suffices to deal with each space separately. In the first case the centralizer contains $\mathrm{GO}\left(V_{1}\right)$ and we can move any 1 -space into any nondegenerate 2 -space. So we need to consider $V_{2}$. We claim that any vector is $C_{G}(x)$-conjugate to an element of some $x$ invariant nondegenerate 4 -space.

In both cases the fixed space of $x$ contains a maximal totally singular isotropic space. Thus we can write $V_{2}=U \oplus U^{\prime}$ where $U$ and $U^{\prime}$ are maximal complementary totally isotropic spaces, with $U$ the fixed space of $x$. Thus $x$ is trivial on $V_{2} / U$ as well and so $x$ is in the unipotent radical $Q$ of the stabilizer of $U$. Then $x$ corresponds to a full rank skew symmetric matrix in $Q$. Thus, the centralizer of $x$ is $Q \cdot \operatorname{Sp}(U)$ with $\operatorname{Sp}(U)$ acting isomorphically on both $U$ and $U^{\prime}$. Since $\operatorname{Sp}(U)$ is transitive on non-zero vectors of $U$, it is easy to see that given $v=u+u^{\prime}$, we can move $u, u^{\prime}$ into a nondegenerate $x$-invariant 4-space.

Thus we are reduced to $\mathrm{GO}_{4}(k)$ and $\mathrm{GO}_{6}(k)$. It is straightforward to check that in the first case there is a unique orbit, while in the second case there are two orbits (as can be seen for example by checking that over finite fields $\mathbb{F}_{q}$, there are two orbits, of lengths $\left.q^{3}\left(q^{2}-1\right), q^{2}(q-1)\right)$.

\section{Proposition 6.2.}

(a) Let $G=D_{4}(k) .3$, the extension by a graph automorphism, and $x \in G^{\circ}$ a long root element. Then $\left|G_{2}(k) \backslash G^{\circ} / C_{G^{\circ}}(x)\right|=5$ and $\left|G_{2}(k) \backslash G / C_{G}(x)\right|=3$.

(b) Let $G=E_{6}(k) .2$, the extension by a graph automorphism, and $x \in G^{\circ}$ a long root element. Then $\left|F_{4}(k) \backslash G / C_{G}(x)\right|=2$.

Proof. First consider $G=D_{4}(k) .3$, the extension by the graph automorphism $\sigma$ of order 3 . Let $B$ be a $\sigma$-stable Borel subgroup of $G^{\circ}$. Let $P$ be the normalizer in $G^{\circ}$ of a $\sigma$-stable root subgroup, a maximal parabolic subgroup of type $A_{1}^{3}$. Its derived subgroup $P^{\prime}$ is the centralizer of a long root element in $G^{\circ}$. In order to show that $G_{2}(k) \backslash G / P^{\prime}$ is finite, it suffices to see that $G_{2}(k)$ has finitely many orbits on the set of long root elements of $G^{\circ}$. We claim that there are precisely five orbits. Clearly, there's one orbit of long root elements (in $B$ ) centralized by $\sigma$; these are long root elements in $H=C_{G^{\circ}}(\sigma) \cong G_{2}(k)$ 
and thus have centralizer $Q^{\prime}$ in $H$, where $Q$ is a maximal parabolic subgroup of $H$. Furthermore, there are three orbits of long roots fused under $\sigma$, such that the product over any $\sigma$-orbit is a short root element in $H$. These have centralizer $U_{3} \cdot A_{1}(k)$, with $U_{3}$ unipotent of dimension 3. Finally, let $x \in U=R_{u}(B)$ be the product of a root element with support 2 (on the simple roots of $G^{\circ}$ with respect to $B$ ) with a commuting root element with support 3 . Then the centralizer of $u$ in $U \cap H$ has dimension 4 . The product $u u^{\sigma} u^{\sigma^{2}}$ is a unipotent element $\left(x_{a+b}(1) x_{2 a+b}(1)\right.$ with respect to the simple roots $a, b$ of $H$ ) with 4-dimensional unipotent centralizer in $H$. Thus $\operatorname{dim}\left(C_{H}(u)\right)=4$. (This is the element constructed in Example 4.4 when $p \neq 3$, respectively in Example 3.4 when $p=3$.)

For $p>0$, over the field with $q=p^{a}$ elements the corresponding $G_{2}(q)$-orbits in $D_{4}(q) .3$ have lengths $q^{6}-1, q^{2}\left(q^{6}-1\right)(3$ times $)$, and $q^{2}\left(q^{2}-1\right)\left(q^{6}-1\right)$, which adds up to the number of long root elements, so we've accounted for all orbits in this case. By arguing as in [7, Ex. 6.2] we conclude that the claim holds over any algebraically closed field. Note that since $\sigma$ permutes the three orbits of dimension 8, we obtain three orbits under $C_{G}(\sigma)$.

Now consider $G=E_{6}(k) .2$, the extension by the graph automorphism $\sigma$ of order 2 . The centralizer of a long root element in $G^{\circ}$ is the derived subgroup $P^{\prime}$ of a parabolic subgroup $P$ of $G^{\circ}$ of type $A_{5}$. The group $H=C_{G^{\circ}}(\sigma) \cong F_{4}(k)$ has one orbit on long root elements contained in $H$, so centralized by $\sigma$, with centralizer $Q^{\prime}$, where $Q$ is an end-node parabolic subgroup of $H$. Now let $x$ be a long root element not fixed by $\sigma$, corresponding to the root $\alpha=\sum_{i=1}^{6} a_{i} \alpha_{i}$, with $\alpha_{1}, \ldots, \alpha_{6}$ the simple roots of $E_{6}$ in the standard numbering and $\left(a_{1}, \ldots, a_{6}\right)=(1,1,1,2,2,1)$. This root subgroup is normalized by a maximal torus of $H$, and also centralized by all but three positive root subgroups in $H$. Moreover, $\alpha$ is centralized by a subgroup of type $W\left(A_{3}\right)$ in the Weyl group of $H$. Thus $C_{H}^{\circ}(x)=U \cdot A_{3}(k)$ with $U$ unipotent of dimension 15 , and the $H$-orbit of $x$ has dimension $22=\operatorname{dim} E_{6}(k)-\operatorname{dim} P^{\prime}$, so it is the dense orbit. Note that the $\sigma$-conjugate root element $x^{\sigma}$ (in the root subgroup corresponding to (112211)) has exactly the same connected centralizer in $H$, and for $p \neq 2, C_{H}\left(x x^{\sigma}\right)=U \cdot A_{3}(k) .2$ is an extension of $C_{H}^{\circ}(x)$ of degree 2 (see [14]). Since $W\left(F_{4}\right)$ contains an element interchanging the root subgroups of $x$ and $x^{\sigma}$, we deduce that $C_{H}(x)=U \cdot A_{3}(k)$ is connected for $p \neq 2$. A direct computation in $E_{6}(2)$ shows that $C_{H}(x)$ is also connected in characteristic 2.

In the finite group over $\mathbb{F}_{q}$, the lengths $\left(q^{4}+1\right)\left(q^{12}-1\right)$ and $q^{3}\left(q^{4}+1\right)\left(q^{12}-1\right)$ of these two orbits add up to the total number of long root elements. We conclude as in the previous case.

We now turn to the proof of Theorem 1.1 from the introduction. One step is given by the following result:

Lemma 6.3. Let $G$ be an almost simple algebraic group over an algebraically closed field, and let $C, D$ be $G^{\circ}$-classes. If every $\left(x_{1}, x_{2}\right) \in C \times D$ normalizes some Borel subgroup of $G^{\circ}$ then $C D$ is a finite union of $G^{\circ}$-conjugacy classes.

Proof. Let $\left(x_{1}, x_{2}\right) \in C \times D$ normalize the Borel subgroup $B$ of $G^{\circ}$. If $x_{1}$, say, is inner, then conjugates of $x_{1}$ inside $B$ have only finitely many distinct semisimple parts, so the possible products with $x_{2}$ have only finitely many quasi-semisimple classes in their 
closures, whence we get (i) by [7, Lemma 5.1] (note the argument shows that the number of possible quasi-semisimple classes is independent of the Borel subgroup).

So now let's assume that $C$ and $D$ are both outer. We claim that this cannot occur aside from the case that $C$ and $D$ are in distinct cosets of outer involutions (whence $\left.G=D_{4}(k) \cdot \mathfrak{S}_{3}\right)$.

Note that under the assumption that every pair in $C \times D$ normalizes a Borel subgroup, the conclusion holds if and only if it holds for $C^{\prime} \times D^{\prime}$ where $C^{\prime}$ and $D^{\prime}$ are the closed quasi-semisimple classes in $\bar{C}$ and $\bar{D}$ respectively. So it suffices to assume that $C$ and $D$ are both quasi-semisimple. Note that our condition is also true for powers of $C$ and $D$.

By choosing a parabolic subgroup that is invariant under the graph automorphism and such that it still induces a graph automorphism on its Levi subgroup, we can reduce to the cases $A_{1}(k)^{2}, A_{2}(k)$ or $A_{1}(k)^{3}$ (the latter in $D_{4}(k)$ ). It is straightforward in each of these cases to check that there is not always a common Borel subgroup unless possibly $C$ and $D$ are in distinct cosets of graph automorphisms of order 2. Moreover, in the latter case, if neither $C$ nor $D$ have order 2, then one sees (as in the proof of Proposition 7.4), there will not always be a common invariant Borel subgroup. So we may assume that $C$ consists of graph automorphisms of order 2. Similarly, if $D$ is not as given in Proposition 7.4(1), there will not always be a common invariant Borel subgroup. If $D$ does satisfy Proposition 7.4(1), then in fact there are only finitely many classes in $C D$.

We are now ready to prove Theorem 1.1, which is part of the following statement:

Theorem 6.4. Let $G$ be an almost simple algebraic group over an algebraically closed field, and let $C, D$ be $G^{\circ}$-classes in $G$. The following are equivalent:

(i) $C D$ is a finite union of $G^{\circ}$-conjugacy classes.

(ii) The closure of $C D$ contains a unique quasi-semisimple conjugacy class.

(iii) $C_{G^{\circ}}\left(x_{1}\right) \backslash G^{\circ} / C_{G^{\circ}}\left(x_{2}\right)$ is finite for all $\left(x_{1}, x_{2}\right) \in C \times D$.

(iv) $G^{\circ}$ has finitely many orbits on $C \times D$.

(v) $\left\langle x_{1}, x_{2}\right\rangle$ normalizes some Borel subgroup of $G^{\circ}$ for every $\left(x_{1}, x_{2}\right) \in C \times D$.

(vi) $C_{G^{\circ}}\left(x_{1}\right) C_{G^{\circ}}\left(x_{2}\right)$ is dense in $G^{\circ}$ for some $\left(x_{1}, x_{2}\right) \in C \times D$.

Moreover, these conditions imply, and if $G \neq D_{4} \cdot \mathfrak{S}_{3}$ are all equivalent to

(vii) $[C, D]$ is a finite union of $G^{\circ}$ conjugacy classes.

Proof. Statements (i) and (ii) are equivalent by Corollary 2.4 and clearly (iii) and (iv) are equivalent. Furthermore, (iii) implies (vi). By [7, Lemma 5.3] we have that (vi) implies (ii).

It is shown in Lemma 6.3 that (v) implies (i). The equivalence of (i) and (vii) is the statement of Theorem 5.2 .

Thus it remains to show that (i) implies (iii) and (v), which we do by going through the cases in Theorem 4.6. For the cases in (1), this is [7, Cor. 5.14]. The unipotent examples in (2) are those from Theorem 3.7 (2)-(5). Here, it is shown in Examples 3.2, 3.4, 3.5, and in [7, Ex. 6.6] that $\left\langle x_{1}, x_{2}^{g}\right\rangle$ is a unipotent subgroup and thus (v) holds. Furthermore, we have (iii) by Example 3.2 and Propositions 6.1 and 6.2.

Now let's turn to the non-unipotent examples in Theorem 4.6(e)-(k). For case (e), there is just one orbit of $G$ on $x_{1}^{G} \times x_{2}^{G}$ by [7, Ex. 7.2], and clearly we can find a pair in the normalizer of some Borel subgroup, whence (iii) and (v) hold. In case (f), we have just 
two orbits by Example 3.2. Since clearly the graph automorphism $\sigma$ cannot centralize all transvections in a $\sigma$-stable Borel subgroup of $G^{\circ}$, we get representatives of both orbits in the normalizer.

In case (g), by Example 4.2 there's again just a single orbit. In case (h), by Example 4.3 both elements always lie in the normalizer of some parabolic subgroup, and $x_{1}$ in its unipotent radical, so we get (v), while (iii) is in Proposition 6.1. Finally, in cases (i) and (j), by Example 4.4 there is a dense pair, which lies inside a Borel subgroup, and (iii) was shown in Proposition 6.2. If $G=D_{4} \cdot \mathfrak{S}_{3}$, we apply Proposition 7.4 and Lemma 7.5.

The same result holds for $G$-classes as well (but we can include (vii) without any restrictions as all the cosets of outer involutions are conjugate).

Note that all in cases we see from the above arguments that $G$ either has infinitely many orbits or at most 3 orbits on $C_{1} \times C_{2}$. This was shown in the connected case in [7] aside from two cases where a bound of 4 was given. We show that 3 is an upper bound in these cases as well in the next two results.

Proposition 6.5. Let $k$ be an algebraically closed field of characteristic 2. Let $G=$ $\operatorname{Sp}_{2 n}(k)=\operatorname{Sp}(V)$. Let $x \in G$ be an involution with $(x v, v)=0$ for all $v \in V$. Then $C_{G}(x)$ has at most 3 orbits on the nonzero vectors of $V$.

Proof. Note that $W:=[x, V]$ is a totally singular $m$-space for some $m \leq n$. Let $X=$ $[x, V]^{\perp}$. Let $P$ be the stabilizer of $W$. So $P=Q L$ is a maximal parabolic subgroup of $G$ with unipotent radical $Q$ and Levi subgroup $L \cong \mathrm{GL}(W) \times \operatorname{Sp}(X / W)$. Note that $x \in Z(Q)$ and so we see that $C_{G}(x)=Q J$ where $J \cong \operatorname{Sp}(W) \times \operatorname{Sp}(X / W)$. The result now follows easily by noting that $J$ acts transitively on the nonzero elements of $W, X / W$ and $V / X$ and that if $v \in X \backslash W$, then $Q v=v+W$ and if $v \in V \backslash X$, then $Q v=v+X$ (if $W \neq X$, the three orbits are $W \backslash\{0\}, X \backslash W$ and $V \backslash X$ while if $W=X$, there are two orbits).

Proposition 6.6. Let $k$ be an algebraically closed field of characteristic $p \neq 2$. Let $G=\mathrm{SO}_{n}(k)=\mathrm{SO}(V)$. Let $x \in G$ be a unipotent element with all Jordan blocks of size at most 2. Then $C=C_{G}(x)$ has at most 3 orbits on nondegenerate 1-spaces of $V$.

Proof. First suppose that $n$ is a multiple of 4 and all Jordan blocks of $x$ have size 2. Then $W:=[x, V]=C_{V}(x)$ is a maximal totally singular subspace and $C=Q \operatorname{Sp}(W)$ where $Q$ is the unipotent radical of the stabilizer of $W$. Then $C$ is transitive on all cosets $v+W$ with $v$ outside $W$ and $Q$ is transitive on all vectors in $v+W$ of a given norm, whence we see that $C$ has a single orbit on nondegenerate 1-spaces (this is also clear from the proof of Proposition 6.1). Thus, we see that $C$ has two orbits on nonzero singular vectors (they are either in $W$ or not).

In the general case, we can write $V=V_{0} \perp W$ where $V_{0}$ is a nondegenerate space with $x$ trivial on $V_{0}$ and all Jordan blocks of $x$ have size 2 on $W$. Let $v$ be a nonsingular vector in $V$ and write $v=v_{0}+w$ with $v_{0} \in V$ and $w \in W$. By the argument in the first paragraph, we can find $x$-invariant nondegenerate subspaces $V_{1} \subset V_{0}$ and $W_{1} \subset W$ with $\operatorname{dim} V_{1} \leq 2$ and $\operatorname{dim} W_{1} \leq 4$ and $c \in C$ with $c v \in V_{1}+W_{1}$. Thus, we may assume that $n \leq 6$ (and clearly $n \geq 4$ ). It is straightforward to check the result in these cases.

This completes the proof of Corollary 1.2 . 


\section{Cosets OF ORDER 2 IN $D_{4}(k) \cdot \mathfrak{S}_{3}$}

Here, we discuss the situation left open in conclusion $(\mathrm{k})$ of Theorem 4.6 ,

Let $k$ be an algebraically closed field of characteristic $p \geq 0$ and $G=D_{4}(k) \cdot \mathfrak{S}_{3}$ (of adjoint type), the extension of a simple algebraic group of type $D_{4}$ by the full group of graph automorphisms $\mathfrak{S}_{3}$. Let $x, y \in G$ be outer involutions in two different cosets of $G^{\circ}$. Moreover, we assume that $x$ and $y$ correspond to reflections in some projective 8-dimensional representation of $\left\langle G^{\circ}, x\right\rangle$, resp. $\left\langle G^{\circ}, y\right\rangle$. Note that $G^{\circ}=C_{G^{\circ}}(x) C_{G^{\circ}}(y)$ whence $G^{\circ}$ is transitive on such pairs. Set $z=x y$. Thus $z$ is a graph automorphism of $G^{\circ}$ of order 3 with centralizer $C_{G^{\circ}}(z) \cong G_{2}(k)$.

We start with some examples. In all of these $C$ denotes the $G^{\circ}$-class of $x$ and $D$ will be a class in the coset $G^{\circ} y$. For convenience we recall the outer unipotent conjugacy classes of $\mathrm{GO}_{8}(k)$, with $k$ algebraically closed of characteristic 2 (see [15, p.236] and [10, Tab. 10], and the unipotent classes of $G_{2}(k)$ (see [9, Tab. B, Tab. 1]).

TABLE 1. Outer unipotent classes of $\mathrm{GO}_{8}(k), \operatorname{char}(k)=2$

\begin{tabular}{|l|cccc|}
\hline$x$ & $C(x) / R_{u}(C(x))$ & $\operatorname{dim} R_{u}(C(x))$ & $\operatorname{dim} C(x)$ & $A(u)$ \\
\hline $2.1^{6}$ & $C_{3}$ & 0 & 21 & 1 \\
$2^{3} .1^{2}$ & $A_{1}^{2}$ & 7 & 13 & 1 \\
$4.1^{4}$ & $A_{1}^{2}$ & 5 & 11 & $Z_{2}$ \\
$3^{2} .2$ & $A_{1}$ & 6 & 9 & 1 \\
$4.2^{2}$ & $A_{1}$ & 6 & 9 & 1 \\
$4.2^{2}$ & 1 & 7 & 7 & 1 \\
$6.1^{2}$ & $T_{1}$ & 4 & 5 & $Z_{2}$ \\
8 & 1 & 3 & 3 & 1 \\
\hline
\end{tabular}

TABLE 2. Unipotent classes of $G_{2}(k), \operatorname{char}(k) \neq 3$

\begin{tabular}{|l|cccc|rr|}
\hline$x$ & $C(x) / R_{u}(C(x))$ & $\operatorname{dim} R_{u}(C(x))$ & $\operatorname{dim} C(x)$ & $A(u)$ & \multicolumn{2}{|c|}{ in $D_{4}(k)$} \\
& & & & & $(p>3)$ & $(p=2)$ \\
\hline 1 & $G_{2}$ & 0 & 14 & 1 & $1^{8}$ & $1^{8}$ \\
$A_{1}$ & $A_{1}$ & 5 & 8 & 1 & $2^{2} .1^{4}$ & $2^{2} \cdot 1^{4}$ \\
$\tilde{A}_{1}$ & $A_{1}$ & 3 & 6 & 1 & $3.2^{2} .1$ & $2^{4}$ \\
$G_{2}\left(a_{1}\right)$ & 1 & 4 & 4 & $\mathfrak{S}_{3}$ & $3^{2} .1^{2}$ & $3^{2} .1^{2}$ \\
$G_{2}$ & 1 & 2 & 2 & $Z_{(2, p)}$ & 7.1 & 6.2 \\
\hline
\end{tabular}

Example 7.1. Let $D$ consist of elements conjugate to $y s=s y$ where $s \in C_{G^{\circ}}(y)$ is a semisimple element with at most 2 non-trivial eigenvalues. First assume that $s$ has a non-trivial eigenvalue $a$ of order prime to 6 . Note that $\operatorname{dim} C=7$ and that $\operatorname{dim} D=$ 17. Also note that we can arrange that $x y$ is conjugate to $z t$ where $t \in C_{G^{\circ}}(z)$ with $t$ semisimple and of order prime to 6 . Thus $\operatorname{dim} C_{G^{\circ}}(z t)=\operatorname{dim} C_{G_{2}(k)}\left(t^{3}\right) \leq 4$ and it follows 
TABLE 3. Unipotent classes of $G_{2}(k), \operatorname{char}(k)=3$

\begin{tabular}{|l|cccc|r|}
\hline$x$ & $C(x) / R_{u}(C(x))$ & $\operatorname{dim} R_{u}(C(x))$ & $\operatorname{dim} C(x)$ & $A(u)$ & in $D_{4}(k)$ \\
\hline 1 & $G_{2}$ & 0 & 14 & 1 & $1^{8}$ \\
$A_{1}$ & $A_{1}$ & 5 & 8 & 1 & $2^{2} .1^{4}$ \\
$\tilde{A}_{1}$ & $A_{1}$ & 5 & 8 & 1 & $3.2^{2} .1$ \\
$\tilde{A}_{1}^{(3)}$ & 1 & 6 & 6 & 1 & $3.2^{2} .1$ \\
$G_{2}\left(a_{1}\right)$ & 1 & 4 & 4 & $Z_{2}$ & $3^{2} .1^{2}$ \\
$G_{2}$ & 1 & 2 & 2 & $Z_{3}$ & 7.1 \\
\hline
\end{tabular}

that $\operatorname{dim}(x y)^{G^{\circ}} \geq 24$, whence we have equality. Thus, $C D=(x y)^{G^{\circ}}$ (since the right hand side is closed). Indeed the same argument shows that for any $\left(x^{\prime}, y^{\prime}\right) \in C \times D$, $\operatorname{dim}\left(C_{G^{\circ}}\left(x^{\prime}\right) \cap C_{G^{\circ}}\left(y^{\prime}\right)\right) \leq \operatorname{dim} C_{G^{\circ}}(x y)=4$, so $G^{\circ}=C_{G^{\circ}}(x) C_{G^{\circ}}(y)$. If $a \in k^{\times}$is arbitrary, the centralizer will only get bigger whence we still have the same factorization.

Example 7.2. Let $p \neq 2$ and $D$ consist of elements conjugate to $y u=u y$ where $u \in$ $C_{G^{\circ}}(y)$ is unipotent with Jordan block lengths $2^{2} .1^{4}$ or $3.1^{5}$.

First consider the case that $u$ is a long root element. Note that every $G^{\circ}$-orbit in $C \times D$ contains a representative $(x, y u)$ where $u$ is a long root element commuting with $y$ (since $G^{\circ}$ is transitive on $\left.x^{G^{\circ}} \times y^{G^{\circ}}\right)$. By Proposition 6.2, $C_{G^{\circ}}(z)$ has five orbits on long root elements in $G$. It is easy to see that only 2 of these orbits are contained in $C_{G}(y)$. Thus, $G^{\circ}$ has exactly 2 orbits on $C \times D$ (depending upon whether $u$ centralizes $z$ or not).

There is a subgroup $A_{1}(k)^{4}$ of $G^{\circ}$ normalized by $x, y$. We may assume that $y$ permutes the last two copies and $x$ permutes the middle two copies (so both centralize the first copy). If we take $u=\left(a_{1}, a_{2}, 1,1\right)$ where $a_{i}$ are nontrivial unipotent elements of $A_{1}(k)$, then $u$ has a single Jordan block of size 3 but $z u$ is conjugate to $z v=v z$ where $v$ has two Jordan blocks of size 3 (and two trivial Jordan blocks). It follows that $v$ lies in the conjugacy class $G_{2}\left(a_{1}\right)$ in $C_{G^{\circ}}(z)$. Thus, $\operatorname{dim}(z v)^{G^{\circ}}=24$ and so $G^{\circ}$ has a dense orbit on $C \times D$ (consisting of those pairs whose product is in the class of $z v$ ).

Example 7.3. Let $p=2$ and $D$ consist of 2-elements with Jordan block lengths $2^{3} \cdot 1^{2}$, $4.1^{4}$ or $3^{2} .2$ in the natural 8-dimensional representation of $\mathrm{GO}_{8}(k)$. An explicit calculation in the permutation representation of $\mathrm{SO}_{8}^{+}(2) \cdot \mathfrak{S}_{3}$ of degree 3510 (as maximal subgroup of $F i_{22}$ ) shows that the respective products contain elements of the form $z u$, with $z=x y$ a graph automorphism of order 3 and $u \in C_{G^{\circ}}(z) \cong G_{2}(k)$ unipotent in the $G_{2}(k)$-class $\tilde{A}_{1}, G_{2}\left(a_{1}\right), G_{2}$ respectively (see Table 2). Thus the corresponding classes $E=[z u]$ have dimensions 22,24, 26 respectively. As $\operatorname{dim} C+\operatorname{dim} D$ equals $7+15,7+17,7+19$ in the three cases, we conclude by Lemma 3.1 that $E$ is dense in $C D$ and that $C D$ consists of finitely many $G^{\circ}$ classes.

We now show that the above are the only examples:

Proposition 7.4. Let $C, D$ be $G^{\circ}$-orbits in $x G^{\circ}$ and $y G^{\circ}$. Then $C D$ is a finite union of $G^{\circ}$-orbits if and only if (after reordering if necessary) $C$ consists of involutions conjugate (in $G$ ) to $x$ and one of 
(1) $D$ consists of elements conjugate to $y t=t y$ where $t \in G^{\circ}$ is a semisimple element with at most 2 non-trivial eigenvalues;

(2) $p \neq 2, D$ consists of elements conjugate to $y u=u y$ where $u$ is unipotent with Jordan block lengths $2^{2} .1^{4}$ or $3.1^{5}$; or

(3) $p=2$ and $D$ consists of 2 -elements with Jordan block lengths $2^{3} .1^{2}, 4.1^{4}$ or $3^{2} .2$ in the natural 8-dimensional representation of $\mathrm{GO}_{8}(k)$.

Proof. We have already seen in Examples 7.17 .3 that all cases (1)-(3) really occur. So we need to show that these are the only possibilities with $C D$ containing only finitely many $G^{\circ}$-orbits. So assume that $C D$ consists of finitely many $G^{\circ}$-orbits or equivalently that all quasi-semisimple elements in the closure of $C D$ are conjugate.

First consider the case that $p \neq 2$ and $C$ and $D$ are both involutions but neither are reflections. Note that this prescribes $C$ and $D$ uniquely. Thus, for any pair of involutions $s, t \in C_{G^{\circ}}(z),(x s, y t) \in C \times D$ and $x s y t=z(s t)$. As $s, t$ run over all such involutions, st intersects infinitely many $G_{2}(k)$ classes (since we can take $s, t$ to be involutions in $G_{2}(k)$ ), whence $C D$ consists of infinitely many classes.

If $p=2$ and $C$ and $D$ both consist of 2-elements, then every unipotent class is represented in $\operatorname{Aut}\left(D_{4}(2)\right)$ and one checks directly using GAP that the answer is as stated in (3) of the assertion.

So next assume that $D$ does not consist of involutions and that $C$ and $D$ are both quasisemisimple classes. Then we choose $\left(x^{\prime}, y^{\prime}\right) \in C \times D$ which normalize a parabolic subgroup $P=Q L$ with $P$ the normalizer of a long root subgroup, $L$ is a Levi subgroup and $Q$ the unipotent radical of $P$. Note that $L=T A_{1}(k)^{3}$ where $T$ is a central 1-dimensional torus of $L$. We can assume that $x$ permutes the first two copies of $A_{1}(k)$ and $y$ the last two.

Since $C$ and $D$ consist of quasi-semisimple elements, we can choose $x^{\prime}, y^{\prime}$ actually normalizing $L$. It is straightforward to see that (aside from the cases allowed in the conclusion) $(C \cap L)(D \cap L)$ intersects infinitely many classes of $L . \mathfrak{S}_{3}$ whence $C D$ contains infinitely many $G^{\circ}$-orbits by Proposition 3.6 .

Thus, we may assume that all quasi-semisimple elements in the closure of $x^{\prime}$ are conjugate to $x$ and that the quasi-semisimple elements in the closure of $y^{\prime}$ are as given in (1) or (2). Moreover, at least one of $x^{\prime}, y^{\prime}$ is not quasi-semisimple.

Case 1. $x^{\prime}$ is quasi-semisimple and $y$ does not lie in the closure of $y^{\prime}$.

By passing to closures, we may assume that $y^{\prime}=y_{1} y=y y_{1}$ where $y_{1}=u t$ with $t$ semisimple having precisely two nontrivial eigenvalues and $u$ is a long root element. Note that such a class has a representative in $L$ and one easily checks that there are infinitely many classes represented there.

Case 2. $x^{\prime}$ is quasi-semisimple and $y$ lies in the closure of $y^{\prime}$.

In this case, we can assume that $p \neq 2$ (because of the remarks above about pairs of 2-elements). By passing to closures (and noting that we may assume the unipotent part of $y^{\prime}$ is not a long root element nor has just one single Jordan block of size 3), we may assume that $y^{\prime}=y u$ where $u$ has a single Jordan block of size 3 and two Jordan blocks of size 2. Again, we can see such an element in $L$ and obtain a contradiction.

Case 3. Neither $x^{\prime}$ nor $y^{\prime}$ are quasi-semisimple.

Passing to closures, we may assume that the unipotent parts of $x^{\prime}$ and $y^{\prime}$ are long root elements and again we can see such elements in $L$ and derive a contradiction. 
By symmetry, we have covered all cases and proved the result.

Lemma 7.5. In the situation of Proposition 7.4. $G^{\circ}$ acts transitively on $C \times D$ in case (1); it has 2 orbits in both situations of case (2); and there are 2, 3, respectively 2 orbits in case (3). Moreover, every $(c, d) \in C \times D$ normalizes a Borel subgroup of $G^{\circ}$.

Proof. The case (1) was already argued in Example 7.1, and similarly for the first pair of classes in case (2), in Example 7.2, If $u$ has Jordan blocks of lengths $3.1^{5}$ in case (2), we need to count orbits of $G_{2}(k)$ on the class of $u$ in $\mathrm{SO}_{7}(k)$.

Note that if $u \in \mathrm{SO}_{7}(k)$ is such a unipotent element, it has a unique 1-dimensional invariant (singular) subspace (namely the image of $(u-1)^{2}$ ) and then $u$ lies in the unipotent radical of the stabilizer $P_{1}$ of that subspace (corresponding to nonsingular vectors in the unipotent radical).

Note that $G_{2}(k)$ acts transitively on singular 1-spaces (this is well known, but note that in the 7-dimensional representation, the stabilizer of a highest weight space is a maximal parabolic subgroup $P$ of codimension 5 , thus $G_{2} / P$ is a 5-dimensional projective variety contained in $\mathrm{SO}_{7}(k) / P_{1}$ which is also projective of dimension 5 , so they are equal). So we can just count orbits of $P$ on the unipotent radical $Q$ of $P_{1}$. Now $Q$ is 5-dimensional and abelian and $P_{1} / Q$ acts on this as $T \mathrm{SO}_{5}(k)$, and $P /(Q \cap P)=T A_{1} U_{3}$ acts on this so that $Q=2 / 1 / 2$ as a module for $P$. Because of the torus it is enough to count orbits on nondegenerate 1-spaces, whence it is easy to see that $P^{\prime}$ has two such orbits acting on $Q$ (namely those spaces in the orthogonal complement of the 2 and those outside).

Finally, in case (3), first assume that $D$ contains unipotent elements with Jordan type $2^{3} \cdot 1^{2}$. As we saw in Example 7.3, one orbit consists of pairs $(c, d)$ such that $c d$ lies in class $\tilde{A}_{1}$ of a subgroup $G_{2}(k)$, with connected centralizer of dimension 6 . A second orbit is given by pairs $(c, d)$ with product in class $A_{1}$ in $G_{2}(k)$, and connected centralizer of dimension 8. Adding up the lengths of these two orbits over $\mathbb{F}_{2^{f}}$ shows that there no other orbits.

Next, assume that $D$ contains unipotent elements with Jordan type $4.1^{4}$. Any $d \in D$ can be written as $y w$ where $y$ is a reflection and $w$ is a long root element in $G^{\circ}$ (not centralizing $y$ for sure). This is not unique, but given any $(c, d) \in C \times D$, we can conjugate and assume $c=x$ and $d=y w$ as above. Now $G_{2}(k)$ centralizes $x$ and $y$ and so the number of orbits of $G$ on $C \times D$ is at most the number of orbits of $G_{2}(k)$ on the set of long root elements $w$ such that $y w$ has Jordan type $4.1^{4}$.

Since $G_{2}(k)$ has 5 orbits on long root elements and at least two of them do not have the property that $y w$ has order 4 (namely the ones in $G_{2}(k)$ and also the ones in $\operatorname{Sp}_{6}(k)$ ), there are at most 3 orbits. In fact, we claim that there are 3 orbits. Two of the orbits are interchanged by $y$ and we can see such a $w$ in $A_{1}(k) \times A_{1}(k)$ where $y$ is interchanging the 2 factors and $w$ is trivial in one of the factors. Clearly $y w$ has order 4 and has a 5-dimensional fixed spaces, so $y w$ has the correct Jordan type. Since $y$ interchanges those two orbits, we see that there are at least 2 orbits. However, neither of these is the dense orbit (e.g., they have the same dimension) and so there must be a third orbit (alternatively, one can compute over $\mathbb{F}_{2}$ ).

Finally consider the case that $D$ consists of unipotent elements with Jordan type $3^{2} .2$. 
Computing over $\mathbb{F}_{2}$, we see that $C D$ contains the conjugacy classes of elements $z u=u z$ where $z$ is a graph automorphism of order 3 with centralizer $G_{2}(k)$ and $u$ is either a regular unipotent element of $G_{2}(k)$ or is in the conjugacy class $G_{2}\left(a_{1}\right)$.

First consider a pair $(c, d) \in C \times D$ with $c d=z u=u z$ where $u$ is a regular unipotent element in $G_{2}(k)$. It follows that $J$, the centralizer of $\langle c, d\rangle$ is contained in the centralizer of $z u$ which has connected component a 2-dimensional unipotent group and has two components. Since $\operatorname{dim} C+\operatorname{dim} D=26$, it follows that $\operatorname{dim} J \geq 2$, whence $J^{\circ}$ is a 2dimensional unipotent group. Moreover, this must correspond to the dense orbit of $G^{\circ}$ and so it is unique. Computing such triples over $\mathbb{F}_{2}$, we see that there are two orbits each of size $\left|G^{\circ}(2)\right| / 4 / 2$. It follows by Lang's theorem that $\left[J: J^{\circ}\right]=2$ and so there is a single $G^{\circ}$-orbit splitting into two $G(q)$-orbits each of size $(1 / 2)\left|G^{\circ}(q)\right| / q^{2}$.

It follows by counting that the dimension of the complement of the dense orbit in $C \times D$ is 24 and moreover, there is at most 1 orbit of that dimension. Let $H$ be the centralizer of a $(c, d) \in C \times D$ with product $z u=u z$ where $u \in G_{2}\left(a_{1}\right)$. The centralizer $H$ of $z u$ in $G^{\circ}$ has connected component a 4-dimensional unipotent group with $H / H^{\circ} \cong \mathfrak{S}_{3}$ (see Table 2). We see that over $\mathbb{F}_{2}$ this orbit breaks up into three orbits of sizes $e\left|G^{\circ}(2)\right| / 2^{4}$ withe $e=1 / 2,1 / 3$ and $1 / 6$. It follows that $H$ is the centralizer of $\langle c, d\rangle$ and this orbit breaks up into 3 orbits over $\mathbb{F}_{q}$ of sizes $e\left|G^{\circ}(q)\right| / q^{4}$ with $e=1 / 2,1 / 3$ and $1 / 6$. Thus, the number of $\mathbb{F}_{q}$ points in this orbit of the algebraic group is $\left|G^{\circ}(q)\right| / q^{4}$.

Since $|C(q)| D(q)|=| G^{\circ}(q)\left|/ q^{4}+\right| G^{\circ}(q) \mid / q^{2}$, it follows that there are no further orbits and so $G^{\circ}$ has exactly 2 orbits on $C \times D$.

The last assertion follows by noting that in the examples we produce $(c, d) \in C \times D$ in the dense orbit which normalize a Borel subgroup. Since normalizing a Borel subgroup is a closed condition (as $G / B$ is a projective variety), this implies the result.

We next consider commutators of $G^{\circ}$-orbits in our situation. Here, we have the following curious situation:

Example 7.6. As above let $x, y \in G=D_{4}(k) . \mathfrak{S}_{3}$ be quasi-central in distinct cosets of order 2 modulo $H:=G^{\circ}$. Then for any $g \in H$ we have

$$
[x, y g]=x(y g)^{-1} x y g=x g^{-1}(y x y) g \in x^{H} t^{H}=(x t)^{H}
$$

where $t=y x y$ is a quasi-central element (reflection) in the third such coset. So letting $C=$ $x^{H}$ and $D=y H$, we see that $[C, D]$ is a single $H$-orbit (namely the graph automorphisms of order 3 with centralizer $\left.G_{2}(k)\right)$. In particular, $G$ certainly need not have finitely many orbits on $C \times D$. Hence, the analogue of Theorem 6.4(vii) fails in this situation.

Proposition 7.7. Let $G=D_{4}(k) \cdot \mathfrak{S}_{3}$ and $C, D$ two $G^{\circ}$-orbits in distinct cosets of $G^{\circ}$ of order 2. Then $[C, D]$ is the union of finitely many $G^{\circ}$-orbits if and only if (up to order) $C$ consists of reflections.

Proof. We have seen in Example 7.6 that if $C$ consists of reflections and $D$ is any class, then $[C, D]=z^{G^{\circ}}$ where $z$ is a graph automorphism of order 3 with centralizer $G_{2}(k)$. Thus, by taking closures we may assume that one of the following holds:

(1) $C$ and $D$ are both quasi-semisimple;

(2) $C$ and $D$ are both reflections times long root elements; or

(3) $C$ is quasi-semisimple and $D$ is as in (2). 
We can see all these cases in $A_{1}^{4}$ and get a contradiction (assuming that neither $C$ nor $D$ consists of reflections).

Here is another corollary (which could be proved more directly):

Corollary 7.8. Let $G$ be an almost simple algebraic group. Suppose that $C_{1}, C_{2}, C_{3}$ are nontrivial $G^{\circ}$-orbits. Then $C_{1} C_{2} C_{3}$ consists of infinitely many $G^{\circ}$-orbits unless (up to order) $C_{1}$ and $C_{2}$ are reflections in different cosets modulo $G^{\circ}$ and $C_{3}$ consists of long root elements.

Proof. Observe that for $C_{1}$ and $C_{2}$ reflections in different cosets and $C_{3}$ long root elements, $C_{1} C_{2}=D:=z^{G^{\circ}}$ and so indeed $C_{1} C_{2} C_{3}=D C_{3}$ is a finite union of classes by Theorem 4.6.

In any case other than $D_{4}(k) \cdot \mathfrak{S}_{3}$ with an outer automorphism, two of the $C_{i}$ must either be inner or outer and so already $C_{i} C_{j}$ consists of infinitely many inner classes again by Theorem 4.6 (and by working in the normalizer of a torus, infinitely many classes times $C_{k}$ gives infinitely many classes).

If $G$ is connected, by passing to closures, we may assume that each $C_{i}$ is either semisimple or unipotent. We know that two semisimple elements do not work and so at least two of the classes must be unipotent. By taking closures, they can be taken to be root elements (and so for roots of distinct lengths). So we reduce to the rank 2 case, i.e. $B_{2}(k)$ (characteristic 2) or $G_{2}(k)$ (characteristic 3) by [7]. But then the product of the two unipotent classes contains a regular unipotent element and that is not one of our examples.

\section{INFINITE FIELDS}

Let's also note the following easy consequence of our results in [7]:

Corollary 8.1. If $G$ is a simple compact (real) Lie group and $C$ and $D$ are non-central conjugacy classes of $G$, then $C D$ is an infinite union of classes.

Proof. It follows easily from the fact that $G$ is Zariski dense in $\bar{G}$, the complexification of $G$, that any class is dense in its closure $\bar{C}$ in $\bar{G}$. Now if $C D$ is a finite union of classes, then taking closures shows that $\bar{C} \bar{D}$ is a finite union of $\bar{G}$-classes as well, and as $C$ and $D$ are semisimple classes, by [7, Thm. 1.1] this cannot happen.

For the disconnected case, we would get only the semisimple cases (e.g. in type $A$ ). The previous result extends to infinite fields with $G(K)$ an anisotropic simple group (i.e., containing no nontrivial unipotent elements).

The same ideas give the following result.

Corollary 8.2. Let $K$ be an infinite field and $G(K)$ some form of a simple algebraic group $G$ over $K$. Let $C \subset G(K)$ be a non-central conjugacy class. Then $C C$ is an infinite union of classes.

Proof. Let $\bar{C}$ denote the Zariski closure of $C$. Then the semisimple part of elements in $\bar{C}$ is unique, since in any rational faithful representation, elements in $\bar{C}$ have the same characteristic polynomial as those in $C$, as $G(K)$ is Zariski dense in $G$ (see e.g. [16, Cor. 13.3.9]). Thus, if $C C$ consists of finitely many classes, so does $\bar{C} \bar{C}$, which is not possible by [7, Thm. 1.1]. 


\section{REFERENCES}

[1] M. Aschbacher, G. Seitz, Involutions in Chevalley groups over fields of even order. Nagoya Math. J. 63 (1976), 1-91.

[2] F. Digne, J. Michel, Groupes réductifs non connexes. Ann. Sci. École Norm. Sup. (4) 27 (1994), $345-406$.

[3] D. Gorenstein, R. Lyons, R. Solomon, The Classification of the Finite Simple Groups. Number 3. Mathematical Surveys and Monographs, American Mathematical Society, Providence, RI, 1998.

[4] R. M. Guralnick, A note on pairs of matrices with rank one commutator. Linear and Multilinear Algebra 8 (1979/80), 97-99.

[5] R. M. Guralnick, Intersections of conjugacy classes and subgroups of algebraic groups. Proc. Amer. Math. Soc. 135 (2007), 689-693.

[6] R. M. Guralnick, G. Malle, Variations on the Baer-Suzuki theorem. In preparation.

[7] R. M. Guralnick, G. Malle, P. H. Tiep, Products of conjugacy classes in finite and algebraic simple groups. Advances Math. 234 (2013), 618-652.

[8] N. Katz, Rigid Local Systems. Annals of Mathematics Studies, 139. Princeton University Press, Princeton, NJ, 1996.

[9] R. Lawther, Jordan block sizes of unipotent elements in exceptional algebraic groups. Comm. Algebra 23 (1995), 4125-4156.

[10] G. Malle, Generalized Deligne-Lusztig characters. J. Algebra 159 (1993), 64-97.

[11] G. Malle, Green functions for groups of types $E_{6}$ and $F_{4}$ in characteristic 2. Comm. Algebra 21 (1993), 747-798.

[12] G. Prasad, Weakly-split spherical Tits systems in quasi-reductive groups. Amer. J. Math. (to appear), arXiv: 1103.5970 .

[13] R. W. Richardson, On orbits of algebraic groups and Lie groups. Bull. Austral. Math. Soc. 25 (1982), 1-28.

[14] T. ShoJI, The conjugacy classes of Chevalley groups of type $\left(F_{4}\right)$ over finite fields of characteristic $p \neq 2$. J. Fac. Sci. Univ. Tokyo Sect. IA Math. 21 (1974), 1-17.

[15] N. Spaltenstein, Classes Unipotentes et Sous-Groupes de Borel. Lecture Notes in Math., 946. Springer-Verlag, Berlin, 1982.

[16] T. A. Springer, Linear Algebraic Groups. Second edition. Progress in Mathematics, 9. Birkhäuser Boston, Boston, MA, 1998.

[17] R. Steinberg, Endomorphisms of Linear Algebraic Groups. Memoirs Amer. Math. Soc., 80, American Mathematical Society, Providence, RI, 1968.

Department of Mathematics, University of Southern California, Los Angeles, CA 90089-2532, USA

E-mail address: guralnic@usc.edu

FB Mathematik, TU Kaiserslautern, Postfach 3049, 67653 Kaiserslautern, Germany

E-mail address: malle@mathematik.uni-kl.de 


\title{
PRODUCTS AND COMMUTATORS OF CLASSES IN ALGEBRAIC GROUPS
}

\author{
ROBERT GURALNICK AND GUNTER MALLE
}

Dedicated to the memory of Tonny Springer

\begin{abstract}
We classify pairs of conjugacy classes in almost simple algebraic groups whose product consists of finitely many classes. This leads to several interesting families of examples which are related to a generalization of the Baer-Suzuki theorem for finite groups. We also answer a question of Pavel Shumyatsky on commutators of pairs of conjugacy classes in simple algebraic groups. It turns out that the resulting examples are exactly those for which the product also consists of only finitely many classes.
\end{abstract}

\section{INTRODUCTION}

The Baer-Suzuki theorem asserts that in a finite group $G$, if $x \in G$ is such that $\left\langle x, x^{g}\right\rangle$ is a $p$-group for all $g \in G$, then $\left\langle x^{G}\right\rangle$ is a normal $p$-subgroup of $G$.

We were recently informed by Bernd Fischer that Reinhold Baer had asked what one can say if, given $x, y \in G$, we have that $\left\langle x, y^{g}\right\rangle$ is a $p$-group for all $g \in G$. Examples in Guralnick-Malle-Tiep [7] show that there is not too much to say in general. However, with some extra hypothesis [7, 6], there are generalizations along these lines.

Here we consider the corresponding question for almost simple algebraic groups. Our main result is the following characterization, part of which is an analogue of the corresponding result in the connected case [7, Cor. 5.14]:

Theorem 1.1. Let $G$ be an almost simple algebraic group over an algebraically closed field, and let $C, D$ be $G^{\circ}$-classes. The following are equivalent:

(i) $C D$ is a finite union of $G^{\circ}$-conjugacy classes.

(ii) $C_{G^{\circ}}\left(x_{1}\right) \backslash G^{\circ} / C_{G^{\circ}}\left(x_{2}\right)$ is finite for all $\left(x_{1}, x_{2}\right) \in C \times D$.

(iii) $\left\langle x_{1}, x_{2}\right\rangle$ normalizes some Borel subgroup of $G^{\circ}$ for every $\left(x_{1}, x_{2}\right) \in C \times D$.

Moreover, any of the above conditions implies:

(iv) $[C, D]:=\{[x, y] \mid(x, y) \in C \times D\}$ is a finite union of $G^{\circ}$-conjugacy classes.

Here, an almost simple algebraic group is a possibly disconnected linear algebraic group whose connected component $G^{\circ}$ is simple and such that $C_{G}\left(G^{\circ}\right)=Z\left(G^{\circ}\right)$. See Theorem 6.4 for further equivalent conditions. Note that the result holds for $G$-classes as well

Date: October 31, 2018.

2010 Mathematics Subject Classification. Primary 20G07; Secondary 20E32, 20E45, 20F12.

Key words and phrases. conjugacy classes, commutators, algebraic groups.

The first author was partially supported by the NSF grant DMS-1001962 and the Simons Foundation Fellowship 224965. The second author gratefully acknowledges financial support by ERC Advanced Grant 291512. 
as long as we avoid the case $G=D_{4} \cdot \mathfrak{S}_{3}$ (because in the disconnected cases one of the classes is outer and so the centralizer intersects all cosets except in the excluded case).

While some implications in this statement have general proofs, others rely on our explicit classification of all pairs of classes with the stated properties.

Thus, we first classify in Theorem 3.7 all pairs $C, D$ of unipotent classes in (disconnected) almost simple algebraic groups such that every pair in $C \times D$ generates a unipotent group (the connected case was done in [7]). In fact, it turns out to be equivalent to the condition that $C D$ consists of unipotent elements.

We also extend the result of [7, Thm. 1.1] to the disconnected case by classifying pairs $C, D$ of conjugacy classes in (disconnected) almost simple groups such that $C D$ is a finite union of conjugacy classes.

It should be noted that for connected groups examples occur if and only if the group is of non-simply laced type, while for disconnected groups examples exist if and (clearly) only if the type is simply laced. See Proposition 4.12 for some relation between these two types of examples. Let's point out the following consequence of our classification: whenever $C D$ is a finite union of conjugacy classes, then at least one of $C, D$ contains a quasi-central class in its closure (recall that a quasi-central class is the smallest conjugacy in a given coset of $G^{\circ}$ ). It would be nice to have an a priori proof of this.

Two corollaries of the results and proofs are (extending the results of [7] to the disconnected case):

Corollary 1.2. Let $G$ be an almost simple algebraic group over an algebraically closed field. Suppose that $C$ and $D$ are conjugacy classes of $G$. Then either $C D$ is the union of at most 3 conjugacy classes or $C D$ contains infinitely many conjugacy classes.

In the previous result, if we replace $G$-classes by $G^{\circ}$-classes, then 5 classes suffice.

Corollary 1.3. Let $G$ be an almost simple algebraic group over an algebraically closed field $k$. If $x \in G \backslash Z\left(G^{\circ}\right)$, then $\operatorname{dim}\left(C_{G}(x) y C_{G}(x)\right)<\operatorname{dim} G$ for all $y \in G$, unless $G=D_{4}(k) . \mathfrak{S}_{3}$ and $x$ is an outer involution with $C_{G}(x)=B_{3}(k)$ and $y$ is not in $\left\langle G^{\circ}, x\right\rangle$.

Note that in the excluded case, we really get counter-examples. This last corollary in the connected case was used by Prasad 12 in his study of quasi-reductive groups.

We also answer in the case of algebraic groups a question that Pavel Shumyatsky had asked in the finite case:

Theorem 1.4. Let $G$ be an almost simple algebraic group over an algebraically closed field of characteristic $p \geq 0$. Let $C$ be $a G^{\circ}$-conjugacy class of $G$ outside $Z\left(G^{\circ}\right)$. Then $[C, C]$ is the union of infinitely many conjugacy classes, where as before,

$$
[C, C]:=\{[x, y] \mid x, y \in C\} .
$$

It is worth noting that we also observe that an old result of the first author [4] answers a question of Nick Katz [8, 2.16.7] about commutators being transvections. This is useful in the proof of the algebraic group result.

Finally, we observe that it is quite easy to extend our results to almost simple algebraic groups over infinite fields. In particular, we can answer the following question of Diaconis. 
Theorem 1.5. Let $G$ be a simple compact Lie group. If $C$ and $D$ are noncentral conjugacy classes of $G$, then $C D$ consists of infinitely many conjugacy classes.

The paper is organized as follows. In Section 2 we collect some auxiliary results on closed conjugacy classes in not necessarily connected algebraic groups. We then consider products of unipotent classes in disconnected algebraic groups in Section 3 and classify all cases where the product consists of finitely many classes in Theorem 3.7. In Section 4 we classify arbitrary products of classes meeting only finitely many conjugacy classes and thus prove Theorem 4.6. In Section 5 we show that these are precisely the pairs of conjugacy classes whose commutator consists just of finitely many classes, see Theorem 5.2. In Section 6, we prove the common characterization in Theorem 1.1 of various finiteness properties on products, commutators and double cosets and prove Corollary [1.2. In Section 17, we fully investigate the products of two non-trivial cosets of order 2 in the disconnected groups of type $D_{4}(k) \cdot \mathfrak{S}_{3}$. In the final section, we point out how we may extend our results to the case of infinite fields.

The similar questions for finite groups are considerably harder and will be dealt with in a forthcoming paper [6].

We thank Pavel Shumyatsky for communicating his question to us, George McNinch for pointing out the relevance of the results of Richardson [13], and Sebastian Herpel for some comments on a preliminary version.

\section{Closed CONJugacy Classes in Disconnected Algebraic Groups}

We'll need some information on closed conjugacy classes. In connected reductive algebraic groups, the closed classes are precisely the semisimple ones. In the disconnected case, there can be non-semisimple elements whose class is closed. Following Steinberg [17, $\S 9]$ we call an automorphism of a connected reductive algebraic group quasi-semisimple if it normalizes a Borel subgroup and a maximal torus thereof. We also use the following result of Spaltenstein [15, II.2.21]:

Theorem 2.1 (Spaltenstein). Let $G$ be a reductive algebraic group over an algebraically closed field. Then any coset of $G^{\circ}$ in $G$ contains at most one closed unipotent class. In particular, this class is contained in the closure of all unipotent classes in that coset.

Lemma 2.2. Let $H$ be a simple algebraic group over an algebraically closed field of characteristic $p>0$. Assume that $u$ is a quasi-semisimple automorphism of $H$ of order $p$ and $s \in H$ is a semisimple element commuting with $u$. Then us is also quasi-semisimple.

Proof. By assumption $u$ normalizes a Borel subgroup $B$ of $H$ and a maximal torus $T \leq B$. Let $D:=C_{H}(u)$. By [2, Thm. 1.8(iii)], $T \cap D$ is a maximal torus of $D, B \cap D$ is a Borel subgroup of $D$ and $D$ is connected (see also Steinberg [17, 8.2] for the simply connected case). (Alternatively, this can be seen by inspection of the various cases.) Thus, $s$ is conjugate in $D$ to an element of $T \cap D$ and so we may assume that $s \in T \cap D$. Then us normalizes $T$ and $B$.

Theorem 2.3. Let $G$ be a reductive algebraic group over an algebraically closed field $k$ of characteristic $p$ and let $C \subset G$ be a conjugacy class, $g \in C$. Then the following are equivalent: 
(i) $C$ is closed;

(ii) $C_{G}(g)$ is reductive; and

(iii) $g$ is quasi-semisimple.

Proof. The equivalence of (i) and (iii) is shown in [15, Lemme II.1.15] (see also [15, Cor. II.2.22]).

Now assume (i), so $C$ is closed. Then $C$ is an affine variety (since $G$ is affine). It follows by [13, Lemma 10.1.3] that $C_{G}(g)$ is reductive.

Finally let's show that (ii) implies (iii). Note that all the conclusions concern only $\left\langle g, G^{\circ}\right\rangle$ and so we assume that $G=\left\langle g, G^{\circ}\right\rangle$. There is no loss in assuming that $G^{\circ}$ is semisimple with $C_{G}\left(G^{\circ}\right)=1$. Moreover, we may assume that $g$ acts transitively on the simple components of $G^{\circ}$. If there are $m$ components, then $C_{G^{\circ}}(g) \cong C_{L}\left(g^{m}\right)$ where the components are isomorphic to $L$. Moreover, $g$ is quasi-semisimple if and only if $g^{m}$ is. Thus, it suffices to assume that $G^{\circ}$ is simple.

Assume that $g$ is not quasi-semisimple. We show that $C_{G}(g)$ is not reductive.

Write $g=s u=u s$ where $s$ is semisimple and $u$ is unipotent. Let $v$ be a generator of $\langle u\rangle \cap G^{\circ}$. If $v \neq 1$, then by the Borel-Tits theorem, the unipotent radical $U$ of $C_{G^{\circ}}(s v)$ is nontrivial, whence since $u$ acts as a $p$-element on $U, C_{U}(u)^{\circ}$ is a positive dimensional subgroup contained in the unipotent radical of $C_{G}(g)$. So $v=1$, and hence $u^{p}=1$.

If $p \neq\left[G: G^{\circ}\right]$, then $g=s$ is semisimple and in particular quasi-semisimple. Thus we may assume that $p=\left[G: G^{\circ}\right]$ (in particular, $p=2$ or 3 ).

By the discussion above, we have that $u$ is an automorphism of order $p$ and $s \in G^{\circ}$. Note that $u$ does not lie in the unique class of outer unipotent elements which are quasisemisimple, since otherwise by the previous lemma, $g$ would also be quasi-semisimple.

We consider the various cases. For the moment, exclude the case that $p=2$ and $G^{\circ}=D_{n}(k), n \geq 4$. If $p=2$, it follows by [1, 19.7-19.9] that $u=v t$ where $v$ is a quasi-semisimple graph automorphism and $t$ is a long root element in $C_{G^{\circ}}(v)$. But then, $C_{G^{\circ}}(u)<C_{G^{\circ}}(v)$ has a nontrivial unipotent radical. The same argument applies for $p=3$ and $G=D_{4}(k)$ [3, 4.9.2].

Finally, consider the case that $G^{\circ}=D_{n}(k), n \geq 4$ and $p=2$. Let $V$ be the natural $2 n$-dimensional orthogonal module for $G$.

Suppose that $s=1$. Note that $u$ is not a transvection on $V$ (which is the unique quasisemisimple unipotent class in $u G^{\circ}$ ). Thus, the fixed space $W$ of $u$ has dimension at most $2 n-2$. Let $R$ be the radical of $W$ (with respect to the alternating form preserved by $G$ ). Note that $R \neq 0$ for otherwise, $W$ is nonsingular and so $V=W \oplus W^{\perp}$, a contradiction since $u$ will have fixed points on $W^{\perp}$. If $R$ is totally singular with respect to the quadratic form preserved by $G$, then the centralizer of $u$ is contained in the parabolic subgroup stabilizing $R$ and so $C_{Q}(u)$ is a positive dimensional subgroup of the unipotent radical of $C_{G^{\circ}}(u)$, which thus is not reductive. Indeed the same argument applies if $\operatorname{dim} R>1$, for then replace $R$ by $R^{\prime}$, the radical with respect to the quadratic form. So $R$ is 1-dimensional and is generated by a non-isotropic vector. The stabilizer of this 1-dimensional space is $\langle x\rangle \times \operatorname{Sp}_{2 n-2}(k)$ with $x$ a transvection. If $u \notin\langle x\rangle$, then $C_{G^{\circ}}(u) \cong C_{\operatorname{Sp}_{2 n-2}(k)}(y)$ for some nontrivial unipotent element $y$ and so is not reductive.

If $s \neq 1$, write $V=W \perp W^{\prime}$ where $W=[s, V]$. Note that $W \neq 0$ since $s \neq 1$. Also, (since $p=2$ ) the centralizer of $s$ in the stabilizer of $W$ is contained in $\operatorname{SO}(W)$. In particular, if $u$ acts nontrivially on $W$, it follows (by inspection or Borel-Tits) that the 
centralizer of $G$ has non-trivial unipotent radical on $W$ and so on $V$. Thus, $u$ is trivial on $W$. Since the centralizer of $u$ on $W^{\prime}$ is reductive, it follows by the previous case that $u$ induces a transvection on $W^{\prime}$, whence $u$ is a transvection. This contradiction completes the proof.

Note that the proof actually also shows that:

Corollary 2.4. Let $G$ be a reductive algebraic group over an algebraically closed field. Let $g \in G$ with semisimple part s. There exists a unique closed conjugacy class in $g G^{\circ}$ whose elements have semisimple part conjugate to $s$.

We also have the obvious corollary.

Corollary 2.5. Let $G$ be a reductive algebraic group over an algebraically closed field $k$. Assume that the order of $g$ in $G / G^{\circ}$ is prime to the characteristic of $k$. Then the class of $g$ is closed if and only if $g$ is semisimple.

Proof. In this situation, quasi-semisimple elements are semisimple (see [17, §9]), and the result follows.

\section{Pairs of unipotent Classes in almost simple Algebraic Groups}

In this section we classify pairs of unipotent conjugacy classes in almost simple algebraic groups such that all pairs of elements generate a unipotent subgroup (see Theorem 3.7). Note that the connected case was already treated in [7, Thm. 1.1]. First note the following:

Lemma 3.1. Let $G$ be a reductive algebraic group and $C_{1}, C_{2}, D \subset G$ three $G^{\circ}$-orbits under conjugation. Assume that $D \subseteq C_{1} C_{2}$ and $\operatorname{dim} C_{1}+\operatorname{dim} C_{2}=\operatorname{dim} D$. Then:

(a) $D$ is open dense in the product $C_{1} C_{2}$;

(b) $\left\{\left(c_{1}, c_{2}\right) \in C_{1} \times C_{2} \mid c_{1} c_{2} \in D\right\}$ is a dense open subset of $C_{1} \times C_{2}$;

(c) $C_{1} C_{2}$ consists of finitely many classes.

Proof. Since $G^{\circ}$ is connected, the $G^{\circ}$-orbits $C_{1}, C_{2}, D$ are irreducible and then so is $C_{1} C_{2}$. Now $\operatorname{dim} C_{1} C_{2} \leq \operatorname{dim} C_{1}+\operatorname{dim} C_{2}=\operatorname{dim} D$ and $D \subset C_{1} C_{2}$ imply that $\operatorname{dim} C_{1} C_{2}=\operatorname{dim} D$. Thus, their closures agree. Since any two dense subsets intersect non-trivially, $D$ is the unique dense class in $C_{1} C_{2}$, so it must be open. The second statement now follows. As the closure of any class consists of finitely many classes, we have (c).

We now collect some actual examples:

Example 3.2. Let $S=\mathrm{SL}_{2 n}(k), n \geq 2$, with $k$ an algebraically closed field. Let $x \in S$ be a transvection, $y \in \operatorname{Aut}(S)$ a graph automorphism with centralizer $\operatorname{Sp}_{2 n}(k)$ and $G=\langle S, y\rangle$. Let $U$ be a root subgroup containing $x$. Let $N=N_{S}(U)$. Since $\operatorname{Sp}_{2 n}(k)$ has two orbits on pairs consisting of a 1-space and a hyperplane containing it we see that $\left|\operatorname{Sp}_{2 n}(k) \backslash S / N\right|=2$. Thus, $x^{S} \times y^{S}$ consists of two $S$-orbits. One orbit is the set of commuting pairs. When $k$ has characteristic $p>0$, reducing to the case of $n=2$ shows that in the other orbit the product will have order $2 p$. In particular, if $p=2,\left\langle x, y^{g}\right\rangle$ is either elementary abelian of order 4 or a dihedral group of order 8 for all $g \in S$. 
Example 3.3. Let $G=\mathrm{GO}_{2 n}(k), n \geq 4$, with $k$ an algebraically closed field of characteristic 2. Then $G$ fixes a non-degenerate alternating form $($,$) on V=k^{2 n}$, so we may view $G$ as a subgroup of the isometry group $\operatorname{Sp}_{2 n}(k)$ of this form. Let $x \in G$ be a transvection, and $y$ an involution with $(y v, v)=0$ for all $v \in V$. Then by [7, Ex. 6.6], the order of $x y$ is either 2 or 4 , so $x, y$ always generate a 2 -group.

Example 3.4. Let $k$ be an algebraically closed field of characteristic 3 and $G=\mathrm{SO}_{8}(k) .3$, the extension of the simple algebraic group $G^{\circ}=\mathrm{SO}_{8}(k)$ by a graph automorphism of order 3. Let $C_{1}$ be the class of long root elements in $G^{\circ}$, with centralizer of type $3 A_{1}$, and $C_{2}$ the class of the graph automorphism $\sigma$ with centralizer $G_{2}(k)$ in $G^{\circ}$. We claim that $C_{1} C_{2}$ consists of 3 -elements.

Indeed, let $x_{i}(1), 1 \leq i \leq 4$, denote long root elements for the four simple roots, labelled so that the node in the centre of the Dynkin diagram has label 2, and $\sigma$ permutes $x_{1}(1), x_{3}(1), x_{4}(1)$ cyclically. So $x:=x_{2}(1) \in C_{1}$, and it's easily seen that $y:=$ $x_{1}(1) x_{3}(1) x_{4}(1) \sigma$ is conjugate to $\sigma$, so lies in $C_{2}$. The product $x y=x_{2}(1) x_{1}(1) x_{3}(1) x_{4}(1) \sigma$ lies in a class $D$, denoted $C_{4}$ in [15, I.3], of dimension 24 (see also the representative $u_{4}$ in [10, Tab. 8]). Now $C_{1}$ has dimension 10 and $C_{2}$ has dimension 14. Since $C_{1}$ is a single class under $G^{\circ}$-conjugation, and $C_{2}, D$, being outer classes, necessarily are, Lemma 3.1 shows that $D$ is dense in $C_{1} C_{2}$.

Since $\sigma$ normalizes the maximal unipotent subgroup generated by the standard positive root subgroups, $\langle x, y\rangle$ is unipotent for our choice of elements $x \in C_{1}, y \in C_{2}$ above, hence for all choices. Our claim follows.

Note that $x_{2}(1)$ and $x_{1}(1) x_{3}(1) x_{4}(1)$ are long, respectively short root elements in the centralizer $C_{G^{\circ}}(\sigma)=G_{2}(k)$ of $\sigma$, with product a regular unipotent element. This thus leads to the example in $G_{2}(k)$ from [7, Ex. 6.1], and we see that in both cases, the generated groups in the generic case agree (see also Proposition 4.12).

Example 3.5. Let $k$ be an algebraically closed field of characteristic 2 and $G=E_{6}(k) .2$, the extension of a simple algebraic group $G^{\circ}=E_{6}(k)$ of type $E_{6}$ by a graph automorphism of order 2. Let $C_{1}$ be the class of long root elements in $G^{\circ}$, with centralizer $U . A_{5}(k)$, where $U$ is unipotent of dimension 21, and $C_{2}$ the class of the graph automorphism $\sigma$ with centralizer $F_{4}(k)$ in $G^{\circ}$. We claim that again $C_{1} C_{2}$ only contains unipotent elements.

Indeed, let $x_{i}(1), 1 \leq i \leq 6$, denote long root elements for the six simple roots, labelled in the standard way (so that node 4 is at the centre of the Dynkin diagram, and $\sigma$ interchanges $\left.x_{3}(1), x_{5}(1)\right)$. So $x:=x_{4}(1) \in C_{1}$, and it's easily seen that $y:=x_{3}(1) x_{5}(1) \sigma$ is conjugate to $\sigma$, so lies in $C_{2}$. The product $x y=x_{4}(1) x_{3}(1) x_{5}(1) \sigma$ lies in a class $D$, with representative $u_{15}$ in [15, p. 160], of dimension 48 (see also [11, Tab. 10]). As $C_{1}$ has dimension 22 and $C_{2}$ has dimension 26, D is dense in $C_{1} C_{2}$ by Lemma 3.1. Again, $\sigma$ normalizes the maximal unipotent subgroup generated by the positive root subgroups, so $\langle x, y\rangle$ is unipotent, and hence by density this holds for all pairs from $C_{1} \times C_{2}$. (Note that both classes have representatives in a Levi subgroup of type $A_{3} .2$, where they correspond to Example 3.2.)

As in the case of $\mathrm{SO}_{8}(k)$, we also recover the example in the connected group $F_{4}(k)$ in characteristic 2 from [7, Ex. 6.3], by considering the centralizer $C_{G^{\circ}}(\sigma)=F_{4}(k)$ of $\sigma$. There, $x_{4}(1)$ is a long root element, and $x_{3}(1) x_{5}(1)$ is a short root element. We see that the group generated by them is isomorphic to the one in $G$. 
We are now ready to prove the main result of this section. Throughout our proofs the following result will make inductive arguments work (see [5, Thm. 1.2]):

Proposition 3.6. Let $G$ be reductive, $H \leq G$ a closed subgroup, and $C, D$ conjugacy classes of $G$.

(a) If $(C \cap H)(D \cap H)$ hits infinitely many semisimple $H$-classes, then $C D$ hits infinitely many semisimple $G$-classes.

(b) If $H$ is reductive and $(C \cap H)(D \cap H)$ hits infinitely many $H$-classes, then $C D$ hits infinitely many $G$-classes.

Theorem 3.7. Let $G$ be an almost simple algebraic group of adjoint type over an algebraically closed field $k$ of characteristic $p \geq 0$ with connected component $G^{\circ}$. Let $C_{1}, C_{2}$ be non-trivial unipotent $G^{\circ}$-classes of $G=\left\langle C_{1}, C_{2}\right\rangle$ such that $x y$ is unipotent for all $x \in C_{1}$, $y \in C_{2}$. Then $p \in\{2,3\}$ and (up to order of $C_{1}, C_{2}$ ) either:

(1) $G=G^{\circ}$ is connected, $C_{1}$ contains long root elements and one of:

(a) $p=2, G=\operatorname{Sp}_{2 n}(k)=\operatorname{Sp}(V)$ with $n \geq 2$, and $C_{2}$ contains involutions $y$ with $(y v, v)=0$ for all $v$ in $V$; or

(b) $(G, p)=\left(F_{4}, 2\right)$ or $\left(G_{2}, 3\right)$, and $C_{2}$ contains short root element; or

(2) $G$ is the extension of $G^{\circ}$ by a graph automorphism of the Dynkin diagram of order $p$, $\mathrm{C}_{2}$ is the class of this quasi-central automorphism, and one of:

(c) $p=2, G=A_{2 n-1}(k) .2$ with $n \geq 2, C_{1}$ consists of long root elements and $C_{2}$ is the class of the graph automorphism with centralizer $C_{n}(k)$;

(d) $p=2, G=D_{n}(k) .2, C_{1}$ contains involutions as given in (1)(a) for $C_{n}(k)$ and $C_{2}$ consists of transvections (the class of graph automorphisms with centralizer $\left.B_{n-1}(k)\right)$;

(e) $p=2, G=E_{6}(k) .2, C_{1}$ is the class of long root elements and $C_{2}$ is the class of the graph automorphism with centralizer $F_{4}(k)$; or

(f) $p=3, G=D_{4}(k) .3, C_{1}$ is the class of long root elements and $C_{2}$ is the class of the graph automorphism with centralizer $G_{2}(k)$.

Moreover, in all these cases, $\langle x, y\rangle$ is unipotent for all $x \in C_{1}, y \in C_{2}$.

Proof. By [7, Thm. 1.1] we may assume that $G$ is disconnected and $C_{2}$, say, lies in $G \backslash G^{\circ}$. So either $p=2$ and $G^{\circ}$ has type $A_{n}(n \geq 2), D_{n}(n \geq 4)$, or $E_{6}$, or $p=3$ and $G^{\circ}$ has type $D_{4}$. By Examples 3.2 3.5 above, all the cases described in (2)(c)-(2)(f) do give examples.

Suppose that $C_{1}$ also consists of outer elements. By Corollary 2.4 there is a unique outer unipotent class lying in the closure of all other unipotent classes in a fixed coset. By taking closures we may assume that $C_{1}, C_{2}$ are these. If $p=3$ and $C_{1}=C_{2}$ then a computation in $\mathrm{SO}_{8}^{+}(3) .3$ shows that the product contains non-unipotent classes. Else, $C_{2}$ contains the inverses of $C_{1}$, and since outer quasi-semisimple elements normalize, but do not centralize, a maximal torus of $G^{\circ}$, we obtain non-trivial semisimple products.

So we may assume that $C_{1} \subset G^{\circ}$. Suppose for the moment that $p=2$.

First consider the case that $G^{\circ}=A_{n}(k), n \geq 2$. Suppose that $C_{1}$ does not consist of transvections. Then by taking closures, we may assume that $C_{1}$ either consists of elements with one Jordan block of size 3 or two Jordan blocks of size 2 , and $C_{2}$ consists of quasi-semisimple elements. Thus, it suffices to work in $A_{2}(k)$ or $A_{3}(k)$. Note that all inner unipotent classes and all classes of involutory graph automorphisms intersect 
$A_{2}(2) .2 \cong \mathrm{PGL}_{2}(7)$ resp. $A_{3}(2) .2 \cong \mathfrak{S}_{8}$ and we obtain a contradiction. So $C_{1}$ consists of transvections. If $n$ is even, we may assume that $C_{2}$ contains quasi-semisimple elements and reduce to the case of $A_{2}(k) .2$. Computing in $A_{2}(2) .2$, we see that there are no examples. If $n \geq 3$ is odd, by [15] all outer unipotent classes except for the quasi-semisimple one, contain the transpose inverse graph automorphism in their closure. So we may reduce to the case of $A_{3}(2) .2$ to see that $C_{2}$ must correspond to transpositions in the symmetric group and so must be the class of the graph automorphisms with centralizer $C_{n}(k)$, the quasi-semisimple class. We thus arrive at case (2)(c).

Next consider the case that $G^{\circ}=D_{n}(k)$. Suppose that $C_{2}$ does not consist of transvections in the natural $2 n$-dimensional representation of $D_{n}(k) .2$. By passing to closures, we may assume that $C_{2}$ contains elements with exactly three nontrivial Jordan blocks of size 2 in the natural $2 n$-dimensional representation. In particular, we see that elements of $C_{2}$ leave invariant a nondegenerate 6-dimensional space. Passing to the closure, we may assume that $C_{1}$ consists of long root elements. In particular, we can reduce to the case of $D_{3}(k)=A_{3}(k)$ for which we already saw that there are no such examples.

So we may assume that $C_{2}$ consists of transvections. We claim that $C_{1}$ contains involutions $x$ so that $(x v, v)=0$ for all $v \in V$, the natural $2 n$-dimensional module for $C_{n}(k) \geq D_{n}(k) .2$ as in $(2)(\mathrm{d})$. If $C_{1}$ consists of involutions which do not have that property, then any $x \in C_{1}$ leaves invariant a nondegenerate 2-dimensional space on which it acts as a transvection. Thus, $x y\left(y \in C_{2}\right)$ can have odd order on that 2-dimensional space.

If $C_{1}$ does not consist of involutions, then going to the closure allows us to assume that $C_{1}$ contains elements of order 4 . It follows that any $x \in C_{1}$ leaves invariant a nondegenerate subspace of dimension 6 or 8 (acting as an element of order 4 ). Thus, we can reduce to either $D_{3}(k)=A_{3}(k)$ or $D_{4}(k)$. In the first case, we are done by the result for type $A$. In the second case, we note that every class of elements of order 4 in $\mathrm{SO}_{8}(k)$ intersects $\mathrm{SO}_{8}^{+}(2)$ and so we may compute structure constants in $\mathrm{GO}_{8}^{+}(2)$ to conclude that there are no such examples.

Now suppose that $G^{\circ}=E_{6}(k)$. First suppose that elements of $C_{2}$ do not have centralizer $F_{4}(k)$. By taking closures, we may assume that $C_{1}$ consists of long root elements in $E_{6}(k)$. By [15, p. 250] all outer unipotent classes except for the quasi-semisimple one contain the graph automorphism with centralizer $C_{4}(k)$ in their closure. Let $\tau$ be an involution with centralizer $F_{4}(k)$. Let $x \in F_{4}(k)$ be a long root element in $E_{6}(k)$ such that $\tau x \in C_{2}$. Then by the result for the connected group $F_{4}(k)$ there is $y \in C_{1} \cap F_{4}(k)$ with $x y$ of odd order.

So we may assume that elements of $C_{2}$ have centralizer $F_{4}(k)$. If $C_{1}$ consists of long root elements then we are in case $(2)(\mathrm{e})$. Else, by taking closures, we may assume that $C_{1}$ is the class $2 A_{1}$ [15, p. 247]. This class has representatives in the Levi subgroup $A_{5}(k)$ and so the result follows by that case.

Finally, consider the case $p=3$ with $G^{\circ}=D_{4}(k)$. Suppose that elements of $C_{2}$ do not have centralizer $G_{2}(k)$. By taking closures, we may assume that $C_{1}$ consists of long root elements. Let $\tau$ be the graph automorphism with centralizer $G_{2}(k)$. Choose $x, y \in G_{2}(k)$ which are long root elements of $D_{4}(k)$ such that $x y$ is not a 3-element (by the result for connected groups) and $\tau x \in C_{2}$. Then $\tau x y$ is not a 3-element either. So we may assume that elements of $C_{2}$ have centralizer $G_{2}(k)$. Note that the graph automorphism fuses the three non-trivial unipotent classes of $G^{\circ}$ of second smallest dimension (see [15, p. 239]), so if $C_{1}$ is not as in case (2)(f), then by closure we may assume that $C_{1}$ is one of these 
classes. But computation of structure constants in the finite subgroup $\mathrm{SO}_{8}^{+}(3) .3$ shows that this does not give an example.

Note that in all the exceptions in the statement, the group generated by $x \in C_{1}, y \in C_{2}$ is always unipotent, by Examples [3.2 [3.5, resp. by the examples in [7]. This completes the proof of Theorem 3.7 .

\section{Products of Conjugacy Classes in Disconnected Algebraic Groups}

In [7, Thm. 1.1] we classified pairs of conjugacy classes $C, D$ in a simple algebraic group such that the product $C D$ consists of a finite union of conjugacy classes (equivalently, the semisimple parts of elements in $C D$ form a single conjugacy class). We build on the results from the previous section to extend this to the disconnected case.

First we give some examples.

Example 4.1. Let $S=\mathrm{SL}_{2 n}(k), n \geq 2$, with $k$ algebraically closed. Let $x \in S$ be any element that is (up to scalar) a pseudoreflection, $y$ a graph automorphism with centralizer $\operatorname{Sp}_{2 n}(k)$ and $G=\langle S, y\rangle$. Then by [7, Ex. 7.2], there is a single $G$-orbit on $x^{S} \times y^{S}$, whence $x^{S} y^{S}$ is a single conjugacy class. In particular, if $k$ is not of characteristic 2 , and $x$ is a 2-element, $\langle x, y\rangle$ is a 2-group.

Example 4.2. Let $G=\mathrm{GO}_{2 n}(k), n \geq 4$, with $k$ algebraically closed. Suppose that $x \in G$ is an element whose centralizer is $\mathrm{GL}_{n}(k)$ and $y \in G$ is a reflection. As $\mathrm{GL}_{n}(k)$ acts transitively on non-degenerate 1-spaces of the natural module for $G$, we have $G=$ $\mathrm{GO}_{2 n-1}(k) \mathrm{GL}_{n}(k)$ and so $G$ has a single orbit on $x^{G} \times y^{G}$. If $k$ is not of characteristic 2 and $x$ has finite order $m$, then $x y^{g}$ will have order $2 m /(2, m)$.

Example 4.3. Let $G=\mathrm{GO}_{2 n}(k), n \geq 4$, with $k$ algebraically closed of characteristic not 2. Suppose that $x$ is unipotent with all Jordan blocks of size at most 2 and $y$ is a reflection in $G$. Then $x$ and $y$ are both trivial on a totally singular subspace of dimension $n-1$ of the natural module. Let $P$ denote the parabolic subgroup stabilizing this space. Then $x$ lies in the unipotent radical of $P$, whence $x y$ has constant semisimple part $y$. Thus we get an example with $C_{1}=x^{G}, C_{2}=y^{G}$.

Example 4.4. Let $G=\mathrm{SO}_{8}(k) .3$ with $k$ algebraically closed of characteristic not 3 . Let $C_{1}$ be the class of root elements of $G^{\circ}$, and $y \in C_{2}$, the class of graph automorphisms with centralizer $H=G_{2}(k)$ in $G^{\circ}$. Let $s \in H$ be an element of order 3 with $C_{H}(s)=\operatorname{SL}_{3}(k)$. By looking at the centralizer in the adjoint action on the Lie algebra of $G^{\circ}$ one sees that $s y$ is $G$-conjugate to $y$. Note that $C_{1} \cap H$ are long root elements in $H$. Thus by [7, Ex. 6.2] the product $\left(C_{1} \cap H\right) s$ contains an element $u s$ with $u$ regular unipotent in $C_{H}(s)=\mathrm{SL}_{3}(k)$, with centralizer dimension 4 in $H$. Thus $x s y \sim u s y$ for some $x \in C_{1}$, with $[u, s y]=1$ and of coprime order. It follows that $\operatorname{dim} C_{G}(x s y)=\operatorname{dim} C_{G}(u s y)=\operatorname{dim} C_{H}(u)=4$, so that $C_{1} C_{2}$ contains a class of $\operatorname{dimension} \operatorname{dim} G-4=24=\operatorname{dim} C_{1}+\operatorname{dim} C_{2}$. An application of Lemma 3.1 shows that $C_{1} C_{2}$ is a union of finitely many classes.

Similarly, let $G=E_{6}(k) .2$ with $k$ algebraically closed of characteristic not 2 . Let $C_{1}$ be the class of root elements of $G^{\circ}$ and $y \in C_{2}$, the class of graph automorphisms with centralizer $H=F_{4}(k)$ in $G$. Let $s \in H$ be an involution with $C_{H}(s)=B_{4}(k)$. By looking at the adjoint action one sees that $s y$ is $G$-conjugate to $y$. Again, $C_{1} \cap H$ consists of long root elements in $H$. Thus by [7, Ex. 6.4] the product $\left(C_{1} \cap H\right) s$ contains elements $u \in B_{4}(k)$ 
whose square is a short root element, with centralizer dimension $30 \mathrm{in} H$. Arguing as before, we see that $C_{1} C_{2}$ contains a class of dimension $\operatorname{dim} G-30=48=\operatorname{dim} C_{1}+\operatorname{dim} C_{2}$, and we conclude by Lemma 3.1 that $C_{1} C_{2}$ is a finite union of classes.

Example 4.5. Let $G=\mathrm{SO}_{8}(k) \cdot \mathfrak{S}_{3}$ with $k$ algebraically closed. Let $C_{1}, C_{2}$ be $G^{\circ}$-classes of graph automorphisms with centralizer $H=B_{3}(k)$ in $G^{\circ}$ lying in two different $G^{\circ}$-cosets. Then $C_{1}, C_{2}$ both have dimension 7 , while any class in the coset of order 3 has dimension at least 14 , which is attained for the quasi-central class of graph automorphisms of order 3 . So the product consists of that single class by Lemma 3.1. See Section 7 for more on this.

Theorem 4.6. Let $G$ be an almost simple algebraic group of adjoint type over an algebraically closed field $k$ of characteristic $p \geq 0$ with connected component $G^{\circ}$. Let $C_{1}$ and $C_{2}$ be nontrivial $G^{\circ}$-conjugacy classes in $G=\left\langle C_{1}, C_{2}\right\rangle$. Suppose that $C_{1} C_{2}$ consists of finitely many conjugacy classes. Then one of the following holds:

(1) $C_{1}, C_{2}$ are unipotent classes and we are in one of the cases of Theorem 3.7; or

(2) $G=G^{\circ}$ is connected, $C_{2}$ is unipotent, and one of

(a) $G=\operatorname{Sp}_{2 n}(k), n \geq 2, p \neq 2, C_{1}$ contains involutions and $C_{2}$ contains long root elements;

(b) $G=\mathrm{SO}_{2 n+1}(k), n \geq 2, p \neq 2, C_{1}$ contains reflections (modulo scalars) and $C_{2}$ contains unipotent elements with all Jordan blocks of size at most 2;

(c) $G=F_{4}, p \neq 2, C_{1}$ contains involutions with centralizer $B_{4}(k)$ and $C_{2}$ contains long root elements;

(d) $G=G_{2}, p \neq 3, C_{1}$ contains elements of order 3 with centralizer $\mathrm{SL}_{3}(k)$ and $C_{2}$ contains long root elements; or

(3) $G$ is disconnected, $C_{2}$ contains quasi-semisimple graph automorphisms, and one of

(e) $\left[G: G^{\circ}\right]=2, G^{\circ}=A_{2 m-1}(k), m>1, C_{1}$ contains (semisimple) pseudoreflections and $C_{2}$ contains graph automorphisms with centralizer $C_{m}(k)$;

(f) $\left[G: G^{\circ}\right]=2, G^{\circ}=A_{2 m-1}(k)$ with $p \neq 2, m>1, C_{1}$ contains transvections and $C_{2}$ contains graph automorphisms with centralizer $C_{m}(k)$;

(g) $\left[G: G^{\circ}\right]=2, G^{\circ}=D_{n}(k), n \geq 4, C_{1}$ contains semisimple elements with centralizer $A_{n-1}(k)$ and $C_{2}$ contains graph automorphisms with centralizer $B_{n-1}(k)$;

(h) $\left[G: G^{\circ}\right]=2, G^{\circ}=D_{n}(k)$ with $p \neq 2, n \geq 4, C_{1}$ contains unipotent elements with all Jordan blocks of size at most 2 and $C_{2}$ contains graph automorphisms with centralizer $B_{n-1}(k)$;

(i) $\left[G: G^{\circ}\right]=2, G^{\circ}=E_{6}(k)$ with $p \neq 2, C_{1}$ contains long root elements and $C_{2}$ contains graph automorphisms with centralizer $F_{4}(k)$;

(j) $\left[G: G^{\circ}\right]=3, G^{\circ}=D_{4}(k)$ with $p \neq 3, C_{1}$ contains long root elements and $C_{2}$ contains graph automorphisms with centralizer $G_{2}(k)$; or

(k) $\left[G: G^{\circ}\right]=6, G^{\circ}=D_{4}(k), C_{1}$ and $C_{2}$ are classes in different cosets modulo $G^{\circ}$ of graph automorphisms of order 2.

Note that cases (f), (h), (i) and (j) are direct analogues of the unipotent pairs in Theorem 3.7. We analyze the case 3(k) completely in Section 7 , see Proposition 17.4, If we consider $G$-classes instead of $G^{\circ}$-classes, we get the same examples except for the ones in $3(\mathrm{k})$.

The proof of Theorem 4.6 will be given in a series of five lemmas. First note that if $G$ is connected, the result was shown in [7, Thm. 1.1]. So we may and will assume that $G^{\circ}$ 
is one of $A_{n}(k)(n \geq 2), D_{n}(k)(n \geq 4)$, or $E_{6}(k)$. Let $r=\left[G: G^{\circ}\right]$. We may assume that $C_{2}$ consists of outer automorphisms. We consider various cases.

Lemma 4.7. The claim in Theorem 4.6 holds when neither of the classes $C_{1}, C_{2}$ is contained in $G^{\circ}$.

Proof. Passing to closures, we may first assume by Theorem 2.3 that $C_{1}, C_{2}$ are both quasisemisimple. Then there exist $(x, y) \in C_{1} \times C_{2}$ normalizing a common Borel subgroup $B$ and a maximal torus $T \leq B$. Let $S:=[y, T]$. Then $y S \subseteq C_{2}$ and $S$ is infinite as $y \notin C_{G}(T)=C_{G^{\circ}}(T)$. Let $m$ denote the order of $z=x y$ in $G / G^{\circ}$. If $m=1$ then $x y S \subset T \cap C_{1} C_{2}$ is infinite, so contains infinitely many classes.

If $m>1$ then necessarily $G^{\circ}=D_{4}(k)$. If $m=2$ then $(x y t)^{2}=z^{2} t^{z} t$, for $t \in S$, is finite only if $z$ acts as -1 on $S$. Note that this configuration can only occur when $C_{1}, C_{2}$ lie in cosets of $G^{\circ}$ of order 2, 3 respectively. Explicit computation shows that here $z$ does not act as -1 on $S$.

Thus we have that $m=3$. If $C_{1}, C_{2}$ lie in the same coset of order 3 , then $(x y t)^{3}=$ $\left\{z^{3} t^{z^{2}} t^{z} t \mid t \in S\right\}$. Again, explicit computation shows that this is not finite for suitable $N_{G}(T)$-conjugates of $x, y$. The only remaining possibility is that $G / G^{\circ} \cong \mathfrak{S}_{3}, x, y$ lie in two different cosets of order 2 , as in $(3)(\mathrm{k})$ of the conclusion.

Lemma 4.8. Let $G=A_{1}(k) \backslash Z_{r}$, the wreath product of $A_{1}(k)$ with the cyclic group of prime order $r$. Let $C_{1} \subset G^{\circ}$ be a conjugacy class whose projection to at least two factors is non-central, and $C_{2} \subset G \backslash G^{\circ}$ any conjugacy class. Then:

(a) $C_{1} C_{2}$ consists of infinitely many conjugacy classes.

(b) $\left[C_{1}, C_{2}\right]$ consists of infinitely many conjugacy classes.

Proof. Let $\sigma$ denote a generator of the cyclic subgroup of $G$ permuting the $A_{1}(k)$-factors. Any outer element is conjugate to one of the form $\sigma(1, \ldots, 1, x)$ for some $x \in A_{1}(k)$. An easy computation with such elements shows (a) and (b), using that the product of any two non-central classes of $A_{1}(k)$ meets infinitely many classes.

Lemma 4.9. The claim in Theorem 4.6 holds when $G^{\circ}=A_{n}(k)$.

Proof. By our initial reductions and Lemma 4.7 we may assume that $r=2, C_{1} \subset G^{\circ}$ and $C_{2}$ is outer.

Case 1. $n$ is even.

In this case, we claim there are no examples. By taking closures, we may assume that $C_{2}$ contains quasi-semisimple elements. Thus $x_{2} \in C_{2}$ normalizes a maximal torus, hence any Levi subgroup $L$ of $G^{\circ}$ invariant under the graph automorphism. Again by closure, $C_{1}$ consists of semisimple elements or of transvections. In either case, we see that one can find $x_{i} \in C_{i}$ normalizing a subgroup $A_{2}(k)$ and acting nontrivially. Thus, it suffices to take $n=2$. Now any quasi-semisimple element of $A_{2}(k)$ normalizes a maximal torus, so is of the form $x t, t \in T$, for some fixed quasi-semisimple element $x$. It is then a straightforward matrix computation to see that $C_{1} C_{2}$ always meets elements with distinct characteristic polynomial, so with distinct semisimple part.

Case 2. $n=2 m-1 \geq 3$ is odd.

Arguing as above we can reduce to $x_{1}, x_{2}$ lying in a Levi subgroup of type $A_{3}$. Inspection shows that then $x_{2}$ has to be an involution. We claim that $C_{2}$ consists of involutions with 
centralizer $C_{m}(k)$. Suppose not. Then by [3], Tab. 4.5.1], elements in $C_{2}$ have centralizer $D_{m}(k)$. We may assume that $x_{1} \in C_{1}$ is either semisimple or a transvection, and then after conjugation we have $x_{1}, x_{2}$ in a disconnected subgroup with connected component a Levi subgroup of type $A_{n-1}$, where no example exists by the first case.

If $x_{1}$ is semisimple, there is some root of $G$ which is non-trivial on $x_{1}$. Since $G^{\circ}$ has just one root length, after conjugation we may assume that $x_{1}$ lies in an $x_{2}$-stable Levi subgroup $L$ of type $D_{2}$ but not in its center, with $x_{2}$ swapping the two factors. Moreover, if no eigenspace of $x_{1} \in C_{1}$ has dimension $n-1$, then we may arrange that the image of $x_{1}$ in both factors is non-trivial. In that case, by Lemma 4.8 the product $C_{1} C_{2}$ meets infinitely many semisimple classes inside the wreath product $A_{1}(k)<2$. On the other hand, if $x_{1}$ is a pseudo-reflection (modulo scalars), we get case (3)(e) of the assertion by Example 4.1.

If $x_{1}$ is unipotent, we may assume that $p>2$ since otherwise we are in the situation of Theorem 3.7. If $x_{1}$ is a transvection, then we get case (f) by Example 3.2. Else, by closure we may assume that $x_{1}$ has two Jordan blocks of size 2 or one of size 3 . In the first case, we may reduce to $A_{3}$, in the second to $A_{2}$, and no examples arise.

If $x_{1}$ is neither unipotent nor semisimple, then by the previous arguments it must be the commuting product of a pseudo-reflection with a transvection. In this case, we may again reduce to the wreath product $A_{1}(k)<2$ and apply Lemma 4.8 .

Lemma 4.10. The claim in Theorem 4.6 holds when $G^{\circ}=D_{n}(k), n \geq 4$.

Proof. As in Lemma 4.9 we may assume that $C_{1} \subset G^{\circ}$ and $C_{2}$ is outer, so $r \in\{2,3\}$.

Case 1. $r=2$.

First suppose that $x_{1}$ is semisimple but not as in the conclusion (g). By taking closures we may assume $x_{2}$ is quasi-semisimple. Then we can reduce to $\mathrm{GO}_{4}(k)=A_{1}(k) \prec 2$. Moreover $x_{1}$ projects to elements which are nontrivial in each factor $A_{1}(k)$ whence $C_{1} C_{2}$ contains infinitely many classes by Lemma 4.8. Next suppose that $x_{1}$ has centralizer $\mathrm{GL}_{n}(k)$. If $x_{2}$ is quasi-semisimple but not a reflection, we can reduce to $D_{3}(k)=A_{3}(k)$ and invoke Lemma 4.9. So we may assume that the semisimple part of $x_{2}$ is a reflection. If its unipotent part is nontrivial, then by taking closures we may assume it is a root element in $\mathrm{SO}_{2 n-1}(k)$ and so either has two Jordan blocks of size 2 or one Jordan block of size 3. Again, we can reduce to $D_{3}(k)$ where this cannot happen. When $x_{2}$ is a reflection, we arrive at $(3)(\mathrm{g})$ by Example 4.2 ,

If $x_{1}$ is unipotent with all Jordan blocks of size at most 2 and $x_{2}$ is a reflection, then this gives $(3)(\mathrm{h})$ by Example 4.3. If $x_{2}$ does not consist of reflections, we may again reduce to $D_{3}(k)$ to obtain a contradiction. If $x_{1}$ has a Jordan block of size at least 3 , by taking closures we may assume that it has just one Jordan block and $x_{2}$ is semisimple, and then we may reduce to a subgroup $D_{3}(k)$ for which there are no such examples.

Finally, suppose that $x_{1}$ is a mixed element. The above argument shows that $x_{2}$ is a reflection, the semisimple part of $x_{1}$ has centralizer $\mathrm{GL}_{n}(k)$ and its unipotent part has Jordan blocks of size at most 2. Again, we can reduce to $D_{3}(k)$ to rule out this case.

Case 2. $r=3$, so $n=4$.

First assume that $x_{2} \in C_{2}$ is quasi-semisimple. If $x_{1}$ is unipotent, then $x_{1}, x_{2}$ lie in the normalizer of a maximal parabolic subgroup $P$ of type $A_{1}^{3}$. If $x_{1}$ is not a long root element, 
the image of $x_{1}$ in the Levi subgroup $L$ of $P$ is non-trivial in at least two of the factors, whence the product $C_{1} C_{2}$ meets infinitely many classes in $L$ by Lemma 4.8. If $x_{1}$ is a long root element, we may conjugate $x_{1}, x_{2}$ into the graph centralizer $G_{2}(k)$. By [7, Thm. 5.11] the only example there is for $x_{2}$ to be the 3-element with centralizer $\mathrm{SL}_{3}(k)$. But by the argument in Example 4.4, all such elements fuse into the class of graph automorphisms with centralizer $G_{2}(k)$, so we arrive at $(3)(\mathrm{j})$.

Any non-central semisimple element has a conjugate which is non-trivial in at least two factors of the Levi subgroup $L$ of type $A_{1}^{3}$ and thus gives infinitely many classes there. Finally, if $x_{1}$ is a mixed element, we may assume that its unipotent part is a long root element, and then again its image in $L$ is non-trivial in at least two factors.

Finally, if $x_{2}$ is not quasi-semisimple, by the previous argument and taking closures, we may assume that $x_{2}=x u=u x$ where $u$ is a long root element and $x$ is quasi-semisimple of order 3 , and $x_{1}$ is a long root element. In particular if we take $x_{1}$ centralizing $x$, we see that $x_{1}$ and $u$ are conjugate in $G_{2}(k)$ and since $u^{G_{2}(k)} u^{G_{2}(k)}$ hits infinitely many classes, we are done.

Lemma 4.11. The claim in Theorem 4.6 holds when $G^{\circ}=E_{6}(k)$.

Proof. Here we have $r=2$. By closure we may assume that $x_{1} \in C_{1}$ is semisimple or unipotent of type $A_{2}$, or with unipotent part a long root element, and $x_{2} \in C_{2}$ is quasisemisimple. Hence we may arrange so that $x_{1}, x_{2}$ are contained in a Levi subgroup of type $A_{5}$, which is normalized by the graph automorphism. By Lemma 4.9 this forces $x_{2}$ to be an involution. There are two classes of outer involutions in $G$, with centralizers $F_{4}(k)$ resp. $C_{4}(k)$. The outer involutions of $A_{5}(k) .2$ with centralizer of type $D_{3}$ fuses into the outer class with centralizer of type $C_{4}$ (since $A_{5}(k)$ has composition factors of dimensions $6,6,15$ on the 27-dimensional module for $E_{6}(k)$, and so has $D_{3}(k)$, while $F_{4}(k)$ has a 1-dimensional constituent), so by our considerations in Lemma 4.9, $x_{2}$ must have centralizer $F_{4}(k)$, and $x_{1}$ must be a long root element. This occurs by Example 4.4.

We have now discussed all possibilities for $G$ and thus completed the proof of Theorem 4.6 .

We note the following relation between the examples in disconnected groups in Theorem 4.6 and the ones in connected groups in [7, Thm. 1.1]:

Proposition 4.12. Let $G$ be a simple algebraic group, $\tau$ a graph automorphism of $G$ with reductive centralizer $H=C_{G}(\tau)$. Suppose moreover that for some $1 \neq g \in H, \tau$ and $g \tau$ are $G$-conjugate. If $C:=\tau^{G}$ and $D \subset G$ are classes such that $C D$ consists of only finitely many classes in $G \tau$, then the same holds for $C_{1}=g^{H}, D_{1}=D \cap H$ in $H$.

Proof. Clearly, $C_{1} D_{1} \tau=g^{H} \tau D_{1}=(g \tau)^{H} D_{1} \subset C D$, and if the latter meets only finitely many $G$-classes, then the former only meets finitely many $H$-classes, see Proposition 3.6.

This applies to cases (e), (f), (h), (i) and (j) in Theorem 4.6.

\section{Commutators}

In this section we turn to commutators of pairs of conjugacy classes. We first investigate the commutator of a single class and thus prove Theorem 1.4. The proof follows the ideas in $[7, \S 5]$. 
It is convenient to recall the following result [4] (which answered a question of Katz before it was asked - see [8, 2.16.7]).

Theorem 5.1. Let $G$ be a simple algebraic group of type $A$ or $C$. Suppose that $x, y \in G$ with $[x, y]$ a long root element (i.e., a transvection). Then $\langle x, y\rangle$ is contained in a Borel subgroup.

Proof. We may as well work in $\mathrm{SL}_{n}$. Suppose that $z:=x^{-1} y^{-1} x y$ is a long root element. Write $z=I+N$ where $N$ is a nilpotent rank one matrix. Then $x y-y x=y x N$ is a rank one matrix. Now apply the main result of [4].

Proof of Theorem 1.4. Let $G$ be an almost simple algebraic group over $k$ and $C$ a $G^{\circ}$ conjugacy class of $G$ outside $Z\left(G^{\circ}\right)$. Let $w: C \times C \rightarrow G$ be the map defined by $w(x, y)=$ $[x, y]$.

Since $C$ is an irreducible variety, if the result fails, the semisimple part of $[x, y]$ for $(x, y) \in C \times C$ would be constant. Of course, $1=[x, x]$ and therefore the image of $w$ must be contained in the set of unipotent elements of $G$. If $[C, C]$ consists of finitely many classes, the same is true for its closure, so we may also replace $C$ by any class in its closure and so assume that $C$ is semisimple or unipotent.

First suppose that $G \cong A_{1}(k)$. We claim that it suffices to show the result for $\mathrm{SL}_{2}(k)$. Indeed, lift $C$ to a class of $\mathrm{SL}_{2}(k)$; the image of $w$ is irreducible and contains 1 , so still all commutators are unipotent (not just unipotent modulo the center).

Choose $(x, y) \in C \times C$ so that $x$ and $y$ are not contained in a common Borel subgroup (since any non-central element lies in at most two Borel subgroups, this clearly can be done). By Theorem [5.1, $[x, y]$ is not unipotent.

Next suppose that $G=G^{\circ}$ is connected. If $C$ contains semisimple elements, we can choose $x \in C \cap T$ where $T$ is a maximal torus and so $x$ does not centralize some root subgroup $U$. Let $U^{-}$be the corresponding negative root subgroup. Then $\left\langle T, U, U^{-}\right\rangle$is a product of a torus with an $A_{1}(k)$, whence the result follows.

If $C$ is unipotent, by closure we may assume that it consists of root elements. Then we just work in $\left\langle U, U^{-}\right\rangle$where $U$ is a root subgroup generated by elements of $C$ and again the result follows by the $A_{1}(k)$ result.

Finally suppose that $G$ is disconnected. We may assume that $C$ consists of outer automorphisms, and by closure that it is closed. In particular, elements of $C$ are quasisemisimple by Theorem 2.3. By definition, any such element normalizes, but does not centralize, a maximal torus of $G^{\circ}$, and thus there exist non-trivial semisimple commutators of elements in $C$.

It follows easily from the previous result that if $C$ is a noncentral conjugacy class of $\ell$-elements for some prime $\ell$, then commutators of pairs of elements of $C$ cannot consist only of $\ell$-elements (this is related to the question of Shumyatsky).

If we take distinct classes, there are examples in [7] showing that the commutators could always be unipotent. Arguing as in [7], we can actually classify all such pairs.

Theorem 5.2. Let $G$ be an almost simple algebraic group over an algebraically closed field $k$ of characteristic $p \geq 0$. Let $C, D$ be non-central $G^{\circ}$-conjugacy classes in $G$ with $G=\langle C, D\rangle$. Assume that $C, D$ do not lie in distinct cosets of order 2 if $G=D_{4}(k) \cdot \mathfrak{S}_{3}$. 
Then

$$
[C, D]:=\{[x, y] \mid x \in C, y \in D\}
$$

is the union of finitely many conjugacy classes if and only if $C, D$ are as given in Theorem 3.7 or as in Theorem 4.6(a)-(j). In particular, $C \neq D$ in this case.

Proof. Let $w: C \times D \rightarrow G$ be as in the previous proof. By irreducibility the semisimple part of elements in $[C, D]$ has to be constant.

We can now essentially follow the proof in [7], respectively the proofs of Theorems 3.7 and 4.6. First assume that $G$ is connected. For $G=\mathrm{SL}_{2}(k)$, choosing $(x, y) \in C \times D$ in the same Borel subgroup, $[x, y]$ is clearly unipotent. On the other hand, choose $x$ and $y$ so that they are not contained in a common Borel subgroup. Then by Theorem [5.1, $[x, y]$ is not unipotent. So there's no example for $\mathrm{SL}_{2}(k)$.

If $[C, D]$ consists of finitely many classes, the same is true for its closure, so we may also replace $C$ and $D$ by any classes in their closures and so assume that each is semisimple or unipotent. Moreover, if one of them is a unipotent class, we may assume that it consists of root elements. We can now argue exactly as in the proof of [7, Thm. 5.11] to rule out every configuration apart from those in [7, Thm. 5.11(2)-(6)], except for the case that $p \neq 2, G=\operatorname{Sp}_{4}(k)$, and the semisimple part of $c$ is an involution. But again by working inside the subsystem subgroup $A_{1}(k)^{2}$ we see that $[C, D]$ can have infinitely many distinct semisimple parts by Lemma 4.8.

In all the exceptions of [7, Thm. 5.11(2)-(6)], any pair $(x, y) \in C \times D$ lies in some common Borel subgroup of $G$ by [7, Cor. 5.14], whence the commutator $[x, y]$ is unipotent and so $[C, D]$ consists of finitely many (unipotent) classes.

Now assume that $G$ is not connected, but $C, D$ are unipotent. Then the same would have to hold for all classes in $[C, D]$, since all unipotent elements have conjugates in a maximal unipotent subgroup of the normalizer of a Borel subgroup. Following the arguments in the proof of Theorem 3.7] we see that the assertion follows once we show that the only pairs of 2 -power classes in $\mathfrak{S}_{8}, \mathrm{PGL}_{2}(7), \mathrm{GO}_{8}^{+}(2)$ with commutator consisting of 2-elements are those in the conclusion. This can be checked by direct computation.

Finally assume that $G$ is disconnected and not both $C, D$ are unipotent. Here, we go through the proof of Theorem 4.6. If both classes are outer (and quasi-semisimple), then we may find representatives $x, y$ normalizing a maximal torus $T$, as in the proof of Lemma 4.7. First assume that $x, y$ lie in the same cyclic subgroup of $G / G^{\circ}$. Then with $S=\left[T, y^{-1}\right]$ and $R=[x, S]$ we have $[x, S y]=\left\{x^{-1} y^{-1} x r y \mid r \in R\right\} \subseteq\left[C_{1}, C_{2}\right] \cap T$, which is always infinite, since $x$ may be chosen not to centralize the commutator space of $y^{-1}$. The only remaining case is when $G=D_{4} \cdot \mathfrak{S}_{3}$ with $x, y$ in non-trivial cosets of different order. Here, the cube of the commutator $[x, S y]$ hits infinitely many classes in $T$ since $\left\{r \in R \mid r^{z^{2}} r^{z} r\right\}$ is not finite, for $z=\left[y^{-1}, x\right]$.

Thus, $C$ is inner, say. As in the proof of Lemma 4.9 we are done for $A_{n}(k)$ once we have shown the claim for $n=2$ and $n=3$, where it is a direct matrix calculation. The arguments for all other types go through unchanged.

In the case of commutators between $G^{\circ}$-classes in two different cosets of order 2 in $D_{4}(k) \cdot \mathfrak{S}_{3}$, there are many further examples, see Example 7.6 below. 


\section{Double cosets, Products and Commutators}

The aim of this section is the Characterization Theorem 1.1 for various finiteness properties. For this we need to investigate some of the examples from the previous three sections a bit more closely.

Proposition 6.1. Let $G=\mathrm{GO}_{2 n}(k), n \geq 3$, with $k$ algebraically closed of characteristic $p$. Let $x \in G^{\circ}$ be unipotent such that either

(1) $p \neq 2$ and all Jordan blocks of $x$ have size at most 2 ; or

(2) $p=2, x^{2}=1$ and $(x v, v)=0$ for all $v$ in the natural module $V$ for $G$ (under the associated alternating form).

Then $C_{G}(x)$ has at most two orbits on nondegenerate 1-spaces in $V$.

Proof. We claim that any nondegenerate 1-space is conjugate under $C_{G}(x)$ to an element in a nondegenerate 4 -space or 6 -space that is $x$-invariant.

Write $V=V_{1} \perp V_{2}$ where $V_{1}$ and $V_{2}$ are $x$-invariant and $x$ is trivial on $V_{1}$ and has all Jordan blocks of size exactly 2 on $V_{2}$.

It suffices to deal with each space separately. In the first case the centralizer contains $\mathrm{GO}\left(V_{1}\right)$ and we can move any 1 -space into any nondegenerate 2 -space. So we need to consider $V_{2}$. We claim that any vector is $C_{G}(x)$-conjugate to an element of some $x$ invariant nondegenerate 4 -space.

In both cases the fixed space of $x$ contains a maximal totally singular isotropic space. Thus we can write $V_{2}=U \oplus U^{\prime}$ where $U$ and $U^{\prime}$ are maximal complementary totally isotropic spaces, with $U$ the fixed space of $x$. Thus $x$ is trivial on $V_{2} / U$ as well and so $x$ is in the unipotent radical $Q$ of the stabilizer of $U$. Then $x$ corresponds to a full rank skew symmetric matrix in $Q$. Thus, the centralizer of $x$ is $Q \cdot \operatorname{Sp}(U)$ with $\operatorname{Sp}(U)$ acting isomorphically on both $U$ and $U^{\prime}$. Since $\operatorname{Sp}(U)$ is transitive on non-zero vectors of $U$, it is easy to see that given $v=u+u^{\prime}$, we can move $u, u^{\prime}$ into a nondegenerate $x$-invariant 4-space.

Thus we are reduced to $\mathrm{GO}_{4}(k)$ and $\mathrm{GO}_{6}(k)$. It is straightforward to check that in the first case there is a unique orbit, while in the second case there are two orbits (as can be seen for example by checking that over finite fields $\mathbb{F}_{q}$, there are two orbits, of lengths $\left.q^{3}\left(q^{2}-1\right), q^{2}(q-1)\right)$.

\section{Proposition 6.2.}

(a) Let $G=D_{4}(k) .3$, the extension by a graph automorphism, and $x \in G^{\circ}$ a long root element. Then $\left|G_{2}(k) \backslash G^{\circ} / C_{G^{\circ}}(x)\right|=5$ and $\left|G_{2}(k) \backslash G / C_{G}(x)\right|=3$.

(b) Let $G=E_{6}(k) .2$, the extension by a graph automorphism, and $x \in G^{\circ}$ a long root element. Then $\left|F_{4}(k) \backslash G / C_{G}(x)\right|=2$.

Proof. First consider $G=D_{4}(k) .3$, the extension by the graph automorphism $\sigma$ of order 3 . Let $B$ be a $\sigma$-stable Borel subgroup of $G^{\circ}$. Let $P$ be the normalizer in $G^{\circ}$ of a $\sigma$-stable root subgroup, a maximal parabolic subgroup of type $A_{1}^{3}$. Its derived subgroup $P^{\prime}$ is the centralizer of a long root element in $G^{\circ}$. In order to show that $G_{2}(k) \backslash G / P^{\prime}$ is finite, it suffices to see that $G_{2}(k)$ has finitely many orbits on the set of long root elements of $G^{\circ}$. We claim that there are precisely five orbits. Clearly, there's one orbit of long root elements (in $B$ ) centralized by $\sigma$; these are long root elements in $H=C_{G^{\circ}}(\sigma) \cong G_{2}(k)$ 
and thus have centralizer $Q^{\prime}$ in $H$, where $Q$ is a maximal parabolic subgroup of $H$. Furthermore, there are three orbits of long roots fused under $\sigma$, such that the product over any $\sigma$-orbit is a short root element in $H$. These have centralizer $U_{3} \cdot A_{1}(k)$, with $U_{3}$ unipotent of dimension 3. Finally, let $x \in U=R_{u}(B)$ be the product of a root element with support 2 (on the simple roots of $G^{\circ}$ with respect to $B$ ) with a commuting root element with support 3 . Then the centralizer of $u$ in $U \cap H$ has dimension 4 . The product $u u^{\sigma} u^{\sigma^{2}}$ is a unipotent element $\left(x_{a+b}(1) x_{2 a+b}(1)\right.$ with respect to the simple roots $a, b$ of $H$ ) with 4-dimensional unipotent centralizer in $H$. Thus $\operatorname{dim}\left(C_{H}(u)\right)=4$. (This is the element constructed in Example 4.4 when $p \neq 3$, respectively in Example 3.4 when $p=3$.)

For $p>0$, over the field with $q=p^{a}$ elements the corresponding $G_{2}(q)$-orbits in $D_{4}(q) .3$ have lengths $q^{6}-1, q^{2}\left(q^{6}-1\right)(3$ times $)$, and $q^{2}\left(q^{2}-1\right)\left(q^{6}-1\right)$, which adds up to the number of long root elements, so we've accounted for all orbits in this case. By arguing as in [7, Ex. 6.2] we conclude that the claim holds over any algebraically closed field. Note that since $\sigma$ permutes the three orbits of dimension 8, we obtain three orbits under $C_{G}(\sigma)$.

Now consider $G=E_{6}(k) .2$, the extension by the graph automorphism $\sigma$ of order 2 . The centralizer of a long root element in $G^{\circ}$ is the derived subgroup $P^{\prime}$ of a parabolic subgroup $P$ of $G^{\circ}$ of type $A_{5}$. The group $H=C_{G^{\circ}}(\sigma) \cong F_{4}(k)$ has one orbit on long root elements contained in $H$, so centralized by $\sigma$, with centralizer $Q^{\prime}$, where $Q$ is an end-node parabolic subgroup of $H$. Now let $x$ be a long root element not fixed by $\sigma$, corresponding to the root $\alpha=\sum_{i=1}^{6} a_{i} \alpha_{i}$, with $\alpha_{1}, \ldots, \alpha_{6}$ the simple roots of $E_{6}$ in the standard numbering and $\left(a_{1}, \ldots, a_{6}\right)=(1,1,1,2,2,1)$. This root subgroup is normalized by a maximal torus of $H$, and also centralized by all but three positive root subgroups in $H$. Moreover, $\alpha$ is centralized by a subgroup of type $W\left(A_{3}\right)$ in the Weyl group of $H$. Thus $C_{H}^{\circ}(x)=U \cdot A_{3}(k)$ with $U$ unipotent of dimension 15 , and the $H$-orbit of $x$ has dimension $22=\operatorname{dim} E_{6}(k)-\operatorname{dim} P^{\prime}$, so it is the dense orbit. Note that the $\sigma$-conjugate root element $x^{\sigma}$ (in the root subgroup corresponding to (112211)) has exactly the same connected centralizer in $H$, and for $p \neq 2, C_{H}\left(x x^{\sigma}\right)=U \cdot A_{3}(k) .2$ is an extension of $C_{H}^{\circ}(x)$ of degree 2 (see [14]). Since $W\left(F_{4}\right)$ contains an element interchanging the root subgroups of $x$ and $x^{\sigma}$, we deduce that $C_{H}(x)=U \cdot A_{3}(k)$ is connected for $p \neq 2$. A direct computation in $E_{6}(2)$ shows that $C_{H}(x)$ is also connected in characteristic 2.

In the finite group over $\mathbb{F}_{q}$, the lengths $\left(q^{4}+1\right)\left(q^{12}-1\right)$ and $q^{3}\left(q^{4}+1\right)\left(q^{12}-1\right)$ of these two orbits add up to the total number of long root elements. We conclude as in the previous case.

We now turn to the proof of Theorem 1.1 from the introduction. One step is given by the following result:

Lemma 6.3. Let $G$ be an almost simple algebraic group over an algebraically closed field, and let $C, D$ be $G^{\circ}$-classes. If every $\left(x_{1}, x_{2}\right) \in C \times D$ normalizes some Borel subgroup of $G^{\circ}$ then $C D$ is a finite union of $G^{\circ}$-conjugacy classes.

Proof. Let $\left(x_{1}, x_{2}\right) \in C \times D$ normalize the Borel subgroup $B$ of $G^{\circ}$. If $x_{1}$, say, is inner, then conjugates of $x_{1}$ inside $B$ have only finitely many distinct semisimple parts, so the possible products with $x_{2}$ have only finitely many quasi-semisimple classes in their 
closures, whence we get (i) by [7, Lemma 5.1] (note the argument shows that the number of possible quasi-semisimple classes is independent of the Borel subgroup).

So now let's assume that $C$ and $D$ are both outer. We claim that this cannot occur aside from the case that $C$ and $D$ are in distinct cosets of outer involutions (whence $\left.G=D_{4}(k) \cdot \mathfrak{S}_{3}\right)$.

Note that under the assumption that every pair in $C \times D$ normalizes a Borel subgroup, the conclusion holds if and only if it holds for $C^{\prime} \times D^{\prime}$ where $C^{\prime}$ and $D^{\prime}$ are the closed quasi-semisimple classes in $\bar{C}$ and $\bar{D}$ respectively. So it suffices to assume that $C$ and $D$ are both quasi-semisimple. Note that our condition is also true for powers of $C$ and $D$.

By choosing a parabolic subgroup that is invariant under the graph automorphism and such that it still induces a graph automorphism on its Levi subgroup, we can reduce to the cases $A_{1}(k)^{2}, A_{2}(k)$ or $A_{1}(k)^{3}$ (the latter in $D_{4}(k)$ ). It is straightforward in each of these cases to check that there is not always a common Borel subgroup unless possibly $C$ and $D$ are in distinct cosets of graph automorphisms of order 2. Moreover, in the latter case, if neither $C$ nor $D$ have order 2, then one sees (as in the proof of Proposition 7.4), there will not always be a common invariant Borel subgroup. So we may assume that $C$ consists of graph automorphisms of order 2. Similarly, if $D$ is not as given in Proposition 7.4(1), there will not always be a common invariant Borel subgroup. If $D$ does satisfy Proposition 7.4(1), then in fact there are only finitely many classes in $C D$.

We are now ready to prove Theorem 1.1, which is part of the following statement:

Theorem 6.4. Let $G$ be an almost simple algebraic group over an algebraically closed field, and let $C, D$ be $G^{\circ}$-classes in $G$. The following are equivalent:

(i) $C D$ is a finite union of $G^{\circ}$-conjugacy classes.

(ii) The closure of $C D$ contains a unique quasi-semisimple conjugacy class.

(iii) $C_{G^{\circ}}\left(x_{1}\right) \backslash G^{\circ} / C_{G^{\circ}}\left(x_{2}\right)$ is finite for all $\left(x_{1}, x_{2}\right) \in C \times D$.

(iv) $G^{\circ}$ has finitely many orbits on $C \times D$.

(v) $\left\langle x_{1}, x_{2}\right\rangle$ normalizes some Borel subgroup of $G^{\circ}$ for every $\left(x_{1}, x_{2}\right) \in C \times D$.

(vi) $C_{G^{\circ}}\left(x_{1}\right) C_{G^{\circ}}\left(x_{2}\right)$ is dense in $G^{\circ}$ for some $\left(x_{1}, x_{2}\right) \in C \times D$.

Moreover, these conditions imply, and if $G \neq D_{4} \cdot \mathfrak{S}_{3}$ are all equivalent to

(vii) $[C, D]$ is a finite union of $G^{\circ}$ conjugacy classes.

Proof. Statements (i) and (ii) are equivalent by Corollary 2.4 and clearly (iii) and (iv) are equivalent. Furthermore, (iii) implies (vi). By [7, Lemma 5.3] we have that (vi) implies (ii).

It is shown in Lemma 6.3 that (v) implies (i). The equivalence of (i) and (vii) is the statement of Theorem 5.2 .

Thus it remains to show that (i) implies (iii) and (v), which we do by going through the cases in Theorem 4.6. For the cases in (1), this is [7, Cor. 5.14]. The unipotent examples in (2) are those from Theorem 3.7 (2)-(5). Here, it is shown in Examples 3.2, 3.4, 3.5, and in [7, Ex. 6.6] that $\left\langle x_{1}, x_{2}^{g}\right\rangle$ is a unipotent subgroup and thus (v) holds. Furthermore, we have (iii) by Example 3.2 and Propositions 6.1 and 6.2.

Now let's turn to the non-unipotent examples in Theorem 4.6(e)-(k). For case (e), there is just one orbit of $G$ on $x_{1}^{G} \times x_{2}^{G}$ by [7, Ex. 7.2], and clearly we can find a pair in the normalizer of some Borel subgroup, whence (iii) and (v) hold. In case (f), we have just 
two orbits by Example 3.2. Since clearly the graph automorphism $\sigma$ cannot centralize all transvections in a $\sigma$-stable Borel subgroup of $G^{\circ}$, we get representatives of both orbits in the normalizer.

In case (g), by Example 4.2 there's again just a single orbit. In case (h), by Example 4.3 both elements always lie in the normalizer of some parabolic subgroup, and $x_{1}$ in its unipotent radical, so we get (v), while (iii) is in Proposition 6.1. Finally, in cases (i) and (j), by Example 4.4 there is a dense pair, which lies inside a Borel subgroup, and (iii) was shown in Proposition 6.2. If $G=D_{4} \cdot \mathfrak{S}_{3}$, we apply Proposition 7.4 and Lemma 7.5.

The same result holds for $G$-classes as well (but we can include (vii) without any restrictions as all the cosets of outer involutions are conjugate).

Note that all in cases we see from the above arguments that $G$ either has infinitely many orbits or at most 3 orbits on $C_{1} \times C_{2}$. This was shown in the connected case in [7] aside from two cases where a bound of 4 was given. We show that 3 is an upper bound in these cases as well in the next two results.

Proposition 6.5. Let $k$ be an algebraically closed field of characteristic 2. Let $G=$ $\operatorname{Sp}_{2 n}(k)=\operatorname{Sp}(V)$. Let $x \in G$ be an involution with $(x v, v)=0$ for all $v \in V$. Then $C_{G}(x)$ has at most 3 orbits on the nonzero vectors of $V$.

Proof. Note that $W:=[x, V]$ is a totally singular $m$-space for some $m \leq n$. Let $X=$ $[x, V]^{\perp}$. Let $P$ be the stabilizer of $W$. So $P=Q L$ is a maximal parabolic subgroup of $G$ with unipotent radical $Q$ and Levi subgroup $L \cong \mathrm{GL}(W) \times \operatorname{Sp}(X / W)$. Note that $x \in Z(Q)$ and so we see that $C_{G}(x)=Q J$ where $J \cong \operatorname{Sp}(W) \times \operatorname{Sp}(X / W)$. The result now follows easily by noting that $J$ acts transitively on the nonzero elements of $W, X / W$ and $V / X$ and that if $v \in X \backslash W$, then $Q v=v+W$ and if $v \in V \backslash X$, then $Q v=v+X$ (if $W \neq X$, the three orbits are $W \backslash\{0\}, X \backslash W$ and $V \backslash X$ while if $W=X$, there are two orbits).

Proposition 6.6. Let $k$ be an algebraically closed field of characteristic $p \neq 2$. Let $G=\mathrm{SO}_{n}(k)=\mathrm{SO}(V)$. Let $x \in G$ be a unipotent element with all Jordan blocks of size at most 2. Then $C=C_{G}(x)$ has at most 3 orbits on nondegenerate 1-spaces of $V$.

Proof. First suppose that $n$ is a multiple of 4 and all Jordan blocks of $x$ have size 2. Then $W:=[x, V]=C_{V}(x)$ is a maximal totally singular subspace and $C=Q \operatorname{Sp}(W)$ where $Q$ is the unipotent radical of the stabilizer of $W$. Then $C$ is transitive on all cosets $v+W$ with $v$ outside $W$ and $Q$ is transitive on all vectors in $v+W$ of a given norm, whence we see that $C$ has a single orbit on nondegenerate 1-spaces (this is also clear from the proof of Proposition 6.1). Thus, we see that $C$ has two orbits on nonzero singular vectors (they are either in $W$ or not).

In the general case, we can write $V=V_{0} \perp W$ where $V_{0}$ is a nondegenerate space with $x$ trivial on $V_{0}$ and all Jordan blocks of $x$ have size 2 on $W$. Let $v$ be a nonsingular vector in $V$ and write $v=v_{0}+w$ with $v_{0} \in V$ and $w \in W$. By the argument in the first paragraph, we can find $x$-invariant nondegenerate subspaces $V_{1} \subset V_{0}$ and $W_{1} \subset W$ with $\operatorname{dim} V_{1} \leq 2$ and $\operatorname{dim} W_{1} \leq 4$ and $c \in C$ with $c v \in V_{1}+W_{1}$. Thus, we may assume that $n \leq 6$ (and clearly $n \geq 4$ ). It is straightforward to check the result in these cases.

This completes the proof of Corollary 1.2 . 


\section{Cosets OF ORDER 2 IN $D_{4}(k) \cdot \mathfrak{S}_{3}$}

Here, we discuss the situation left open in conclusion $(\mathrm{k})$ of Theorem 4.6 ,

Let $k$ be an algebraically closed field of characteristic $p \geq 0$ and $G=D_{4}(k) \cdot \mathfrak{S}_{3}$ (of adjoint type), the extension of a simple algebraic group of type $D_{4}$ by the full group of graph automorphisms $\mathfrak{S}_{3}$. Let $x, y \in G$ be outer involutions in two different cosets of $G^{\circ}$. Moreover, we assume that $x$ and $y$ correspond to reflections in some projective 8-dimensional representation of $\left\langle G^{\circ}, x\right\rangle$, resp. $\left\langle G^{\circ}, y\right\rangle$. Note that $G^{\circ}=C_{G^{\circ}}(x) C_{G^{\circ}}(y)$ whence $G^{\circ}$ is transitive on such pairs. Set $z=x y$. Thus $z$ is a graph automorphism of $G^{\circ}$ of order 3 with centralizer $C_{G^{\circ}}(z) \cong G_{2}(k)$.

We start with some examples. In all of these $C$ denotes the $G^{\circ}$-class of $x$ and $D$ will be a class in the coset $G^{\circ} y$. For convenience we recall the outer unipotent conjugacy classes of $\mathrm{GO}_{8}(k)$, with $k$ algebraically closed of characteristic 2 (see [15, p.236] and [10, Tab. 10], and the unipotent classes of $G_{2}(k)$ (see [9, Tab. B, Tab. 1]).

TABLE 1. Outer unipotent classes of $\mathrm{GO}_{8}(k), \operatorname{char}(k)=2$

\begin{tabular}{|l|cccc|}
\hline$x$ & $C(x) / R_{u}(C(x))$ & $\operatorname{dim} R_{u}(C(x))$ & $\operatorname{dim} C(x)$ & $A(u)$ \\
\hline $2.1^{6}$ & $C_{3}$ & 0 & 21 & 1 \\
$2^{3} .1^{2}$ & $A_{1}^{2}$ & 7 & 13 & 1 \\
$4.1^{4}$ & $A_{1}^{2}$ & 5 & 11 & $Z_{2}$ \\
$3^{2} .2$ & $A_{1}$ & 6 & 9 & 1 \\
$4.2^{2}$ & $A_{1}$ & 6 & 9 & 1 \\
$4.2^{2}$ & 1 & 7 & 7 & 1 \\
$6.1^{2}$ & $T_{1}$ & 4 & 5 & $Z_{2}$ \\
8 & 1 & 3 & 3 & 1 \\
\hline
\end{tabular}

TABLE 2. Unipotent classes of $G_{2}(k), \operatorname{char}(k) \neq 3$

\begin{tabular}{|l|cccc|rr|}
\hline$x$ & $C(x) / R_{u}(C(x))$ & $\operatorname{dim} R_{u}(C(x))$ & $\operatorname{dim} C(x)$ & $A(u)$ & \multicolumn{2}{|c|}{ in $D_{4}(k)$} \\
& & & & & $(p>3)$ & $(p=2)$ \\
\hline 1 & $G_{2}$ & 0 & 14 & 1 & $1^{8}$ & $1^{8}$ \\
$A_{1}$ & $A_{1}$ & 5 & 8 & 1 & $2^{2} .1^{4}$ & $2^{2} \cdot 1^{4}$ \\
$\tilde{A}_{1}$ & $A_{1}$ & 3 & 6 & 1 & $3.2^{2} .1$ & $2^{4}$ \\
$G_{2}\left(a_{1}\right)$ & 1 & 4 & 4 & $\mathfrak{S}_{3}$ & $3^{2} .1^{2}$ & $3^{2} .1^{2}$ \\
$G_{2}$ & 1 & 2 & 2 & $Z_{(2, p)}$ & 7.1 & 6.2 \\
\hline
\end{tabular}

Example 7.1. Let $D$ consist of elements conjugate to $y s=s y$ where $s \in C_{G^{\circ}}(y)$ is a semisimple element with at most 2 non-trivial eigenvalues. First assume that $s$ has a non-trivial eigenvalue $a$ of order prime to 6 . Note that $\operatorname{dim} C=7$ and that $\operatorname{dim} D=$ 17. Also note that we can arrange that $x y$ is conjugate to $z t$ where $t \in C_{G^{\circ}}(z)$ with $t$ semisimple and of order prime to 6 . Thus $\operatorname{dim} C_{G^{\circ}}(z t)=\operatorname{dim} C_{G_{2}(k)}\left(t^{3}\right) \leq 4$ and it follows 
TABLE 3. Unipotent classes of $G_{2}(k), \operatorname{char}(k)=3$

\begin{tabular}{|l|cccc|r|}
\hline$x$ & $C(x) / R_{u}(C(x))$ & $\operatorname{dim} R_{u}(C(x))$ & $\operatorname{dim} C(x)$ & $A(u)$ & in $D_{4}(k)$ \\
\hline 1 & $G_{2}$ & 0 & 14 & 1 & $1^{8}$ \\
$A_{1}$ & $A_{1}$ & 5 & 8 & 1 & $2^{2} .1^{4}$ \\
$\tilde{A}_{1}$ & $A_{1}$ & 5 & 8 & 1 & $3.2^{2} .1$ \\
$\tilde{A}_{1}^{(3)}$ & 1 & 6 & 6 & 1 & $3.2^{2} .1$ \\
$G_{2}\left(a_{1}\right)$ & 1 & 4 & 4 & $Z_{2}$ & $3^{2} .1^{2}$ \\
$G_{2}$ & 1 & 2 & 2 & $Z_{3}$ & 7.1 \\
\hline
\end{tabular}

that $\operatorname{dim}(x y)^{G^{\circ}} \geq 24$, whence we have equality. Thus, $C D=(x y)^{G^{\circ}}$ (since the right hand side is closed). Indeed the same argument shows that for any $\left(x^{\prime}, y^{\prime}\right) \in C \times D$, $\operatorname{dim}\left(C_{G^{\circ}}\left(x^{\prime}\right) \cap C_{G^{\circ}}\left(y^{\prime}\right)\right) \leq \operatorname{dim} C_{G^{\circ}}(x y)=4$, so $G^{\circ}=C_{G^{\circ}}(x) C_{G^{\circ}}(y)$. If $a \in k^{\times}$is arbitrary, the centralizer will only get bigger whence we still have the same factorization.

Example 7.2. Let $p \neq 2$ and $D$ consist of elements conjugate to $y u=u y$ where $u \in$ $C_{G^{\circ}}(y)$ is unipotent with Jordan block lengths $2^{2} .1^{4}$ or $3.1^{5}$.

First consider the case that $u$ is a long root element. Note that every $G^{\circ}$-orbit in $C \times D$ contains a representative $(x, y u)$ where $u$ is a long root element commuting with $y$ (since $G^{\circ}$ is transitive on $\left.x^{G^{\circ}} \times y^{G^{\circ}}\right)$. By Proposition 6.2, $C_{G^{\circ}}(z)$ has five orbits on long root elements in $G$. It is easy to see that only 2 of these orbits are contained in $C_{G}(y)$. Thus, $G^{\circ}$ has exactly 2 orbits on $C \times D$ (depending upon whether $u$ centralizes $z$ or not).

There is a subgroup $A_{1}(k)^{4}$ of $G^{\circ}$ normalized by $x, y$. We may assume that $y$ permutes the last two copies and $x$ permutes the middle two copies (so both centralize the first copy). If we take $u=\left(a_{1}, a_{2}, 1,1\right)$ where $a_{i}$ are nontrivial unipotent elements of $A_{1}(k)$, then $u$ has a single Jordan block of size 3 but $z u$ is conjugate to $z v=v z$ where $v$ has two Jordan blocks of size 3 (and two trivial Jordan blocks). It follows that $v$ lies in the conjugacy class $G_{2}\left(a_{1}\right)$ in $C_{G^{\circ}}(z)$. Thus, $\operatorname{dim}(z v)^{G^{\circ}}=24$ and so $G^{\circ}$ has a dense orbit on $C \times D$ (consisting of those pairs whose product is in the class of $z v$ ).

Example 7.3. Let $p=2$ and $D$ consist of 2-elements with Jordan block lengths $2^{3} \cdot 1^{2}$, $4.1^{4}$ or $3^{2} .2$ in the natural 8-dimensional representation of $\mathrm{GO}_{8}(k)$. An explicit calculation in the permutation representation of $\mathrm{SO}_{8}^{+}(2) \cdot \mathfrak{S}_{3}$ of degree 3510 (as maximal subgroup of $F i_{22}$ ) shows that the respective products contain elements of the form $z u$, with $z=x y$ a graph automorphism of order 3 and $u \in C_{G^{\circ}}(z) \cong G_{2}(k)$ unipotent in the $G_{2}(k)$-class $\tilde{A}_{1}, G_{2}\left(a_{1}\right), G_{2}$ respectively (see Table 2). Thus the corresponding classes $E=[z u]$ have dimensions 22,24, 26 respectively. As $\operatorname{dim} C+\operatorname{dim} D$ equals $7+15,7+17,7+19$ in the three cases, we conclude by Lemma 3.1 that $E$ is dense in $C D$ and that $C D$ consists of finitely many $G^{\circ}$ classes.

We now show that the above are the only examples:

Proposition 7.4. Let $C, D$ be $G^{\circ}$-orbits in $x G^{\circ}$ and $y G^{\circ}$. Then $C D$ is a finite union of $G^{\circ}$-orbits if and only if (after reordering if necessary) $C$ consists of involutions conjugate (in $G$ ) to $x$ and one of 
(1) $D$ consists of elements conjugate to $y t=t y$ where $t \in G^{\circ}$ is a semisimple element with at most 2 non-trivial eigenvalues;

(2) $p \neq 2, D$ consists of elements conjugate to $y u=u y$ where $u$ is unipotent with Jordan block lengths $2^{2} .1^{4}$ or $3.1^{5}$; or

(3) $p=2$ and $D$ consists of 2 -elements with Jordan block lengths $2^{3} .1^{2}, 4.1^{4}$ or $3^{2} .2$ in the natural 8-dimensional representation of $\mathrm{GO}_{8}(k)$.

Proof. We have already seen in Examples 7.17 .3 that all cases (1)-(3) really occur. So we need to show that these are the only possibilities with $C D$ containing only finitely many $G^{\circ}$-orbits. So assume that $C D$ consists of finitely many $G^{\circ}$-orbits or equivalently that all quasi-semisimple elements in the closure of $C D$ are conjugate.

First consider the case that $p \neq 2$ and $C$ and $D$ are both involutions but neither are reflections. Note that this prescribes $C$ and $D$ uniquely. Thus, for any pair of involutions $s, t \in C_{G^{\circ}}(z),(x s, y t) \in C \times D$ and $x s y t=z(s t)$. As $s, t$ run over all such involutions, st intersects infinitely many $G_{2}(k)$ classes (since we can take $s, t$ to be involutions in $G_{2}(k)$ ), whence $C D$ consists of infinitely many classes.

If $p=2$ and $C$ and $D$ both consist of 2-elements, then every unipotent class is represented in $\operatorname{Aut}\left(D_{4}(2)\right)$ and one checks directly using GAP that the answer is as stated in (3) of the assertion.

So next assume that $D$ does not consist of involutions and that $C$ and $D$ are both quasisemisimple classes. Then we choose $\left(x^{\prime}, y^{\prime}\right) \in C \times D$ which normalize a parabolic subgroup $P=Q L$ with $P$ the normalizer of a long root subgroup, $L$ is a Levi subgroup and $Q$ the unipotent radical of $P$. Note that $L=T A_{1}(k)^{3}$ where $T$ is a central 1-dimensional torus of $L$. We can assume that $x$ permutes the first two copies of $A_{1}(k)$ and $y$ the last two.

Since $C$ and $D$ consist of quasi-semisimple elements, we can choose $x^{\prime}, y^{\prime}$ actually normalizing $L$. It is straightforward to see that (aside from the cases allowed in the conclusion) $(C \cap L)(D \cap L)$ intersects infinitely many classes of $L . \mathfrak{S}_{3}$ whence $C D$ contains infinitely many $G^{\circ}$-orbits by Proposition 3.6 .

Thus, we may assume that all quasi-semisimple elements in the closure of $x^{\prime}$ are conjugate to $x$ and that the quasi-semisimple elements in the closure of $y^{\prime}$ are as given in (1) or (2). Moreover, at least one of $x^{\prime}, y^{\prime}$ is not quasi-semisimple.

Case 1. $x^{\prime}$ is quasi-semisimple and $y$ does not lie in the closure of $y^{\prime}$.

By passing to closures, we may assume that $y^{\prime}=y_{1} y=y y_{1}$ where $y_{1}=u t$ with $t$ semisimple having precisely two nontrivial eigenvalues and $u$ is a long root element. Note that such a class has a representative in $L$ and one easily checks that there are infinitely many classes represented there.

Case 2. $x^{\prime}$ is quasi-semisimple and $y$ lies in the closure of $y^{\prime}$.

In this case, we can assume that $p \neq 2$ (because of the remarks above about pairs of 2-elements). By passing to closures (and noting that we may assume the unipotent part of $y^{\prime}$ is not a long root element nor has just one single Jordan block of size 3), we may assume that $y^{\prime}=y u$ where $u$ has a single Jordan block of size 3 and two Jordan blocks of size 2. Again, we can see such an element in $L$ and obtain a contradiction.

Case 3. Neither $x^{\prime}$ nor $y^{\prime}$ are quasi-semisimple.

Passing to closures, we may assume that the unipotent parts of $x^{\prime}$ and $y^{\prime}$ are long root elements and again we can see such elements in $L$ and derive a contradiction. 
By symmetry, we have covered all cases and proved the result.

Lemma 7.5. In the situation of Proposition 7.4. $G^{\circ}$ acts transitively on $C \times D$ in case (1); it has 2 orbits in both situations of case (2); and there are 2, 3, respectively 2 orbits in case (3). Moreover, every $(c, d) \in C \times D$ normalizes a Borel subgroup of $G^{\circ}$.

Proof. The case (1) was already argued in Example 7.1, and similarly for the first pair of classes in case (2), in Example 7.2, If $u$ has Jordan blocks of lengths $3.1^{5}$ in case (2), we need to count orbits of $G_{2}(k)$ on the class of $u$ in $\mathrm{SO}_{7}(k)$.

Note that if $u \in \mathrm{SO}_{7}(k)$ is such a unipotent element, it has a unique 1-dimensional invariant (singular) subspace (namely the image of $(u-1)^{2}$ ) and then $u$ lies in the unipotent radical of the stabilizer $P_{1}$ of that subspace (corresponding to nonsingular vectors in the unipotent radical).

Note that $G_{2}(k)$ acts transitively on singular 1-spaces (this is well known, but note that in the 7-dimensional representation, the stabilizer of a highest weight space is a maximal parabolic subgroup $P$ of codimension 5 , thus $G_{2} / P$ is a 5-dimensional projective variety contained in $\mathrm{SO}_{7}(k) / P_{1}$ which is also projective of dimension 5 , so they are equal). So we can just count orbits of $P$ on the unipotent radical $Q$ of $P_{1}$. Now $Q$ is 5-dimensional and abelian and $P_{1} / Q$ acts on this as $T \mathrm{SO}_{5}(k)$, and $P /(Q \cap P)=T A_{1} U_{3}$ acts on this so that $Q=2 / 1 / 2$ as a module for $P$. Because of the torus it is enough to count orbits on nondegenerate 1-spaces, whence it is easy to see that $P^{\prime}$ has two such orbits acting on $Q$ (namely those spaces in the orthogonal complement of the 2 and those outside).

Finally, in case (3), first assume that $D$ contains unipotent elements with Jordan type $2^{3} \cdot 1^{2}$. As we saw in Example 7.3, one orbit consists of pairs $(c, d)$ such that $c d$ lies in class $\tilde{A}_{1}$ of a subgroup $G_{2}(k)$, with connected centralizer of dimension 6 . A second orbit is given by pairs $(c, d)$ with product in class $A_{1}$ in $G_{2}(k)$, and connected centralizer of dimension 8. Adding up the lengths of these two orbits over $\mathbb{F}_{2^{f}}$ shows that there no other orbits.

Next, assume that $D$ contains unipotent elements with Jordan type $4.1^{4}$. Any $d \in D$ can be written as $y w$ where $y$ is a reflection and $w$ is a long root element in $G^{\circ}$ (not centralizing $y$ for sure). This is not unique, but given any $(c, d) \in C \times D$, we can conjugate and assume $c=x$ and $d=y w$ as above. Now $G_{2}(k)$ centralizes $x$ and $y$ and so the number of orbits of $G$ on $C \times D$ is at most the number of orbits of $G_{2}(k)$ on the set of long root elements $w$ such that $y w$ has Jordan type $4.1^{4}$.

Since $G_{2}(k)$ has 5 orbits on long root elements and at least two of them do not have the property that $y w$ has order 4 (namely the ones in $G_{2}(k)$ and also the ones in $\operatorname{Sp}_{6}(k)$ ), there are at most 3 orbits. In fact, we claim that there are 3 orbits. Two of the orbits are interchanged by $y$ and we can see such a $w$ in $A_{1}(k) \times A_{1}(k)$ where $y$ is interchanging the 2 factors and $w$ is trivial in one of the factors. Clearly $y w$ has order 4 and has a 5-dimensional fixed spaces, so $y w$ has the correct Jordan type. Since $y$ interchanges those two orbits, we see that there are at least 2 orbits. However, neither of these is the dense orbit (e.g., they have the same dimension) and so there must be a third orbit (alternatively, one can compute over $\mathbb{F}_{2}$ ).

Finally consider the case that $D$ consists of unipotent elements with Jordan type $3^{2} .2$. 
Computing over $\mathbb{F}_{2}$, we see that $C D$ contains the conjugacy classes of elements $z u=u z$ where $z$ is a graph automorphism of order 3 with centralizer $G_{2}(k)$ and $u$ is either a regular unipotent element of $G_{2}(k)$ or is in the conjugacy class $G_{2}\left(a_{1}\right)$.

First consider a pair $(c, d) \in C \times D$ with $c d=z u=u z$ where $u$ is a regular unipotent element in $G_{2}(k)$. It follows that $J$, the centralizer of $\langle c, d\rangle$ is contained in the centralizer of $z u$ which has connected component a 2-dimensional unipotent group and has two components. Since $\operatorname{dim} C+\operatorname{dim} D=26$, it follows that $\operatorname{dim} J \geq 2$, whence $J^{\circ}$ is a 2dimensional unipotent group. Moreover, this must correspond to the dense orbit of $G^{\circ}$ and so it is unique. Computing such triples over $\mathbb{F}_{2}$, we see that there are two orbits each of size $\left|G^{\circ}(2)\right| / 4 / 2$. It follows by Lang's theorem that $\left[J: J^{\circ}\right]=2$ and so there is a single $G^{\circ}$-orbit splitting into two $G(q)$-orbits each of size $(1 / 2)\left|G^{\circ}(q)\right| / q^{2}$.

It follows by counting that the dimension of the complement of the dense orbit in $C \times D$ is 24 and moreover, there is at most 1 orbit of that dimension. Let $H$ be the centralizer of a $(c, d) \in C \times D$ with product $z u=u z$ where $u \in G_{2}\left(a_{1}\right)$. The centralizer $H$ of $z u$ in $G^{\circ}$ has connected component a 4-dimensional unipotent group with $H / H^{\circ} \cong \mathfrak{S}_{3}$ (see Table 2). We see that over $\mathbb{F}_{2}$ this orbit breaks up into three orbits of sizes $e\left|G^{\circ}(2)\right| / 2^{4}$ withe $e=1 / 2,1 / 3$ and $1 / 6$. It follows that $H$ is the centralizer of $\langle c, d\rangle$ and this orbit breaks up into 3 orbits over $\mathbb{F}_{q}$ of sizes $e\left|G^{\circ}(q)\right| / q^{4}$ with $e=1 / 2,1 / 3$ and $1 / 6$. Thus, the number of $\mathbb{F}_{q}$ points in this orbit of the algebraic group is $\left|G^{\circ}(q)\right| / q^{4}$.

Since $|C(q)| D(q)|=| G^{\circ}(q)\left|/ q^{4}+\right| G^{\circ}(q) \mid / q^{2}$, it follows that there are no further orbits and so $G^{\circ}$ has exactly 2 orbits on $C \times D$.

The last assertion follows by noting that in the examples we produce $(c, d) \in C \times D$ in the dense orbit which normalize a Borel subgroup. Since normalizing a Borel subgroup is a closed condition (as $G / B$ is a projective variety), this implies the result.

We next consider commutators of $G^{\circ}$-orbits in our situation. Here, we have the following curious situation:

Example 7.6. As above let $x, y \in G=D_{4}(k) . \mathfrak{S}_{3}$ be quasi-central in distinct cosets of order 2 modulo $H:=G^{\circ}$. Then for any $g \in H$ we have

$$
[x, y g]=x(y g)^{-1} x y g=x g^{-1}(y x y) g \in x^{H} t^{H}=(x t)^{H}
$$

where $t=y x y$ is a quasi-central element (reflection) in the third such coset. So letting $C=$ $x^{H}$ and $D=y H$, we see that $[C, D]$ is a single $H$-orbit (namely the graph automorphisms of order 3 with centralizer $\left.G_{2}(k)\right)$. In particular, $G$ certainly need not have finitely many orbits on $C \times D$. Hence, the analogue of Theorem 6.4(vii) fails in this situation.

Proposition 7.7. Let $G=D_{4}(k) \cdot \mathfrak{S}_{3}$ and $C, D$ two $G^{\circ}$-orbits in distinct cosets of $G^{\circ}$ of order 2. Then $[C, D]$ is the union of finitely many $G^{\circ}$-orbits if and only if (up to order) $C$ consists of reflections.

Proof. We have seen in Example 7.6 that if $C$ consists of reflections and $D$ is any class, then $[C, D]=z^{G^{\circ}}$ where $z$ is a graph automorphism of order 3 with centralizer $G_{2}(k)$. Thus, by taking closures we may assume that one of the following holds:

(1) $C$ and $D$ are both quasi-semisimple;

(2) $C$ and $D$ are both reflections times long root elements; or

(3) $C$ is quasi-semisimple and $D$ is as in (2). 
We can see all these cases in $A_{1}^{4}$ and get a contradiction (assuming that neither $C$ nor $D$ consists of reflections).

Here is another corollary (which could be proved more directly):

Corollary 7.8. Let $G$ be an almost simple algebraic group. Suppose that $C_{1}, C_{2}, C_{3}$ are nontrivial $G^{\circ}$-orbits. Then $C_{1} C_{2} C_{3}$ consists of infinitely many $G^{\circ}$-orbits unless (up to order) $C_{1}$ and $C_{2}$ are reflections in different cosets modulo $G^{\circ}$ and $C_{3}$ consists of long root elements.

Proof. Observe that for $C_{1}$ and $C_{2}$ reflections in different cosets and $C_{3}$ long root elements, $C_{1} C_{2}=D:=z^{G^{\circ}}$ and so indeed $C_{1} C_{2} C_{3}=D C_{3}$ is a finite union of classes by Theorem 4.6.

In any case other than $D_{4}(k) \cdot \mathfrak{S}_{3}$ with an outer automorphism, two of the $C_{i}$ must either be inner or outer and so already $C_{i} C_{j}$ consists of infinitely many inner classes again by Theorem 4.6 (and by working in the normalizer of a torus, infinitely many classes times $C_{k}$ gives infinitely many classes).

If $G$ is connected, by passing to closures, we may assume that each $C_{i}$ is either semisimple or unipotent. We know that two semisimple elements do not work and so at least two of the classes must be unipotent. By taking closures, they can be taken to be root elements (and so for roots of distinct lengths). So we reduce to the rank 2 case, i.e. $B_{2}(k)$ (characteristic 2) or $G_{2}(k)$ (characteristic 3) by [7]. But then the product of the two unipotent classes contains a regular unipotent element and that is not one of our examples.

\section{INFINITE FIELDS}

Let's also note the following easy consequence of our results in [7]:

Corollary 8.1. If $G$ is a simple compact (real) Lie group and $C$ and $D$ are non-central conjugacy classes of $G$, then $C D$ is an infinite union of classes.

Proof. It follows easily from the fact that $G$ is Zariski dense in $\bar{G}$, the complexification of $G$, that any class is dense in its closure $\bar{C}$ in $\bar{G}$. Now if $C D$ is a finite union of classes, then taking closures shows that $\bar{C} \bar{D}$ is a finite union of $\bar{G}$-classes as well, and as $C$ and $D$ are semisimple classes, by [7, Thm. 1.1] this cannot happen.

For the disconnected case, we would get only the semisimple cases (e.g. in type $A$ ). The previous result extends to infinite fields with $G(K)$ an anisotropic simple group (i.e., containing no nontrivial unipotent elements).

The same ideas give the following result.

Corollary 8.2. Let $K$ be an infinite field and $G(K)$ some form of a simple algebraic group $G$ over $K$. Let $C \subset G(K)$ be a non-central conjugacy class. Then $C C$ is an infinite union of classes.

Proof. Let $\bar{C}$ denote the Zariski closure of $C$. Then the semisimple part of elements in $\bar{C}$ is unique, since in any rational faithful representation, elements in $\bar{C}$ have the same characteristic polynomial as those in $C$, as $G(K)$ is Zariski dense in $G$ (see e.g. [16, Cor. 13.3.9]). Thus, if $C C$ consists of finitely many classes, so does $\bar{C} \bar{C}$, which is not possible by [7, Thm. 1.1]. 


\section{REFERENCES}

[1] M. Aschbacher, G. Seitz, Involutions in Chevalley groups over fields of even order. Nagoya Math. J. 63 (1976), 1-91.

[2] F. Digne, J. Michel, Groupes réductifs non connexes. Ann. Sci. École Norm. Sup. (4) 27 (1994), $345-406$.

[3] D. Gorenstein, R. Lyons, R. Solomon, The Classification of the Finite Simple Groups. Number 3. Mathematical Surveys and Monographs, American Mathematical Society, Providence, RI, 1998.

[4] R. M. Guralnick, A note on pairs of matrices with rank one commutator. Linear and Multilinear Algebra 8 (1979/80), 97-99.

[5] R. M. Guralnick, Intersections of conjugacy classes and subgroups of algebraic groups. Proc. Amer. Math. Soc. 135 (2007), 689-693.

[6] R. M. Guralnick, G. Malle, Variations on the Baer-Suzuki theorem. In preparation.

[7] R. M. Guralnick, G. Malle, P. H. Tiep, Products of conjugacy classes in finite and algebraic simple groups. Advances Math. 234 (2013), 618-652.

[8] N. Katz, Rigid Local Systems. Annals of Mathematics Studies, 139. Princeton University Press, Princeton, NJ, 1996.

[9] R. Lawther, Jordan block sizes of unipotent elements in exceptional algebraic groups. Comm. Algebra 23 (1995), 4125-4156.

[10] G. Malle, Generalized Deligne-Lusztig characters. J. Algebra 159 (1993), 64-97.

[11] G. Malle, Green functions for groups of types $E_{6}$ and $F_{4}$ in characteristic 2. Comm. Algebra 21 (1993), 747-798.

[12] G. Prasad, Weakly-split spherical Tits systems in quasi-reductive groups. Amer. J. Math. (to appear), arXiv: 1103.5970 .

[13] R. W. Richardson, On orbits of algebraic groups and Lie groups. Bull. Austral. Math. Soc. 25 (1982), 1-28.

[14] T. ShoJI, The conjugacy classes of Chevalley groups of type $\left(F_{4}\right)$ over finite fields of characteristic $p \neq 2$. J. Fac. Sci. Univ. Tokyo Sect. IA Math. 21 (1974), 1-17.

[15] N. Spaltenstein, Classes Unipotentes et Sous-Groupes de Borel. Lecture Notes in Math., 946. Springer-Verlag, Berlin, 1982.

[16] T. A. Springer, Linear Algebraic Groups. Second edition. Progress in Mathematics, 9. Birkhäuser Boston, Boston, MA, 1998.

[17] R. Steinberg, Endomorphisms of Linear Algebraic Groups. Memoirs Amer. Math. Soc., 80, American Mathematical Society, Providence, RI, 1968.

Department of Mathematics, University of Southern California, Los Angeles, CA 90089-2532, USA

E-mail address: guralnic@usc.edu

FB Mathematik, TU Kaiserslautern, Postfach 3049, 67653 Kaiserslautern, Germany

E-mail address: malle@mathematik.uni-kl.de 